\title{
Oscillatory Control of Factors Determining Multipotency and Fate in Mouse Neural Progenitors.
}

\section{$\operatorname{AUTHOR}(S)$ :}

Imayoshi, Itaru; Isomura, Akihiro; Harima, Yukiko; Kawaguchi, Kyogo; Kori, Hiroshi; Miyachi, Hitoshi; Fujiwara, Takahiro; Ishidate, Fumiyoshi; Kageyama, Ryoichiro

\section{CITATION:}

Imayoshi, Itaru ...[et al]. Oscillatory Control of Factors Determining Multipotency and Fate in Mouse Neural Progenitors.. Science 2013, 342(6163): 1203-1208

\section{ISSUE DATE:}

2013-10-31

URL:

http://hdl.handle.net/2433/179383

\section{RIGHT:}

(C) 2013 American Association for the Advancement of Science.; This is not the published version. Please cite only the published version.; この 論文は出版社版でありません。引用の際には出版社版をご確認ご利用 ください。 


\title{
Title: Oscillatory control of determination factors for multipotency versus fate choice in mouse neural progenitors
}

Authors: Itaru Imayoshi ${ }^{1,2,3,4} \dagger^{*}$, Akihiro Isomura ${ }^{1,5} \dagger$, Yukiko Harima ${ }^{1,5}$, Kyogo Kawaguchi ${ }^{6}$, Hiroshi Kori $^{5,7}$, Hitoshi Miyachi ${ }^{1}$, Takahiro Fujiwara ${ }^{3}$, Fumiyoshi Ishidate ${ }^{3}$, and Ryoichiro Kageyama $^{1,3,5 *}$

\begin{abstract}
Affiliations:
${ }^{1}$ Institute for Virus Research, Kyoto University, Shogoin-Kawahara, Sakyo-ku, Kyoto 606-8507, Japan.

2 The Hakubi Center, Kyoto University, Kyoto 606-8302, Japan.

${ }^{3}$ World Premier International Research Initiative-Institute for Integrated Cell-Material Sciences (WPI-iCeMS), Kyoto University, Kyoto 606-8501, Japan.

${ }^{4}$ Japan Science and Technology Agency, Precursory Research for Embryonic Science and Technology (PRESTO), 4-1-8 Honcho, Kawaguchi, Saitama, 332-0012, Japan.

${ }^{5}$ Japan Science and Technology Agency, Core Research for Evolutional Science and Technology (CREST), 4-1-8 Honcho, Kawaguchi, Saitama, 332-0012, Japan.

${ }^{6}$ Department of Physics, Graduate School of Science, The University of Tokyo, Tokyo 113-0033, Japan.

${ }^{7}$ Department of Information Sciences, Ochanomizu University, Tokyo 112-8610, Japan.

*Correspondence to: Ryoichiro Kageyama, Institute for Virus Research, Kyoto University, Shogoin-Kawahara, Sakyo-ku, Kyoto 606-8507, Japan. E-mail: rkageyam@virus.kyoto-u.ac.jp ; Itaru Imayoshi, The Hakubi Center, Institute for Virus Research, Kyoto University, ShogoinKawahara, Sakyo-ku, Kyoto 606-8507, Japan. E-mail: iimayosh@ virus.kyoto-u.ac.jp

$\dagger$ These authors contributed equally to this work.
\end{abstract}

\begin{abstract}
The basic-helix-loop-helix transcription factors Ascl1/Mash1, Hes1, and Olig2 regulate fate choice of neurons, astrocytes, and oligodendrocytes, respectively. These same factors are coexpressed by neural progenitor cells. Here, we found by time-lapse imaging that these factors are expressed in an oscillatory manner by mouse neural progenitor cells. In each differentiation lineage, one of the factors becomes dominant. We used optogenetics to control expression of Ascl1, and found that although sustained Ascl1 expression promotes neuronal fate determination, oscillatory Ascl1 expression maintains proliferating neural progenitor cells. Thus, the multipotent state correlates with oscillatory expression of several fate-determination factors, whereas the differentiated state correlates with sustained expression of a single factor.
\end{abstract}

\section{Word limit includes text + references + figure and table legends}


Main Text: Analyses of populations of mouse hematopoietic progenitors suggest that general fluctuations in the transcriptome affect lineage choice (1). On the other hand, analyses of individual cells do not show an effect of transcriptome fluctuation on lineage choice (2). Fate choice could be a gradual process based on transcriptome-wide fluctuations or discrete processes through different subpopulation states, To address these issues, we study the expression patterns of fate determination factors in individual mouse cells in the multipotent state and during fate choice processes.

In the developing murine nervous system, neural progenitor cells (NPCs) proliferate (reproducing themselves) and also differentiate into three cell types, neurons, oligodendrocytes, and astrocytes (thus demonstrating multipotency). Transcription factors that regulate the proliferation of NPCs and the differentiation of each cell type include basic helix-loop-helix (bHLH) transcription factors $(3,4)$. For example, proneural bHLH genes such as Ascl1/Mash1 and Neurogenin2 (Ngn2) promote neuronal fate determination and suppress astrocytic gene expression (5-7). The bHLH gene Olig 2 regulates oligodendrocyte specification, while the bHLH genes Hes 1 and Hes 5 maintain NPCs by repressing proneural gene expression (8-12). Ascl1 and Olig2 also regulate oligodendrocyte and motor neuron development, respectively (810,13-18). One model suggests that Hes genes, expressed by NPCs, repress expression of other bHLH genes; down-regulation of Hes genes allows up-regulation of proneural genes or Olig2 and subsequent differentiation into neurons or oligodendrocytes (4). However, Ascll is expressed by dividing NPCs and postmitotic neurons, and Ascll up-regulates the expression of genes involved in cell cycle progression of NPCs as well as in cell cycle exit and neuronal differentiation $(19,20)$. Furthermore, Hes 1 promotes the maintenance of NPCs and astrocyte differentiation $(11,12,21,22)$. Olig2 is also involved in NPC proliferation (23). Thus, these bHLH genes exert contradictory functions.

Hes 1 expression oscillates with a period of approximately 2-3 hours in many cell types including NPCs $(24,25)$. Ngn2 expression also oscillates in NPCs probably because it is periodically repressed by Hes 1 oscillation. In differentiating neurons, which lack Hes 1 expression, Ngn2 expression is steady (25). Thus, the pattern of bHLH gene expression differs between NPCs and neurons, although our previous time-lapse imaging study only monitored mRNA production (25). As transcription and translation can be dissociated (26), we here study protein expression at the single cell level. We found that Hes1, Ascl1, and Olig2 protein expression oscillates in NPCs.

\section{Variable levels of bHLH transcription factor expression in NPCs}

Ascl1, Hes1, and Olig2 are expressed by NPCs in the ventral telencephalon, which generate neurons, astrocytes, and oligodendrocytes during perinatal stages of mouse development (1418,27,28). The expression levels of these transcription factors were variable from cell to cell (Fig. 1A-H). Many cells were positive for all three bHLH factors (Hes1, Ascl1, and Olig2), while others expressed only two (Fig. 1E-H). By contrast, differentiating neurons, oligodendrocytes, and astrocytes expressed only one of them at later stages (i.e., Asc11, Olig2, and Hes1, respectively)(18,22,29). To examine the expression of these bHLH factors, we generated transgenic mice carrying reporters in which fluorescent (Venus or mCherry) or firefly luciferase (Luc2) cDNA was inserted in frame into the 5 ' region of each bHLH gene in BAC clones so that a bHLH factor fused with either Venus, mCherry, or Luc2 was expressed (table S1, fig. S1A-F). We also generated knock-in mice in which Venus was inserted in frame into the 5' region of the Hes 1 gene for Hes1 imaging (table S1, fig. S2A), those in which Venus or luciferase (Eluc) was 
inserted in frame into the $5^{\prime}$ region of the Hes 5 gene for Hes5 imaging (table S1, fig. S2B,C), and Sox 2 reporter mice expressing a Luc2-Sox 2 fusion protein (table S1, fig. S1G). The reporter expression in these mice was similar to endogenous expression (fig. S3). Reporter expression also correlated well with endogenous protein expression in NPCs (fig. S4). The brain structures and the NPC competency of these reporter mice including homozygous Venus-Hes1 fusion knock-in mice were apparently normal (fig. S5).

We used time-lapse imaging of brain slices from the ventral telencephalon of reporter mice and found that Hes 1 and Ascl1 expression oscillate in NPCs (Fig. 1I-K, movie S1).

\section{Oscillatory expression of bHLH factors in NPCs}

We next prepared NPCs from the ventral telencephalon of perinatal reporter mice. We used acutely dissociated NPCs and those maintained in vitro for at least ten generations in the presence of EGF and bFGF (NS cells)(30,31). Both cell types exhibited similar expression oscillations in the progenitor state and during cell fate choice. These NPCs express Ascl1, Olig2, and Hes1 at variable levels (fig. S6) and generate GABAergic neurons, oligodendrocytes, and astrocytes (fig. S7, (14)). Their competency to generate neurons and oligodendrocytes was significantly reduced in the absence of Ascl1 and Olig2, respectively (fig. S7G,H',I,K). These NPCs also showed a reduced ability to generate astrocytes in the absence of Hes1 (fig. S7F',J). Compared to the bHLH factors, the NPC-specific factor Sox2 (32) was expressed at a relatively constant level (fig. S6A,E,J).

We used time-lapse imaging to analyze expression patterns of bHLH factors. Hes 1 protein expression oscillated in NPCs (average period $149.9 \pm 12.3 \mathrm{~min}$ )(Fig. 2A,D,G,H, fig. S8, movie S2). Hes5 expression in NPCs oscillated in phase with Hes1 expression (figs. S9 and S10). Ascl1 expression oscillated in NPCs (average period $175.4 \pm 29.5 \mathrm{~min}$ )(Fig. 2B,E,G,I, fig. S11, movie S3). The Ascl1 protein level was up-regulated during S/G2 in some cells ( 30\%, fig. S11C,D); after cell division both daughter cells showed equal amounts of Ascl1, and expression oscillations resumed in cells that remained undifferentiated (fig. S11D). Olig2 protein expression also oscillated, but more slowly (average period $375.5 \pm 105.8 \mathrm{~min}$ ) than Hes1 and Ascl1 protein oscillations (Fig. 2C,F,G, fig. S12, movie S4). The average expression levels of these factors are different between $\mathrm{G} 1$ and S/G2/M (fig. S13), although their expression oscillated throughout the cell cycle. By contrast, two other factors characteristic of NPCs were expressed steadily: Sox 2 and Nestin (figs. S13C and S14, movie S5). Hes1 and Ascl1 expression are inversely correlated (fig. S15A,D, (33)); Hes1 represses Ascl1 expression by binding to the Ascl1 promoter (34). Oscillating Hes1 would periodically repress Ascl1 expression, thereby driving oscillations in Ascl1 expression. Indeed, oscillatory Ascl1 expression was lost in the absence of Hes1 (fig. S11E,F). Expression of Olig2, on the other hand, changes independently of Ascl1 and Hes1 (fig. S15B,C,E,F).

We next examined whether the oscillations in bHLH factor expression levels create a bias in differentiation competency. We separated NPCs of the fluorescent reporter mice into Hes1-high, Hes1-low, Ascl1-high, Ascl1-low, Olig2-high, and Olig2-low fractions (fig. S16A-C). All cell fractions kept in NS media returned to original diverse levels of Hes1, Ascl1, and Olig2 expression within 2 days, suggesting that diverse levels represent different phases of expression oscillation (fig. S16D-L). All cells generated neurospheres at similar efficiencies (fig. S16M-R). After sorting, each cell population was cultured in a differentiation medium. Hes1-high and Hes1-low cells preferentially differentiated into astrocytes and neurons, respectively (fig. S17A,B). Ascl1-high (and Olig-2 low) and Olig2-high (and Ascl1-low) cells preferentially 
differentiated into neurons and oligodendrocytes, respectively (fig. S17A,C-G). These results suggest that the different expression levels of the bHLH factors bias the fate choice of NPCs. However, such transient high expression is neither required nor sufficient for cell fate determination: NPCs with high expression of any of Asc11, Olig2, and Hes1 were all able to differentiate into any of the three cell types (fig. S17).

\section{Sustained expression of bHLH factors during cell fate choice}

We next examined how the expression of these bHLH factors changes during cell fate choice. During neuronal differentiation, Ascl1 protein accumulated after cell division (Fig. 3A-D), in contrast to oscillatory expression in NPCs, and 6-8 hours later, the early neuronal marker Doublecortin (DCX) was expressed (Fig. 3A,C-D'). Ascl1 expression continued to be upregulated in many cells $(76.7 \%$, Fig. 3A-C) but not in others $(23.3 \%$, Fig. 3D) after DCX expression was initiated, raising the possibility that the minimum requirement for neuronal differentiation is accumulation of Ascl1 over 6 to 8 hours during G1.

In acute dissociation culture from the ventral telencephalon, many NPCs underwent asymmetric cell division in which one daughter cell remained undifferentiated while the other differentiated into a neuron. In these NPCs, Ascl1 expression was up-regulated (at least two-fold compared to the average) before cell division and seemed to be equally distributed in both daughter cells (Fig. 3E). In the daughter neuron, Ascl1 expression accumulated after cell division, while it resumed oscillating in the daughter NPC (Fig. 3E). Before neurogenic cell division (one or both daughter cells underwent neuronal differentiation), Ascl1 expression was transiently upregulated (at least two-fold compared to the average) in many cases: $~ 90 \%$ of their mother cells exhibited such transient up-regulation of Ascl1 before cell division (fig. S18B). However, 30\% of mother cells did so when they produced two daughter NPCs (figs. S11C,D and S18B). Thus, the transient up-regulation of Ascl1 before cell division is not decisive, but merely lends a bias toward neuronal fate choice.

In those NPCs whose daughter cells underwent neuronal differentiation, Hes1 expression was repressed before cell division (Fig. 3E', fig. S18A,E,F) but not when both cells remained NPCs (fig. S18A,C,D). The suppression of Hes1 expression was maintained in daughter neurons (Fig. 3E', fig. S18E,F), while Hes1 oscillation resumed in daughter NPCs (Fig. $\left.3 E^{\prime}\right)$. Thus, it is likely that transient down-regulation of Hes1 expression and the concomitant upregulation of Ascl1 before cell division directs NPCs toward neuronal fate choice, and that sustained expression of Ascl1 after cell division irreversibly determines neuronal fate. Downregulation of Hes1 could be caused by fluctuations of the expression levels of Notch intracellular domain (NICD), an active form of Notch signaling (35)(fig. S19). Indeed, when stable NICD expression was induced from the Rosa26 locus in NPCs, both Hes1 and Hes5 expression oscillated sustainably, and there was no down-regulation of these factors (figs. S20 and S21). By contrast, in the presence of a $\gamma$-secretase inhibitor, which inhibits Notch signaling activity, Ascl1 and Olig2 expression was up-regulated to stable expression (fig. S22).

During astrocyte differentiation, Hes1 protein expression still oscillated but at high average and trough levels (Fig. 4A,B, fig. S23B,C). 12-24 hours later, expression of the astrocyte marker GFAP began (fig. S23A). Ascl1 and Olig2 expression became undetectable within 10 hours during astrocyte differentiation (fig. S23D-G). During oligodendrocyte differentiation, Olig2 protein expression oscillated, but at high trough levels (Fig. 4C,D, fig. S24A,C,E). A few days after induction of oligodendrocyte differentiation, Olig2 expression was down-regulated, and expression of the mature oligodendrocyte marker 2',3'-Cyclic-nucleotide-3'- 
phosphodiesterase (CNPase)(fig. S25) was up-regulated (Fig. 4E, fig. S24B,D,G). During this period, Ascl1 and Hes1 expression were down-regulated (fig. S24H-J). Thus, bHLH fate determination factors are coexpressed in an oscillatory manner in NPCs, but as the cell fate choice becomes established, one factor accumulates and the other two are lost. Although oscillatory expression of multiple fate determination factors underlies the multipotent state of NPCs, this oscillatory pattern gives way to stable and dominant expression of one factor during cellular differentiation.

\section{Light-induced control of expression pattern}

To demonstrate the functional importance of oscillatory or sustained expression patterns, we adopted the optogenetic gene expression system using the Neurospora crassa photoreceptor Vivid that was fused with Ga14 DNA-binding domain and p65 activation domain (GAVPO)(36). The codon usage was optimized for mammalian cells to increase expression efficiency, and the target mRNA was destabilized by introducing the 3'-untranslated region of mouse Ascll mRNA (fig. S26A). With this system, we can induce gene expression comparable to endogenous levels (fig. S26D-G). Repeated exposure of blue light with 3-hour intervals generated oscillatory expression with a 3-hour period, while repeated exposure with 30-min intervals generated sustained expression at both cell population (Fig. 5A) and single cell levels (Fig. 5B,C, movie S6).

Because Ascll is known to promote the cell cycle progression of NPCs and their cell cycle exit and neuronal differentiation (20), we asked whether these contradictory functions of Ascl1 are regulated by different expression patterns. We introduced the Ascl1-inducible system into Ascll-null NS cells, which produce no neurons (fig. S7) and proliferate more slowly than wild-type NS cells (20). Ascll-null NS cells have a low proportion (4-5\%; $\mathrm{PH}^{+}$) of dividing cells (Fig. 5E), while wild-type NS cells have 14-15\% $\mathrm{PH}^{+}$dividing cells (see Fig S29C). However, light-induced oscillatory expression of Ascl1 increased the proportion of dividing cells in the Ascl1-null NS cell population to $\sim 10 \% \mathrm{PH}^{+}$(Fig. 5E-G), suggesting that Ascl1 oscillation enhanced NPC proliferation. These NS cells did not differentiate into neurons ( $\beta$ III-tubulin ${ }^{+}$) even after 3 days (Fig. 5D). The period of this oscillation is important, because a 6-hour period did not affect NPC proliferation (fig. S27). By contrast, light-induced sustained expression of Ascl1 enhanced neuronal differentiation ( $\beta$ III-tubulin ${ }^{+}$) of Ascl1-null NS cells (Fig. 5H-K). A higher level of sustained Ascl1 expression increased the efficiency of neuronal differentiation (Compare Fig. 5H versus fig. S28E). However, oscillatory Ascl1 expression did not induce neuronal differentiation even at a higher amplitude (fig. S28A-C) but increased the number of proliferating NPCs (fig. S28D). By contrast, sustained Ascl1 expression at similar levels increased neuronal differentiation (fig. S28E). These results indicate that distinct expression patterns, but not the levels, of Ascl1 are important for a choice between proliferation and differentiation.

We used the Ascl1-inducible system in wild-type NS cells where endogenous Ascl1 expression oscillates (fig. S29). Oscillatory light stimulation with 3-hour intervals did not affect the neurogenesis or proliferation of these cells (figs. S29A-I and S30A-D). By contrast, sustained Ascl1 expression for 72 hours increased neuronal formation even in the presence of bFGF and EGF, a condition that inhibits neurogenesis (figs. S29J-Q and S30E). At least 6-to-8 hours of sustained Ascl1 expression was required for generation of neurons (Fig. 5L-P), agreeing with the above notion about the minimal requirement for neuronal differentiation. This requirement for elapsed time suggests that only NS cells that caught at early G1 could be redirected by light- 
induced expression of Ascl1 into neuronal development. The Ascl1-inducible system was also introduced into the dorsal telencephalon, which normally expresses very low levels of Ascl1. The oscillatory expression of Ascl1 did not induce neuronal differentiation but did maintain Nestin ${ }^{+}$ NPCs in the ventricular zone, while the sustained expression of Ascl1 increased the number of $\beta$ III-tubulin ${ }^{+}$neurons that migrated out of the ventricular zone (fig. S31). Thus, manipulation of Ascl1 gene expression can impose a choice favoring proliferation or differentiation according to whether the Ascl1 expression is oscillatory or sustained.

\section{Discussion}

Our data suggest that multipotency is a state of multiple oscillating neurogenic and gliogenic determination factors, and that cell fate choice is a process of sustained expression of a single factor. This switching may be induced by the fluctuations of Notch signaling (Supplementary Text). The detailed mechanism by which the oscillatory and sustained Ascl1 expression differentially regulates downstream gene expression remains to be determined. It was reported that the proneural factor Ngn2 is differentially phosphorylated between NPCs and neurons and controls the expression of its target genes differently depending on its phosphorylation status (37). We speculate that the oscillatory and sustained expression of proneural factors could be involved in different posttranscriptional modulation that is responsible for target gene selectivity. We also demonstrated that the light-switchable gene expression system offers a new way to control the proliferation and differentiation of stem cells by changing the light exposure pattern rather than using different growth factors or chemicals, showing its applicability to the regeneration technology.

\section{References and Notes:}

1. H.H. Chang, M. Hemberg, M. Barahona, D.E. Ingber, S. Huang, Nature 453, 544-547 (2008).

2. C. Pina et al., Nat. Cell Biol. 14, 287-294 (2012).

3. N. Bertrand, D.S. Castro, F. Guillemot, Nat. Rev. Neurosci. 3, 517-530 (2002).

4. S.E. Ross, M.E. Greenberg, C.D. Stiles, Neuron 39, 13-25 (2003).

5. K. Tomita, K. Moriyoshi, S. Nakanishi, F. Guillemot, R. Kageyama, EMBO J. 19, 5460-5472 (2000).

6. Y. Sun et al., Cell 104, 365-376 (2001).

7. N. Nieto, S. Schuurmans, O. Britz, F. Guillemot, Neuron 29, 401-413 (2001).

8. Q.R. Lu et al., Cell 109, 75-86 (2002).

9. Q. Zhou, D.J. Anderson, Cell 109, 61-73 (2002).

10. H. Takebayashi et al., Curr. Biol. 12, 1157-1163 (2002).

11. T. Ohtsuka et al., EMBO J. 18, 2196-2207 (1999).

12. Y. Nakamura et al., J. Neurosci. 20, 283-293 (2000).

13. R. Mizuguchi et al., Neuron 31, 757-771 (2001).

14. C.M. Parras et al., EMBO J. 23, $4495-4505$ (2004).

15. S. Gokhan et al., J. Neurosci. 25, 8311-8321 (2005).

16. C.M. Parras et al., J Neurosci. 27, 4233-4242 (2007).

17. E.J. Kim, J. Battsite, Y. Nakagawa, J.E. Johnson, Mol. Cell. Neurosci. 38, 595-606 (2008).

18. M.A. Petryniak, G.B. Potter, D.H. Rowitch, J.L. Rubenstein, Neuron 55, 417-433 (2007).

19. E. Pastrana, L.-C. Cheng, F. Doetsch, Proc. Natl. Acad. Sci. USA 106, 6387-6392 (2009).

20. D.S. Castro et al., Genes Dev. 25, 930-945 (2011). 
21. T. Ohtsuka, M. Sakamoto, F. Guillemot, R. Kageyama, J. Biol. Chem. 276, 30467-30474 (2001).

22. Y. Wu, E.M. Levine M.S. Rao, Dev Dyn, 226, 675-689 (2003).

23. K.L. Ligon et al., Neuron 53, 503-517 (2007).

24. H. Hirata et al., Science 298, 840-843 (2002).

25. H. Shimojo, T. Ohtsuka, R. Kageyama, Neuron 58, 52-64 (2008).

26. R. Lu et al., Nature 462, 358-362 (2009).

27. S.W. Levison, J.E. Goldman, J. Neurosci. Res. 48, 83-94 (1997).

28. C.A.G. Marshall, J.E. Goldman, J. Neurosci. 22, 9821-9830 (2002).

29. S. Fukuda, T. Kondo, H. Takebayashi, T. Taga, Cell Death Differ. 11, 196-202 (2004).

30. L. Conti et al., PLoS Biol. 3, e283 (2005).

31. S.M. Pollard, L. Conti, Y. Sun, D. Goffredo, A. Smith, Cereb. Cortex 16, i112-i120 (2006).

32. L.H. Pevny, S.K. Nicolis, Int. J. Biochem. Cell Biol. 42, 421-424 (2010).

33. J.H. Baek., J. Hatakeyama, S. Sakamoto, T. Ohtsuka, R. Kageyama, Development 133, $2467-$ 2476 (2006).

34. H. Chen et al., Proc. Natl. Acad. Sci. USA 94, 5355-5360 (1997).

35. E.H. Schroeter, J.A. Kisslinger, R. Kopan, Nature 393, 382-386 (1998).

36. X. Wang, X. Chen, Y. Yang, Nat. Meth. 9, 266-269 (2012).

37. C. Hindley et al., Development 139, 1718-1723 (2012).

Acknowledgments: We are grateful to François Guillemot, Jane Johnson, Hirohide Takebayashi, Kazuhiro Ikenaka, Doug Melton, Atsushi Miyawaki, Asako Sakaue-Sawano, Qiang Lu, and Yi Yang for reagents, Satsuki Kitano, Masayuki Sakamoto, Hiromi Shimojo, and Center for Meso-Bio Single-Molecule Imaging (CeMI), WPI-iCeMS, Kyoto University for technical help. This work was supported by Core Research for Evolutional Science and Technology (R.K.,H.K.), Grant-in-Aid for Scientific Research on Innovative Areas (MEXT 22123002)(R.K.), Scientific Research (A)(JSPS 24240049)(R.K.), and Young Scientists (A)(JSPS 24680035)(I.I.), and Takeda Foundation (R.K.). I.I. and R.K. designed the project and wrote the manuscript. A.I. developed the light-induced gene expression system. I.I., A.I., Y.H., and H.M. performed experiments, and T.F. and F.I. conducted fluorescent imaging analyses. K.K. and H.K. performed computer simulation. A patent application, "Control of stem cells", on the control of proliferation and differentiation of stem cells, is in preparation by Kyoto University. 


\section{FIGURES}

Fig. 1. Variable expression levels of bHLH factors in NPCs of the ventral telencephalon. (A-H) The expression of Hes1, Ascl1, and Olig2 in the ventral telencephalon at perinatal stages was examined by immunohistochemistry. The boxed region in (D) is enlarged in (E-H). Many cells were positive for all three bHLH factors (Hes1, Ascl1 and Olig2, arrowheads), while others were mostly positive for two of them. (I-K) Bioluminescence imaging and quantification of Luc2Hes $1(\mathbf{I}, \mathbf{J})$ and Luc2-Asc11 (K) expression in slice cultures of the ventral telencephalon of reporter mice. Scale bars, $50 \mu \mathrm{m}(\mathbf{A}-\mathbf{D}, \mathbf{I})$.

Fig. 2. Oscillatory expression of bHLH factors in self-renewing NPCs derived from the ventral telencephalon. (A-F) Bioluminescence images and quantification of Luc2-Hes1 (A,D), Luc2Ascl1 (B,E), and Luc2-Olig2 (C,F) reporter expression. (G-I) The distribution of the oscillation periods of Hes1, Ascl1, and Olig2 derived from bioluminescence measurements $(n>25$ NPCs for each factor).

Fig. 3. Sustained Ascl1 expression in differentiating neurons. Bioluminescence images of Luc2Asc11 (A,B,C,D,E) and Luc2-Hes1 (E') expression were quantified. (A,C,C',D,D') Ascl1 expression accumulated in differentiating neurons (A, green line; $\mathbf{C , D}$, orange double asterisks). The early neuronal marker Doublecortin (Dcx) was monitored by DCX-DsRed. Transient upregulation of Ascl1 expression occurred before cell division (C,D, magenta asterisks). (B) Temporal trajectories of Luc2-Ascl1 in NS cells just after NPC or neurogenic division at time $=$ 0 (mean in solid line, and standard errors in colored, $\mathrm{n}>19$ for each division). Division-mode effect, $p=0.0022$; interaction between division-mode and time, $p<0.0001$, repeated measures ANOVA. (E) This acutely dissociated NPC underwent asymmetric cell division. Ascl1 was equally distributed in both daughter cells after the first cell division. Ascl1 expression accumulated in a daughter neuron (green) but resumed oscillating in a daughter NPC (red), which underwent the second division. (E') Hes1 expression was repressed before asymmetric cell division (asterisks). The suppression of Hes1 expression was maintained in a daughter neuron (blue line), whereas Hes1 oscillation resumed in a daughter NPC (red line).

Fig. 4. Expression dynamics of bHLH factors during gliogenesis. (A,B) Bioluminescence imaging (A) and quantification (B) of Luc2-Hes1 expression during astrocyte differentiation. Astrocyte specification was induced at time $=0$ by LIF and BMP4. (C-E) Bioluminescence imaging $(\mathbf{C})$ and quantification (D) of Luc2-Olig2 expression and quantification of pCNP-Venus (E) in a single cell during oligodendrocyte differentiation, which was induced by T3 at time $=0$.

Fig. 5. Light-induced oscillatory/sustained expression of Ascl1 in NPCs. (A-C) Time-lapse imaging $(\mathbf{C})$ and quantification $(\mathbf{A}, \mathbf{B})$ of gene expression at cell population $(\mathbf{A})$ and single cell levels $(\mathbf{B}, \mathbf{C})$ induced by blue light. (D-K) According to the schedule of light exposure (fig. S26B; light intensity: $1.11 \mu \mathrm{mol} / \mathrm{m}^{2} / \mathrm{s}$ ), oscillatory (D-G) and sustained (H-K) Ascl1 expression was induced in Ascll-null NPCs, which were cultured in the presence of bFGF and EGF, a condition that inhibits neurogenesis. Oscillatory Ascl1 expression induced virtually no $\beta$ IIItubulin $^{+}$neuron formation (D) but significantly increased the proportion of dividing cells ( $\mathrm{PH}^{+}$), compared to the control (Ub-luc2) (E-G). Sustained Ascl1 expression significantly increased 
$\beta$ III-tubulin ${ }^{+}$neuron formation, compared to the control (Ub-luc2) (H,J,K). ${ }^{*} p<0.05, * * p<0.01$; two-tailed Student's $t$-test. (L-P) According to the schedule of light exposure (fig. S26C), sustained Ascl1 expression was induced in NPCs for indicated time lengths, and neuronal formation was examined. ${ }^{*} p<0.05, * * p<0.01, * * * p<0.001$, one-way ANOVA followed by Tukey post hoc test. Scale bars, $12.5 \mu \mathrm{m}(\mathbf{F}, \mathbf{G}, \mathbf{J}, \mathbf{K}) ; 12.5 \mu \mathrm{m}$ (M-P).

\section{Supplementary Materials:}

Materials and Methods

Supplementary Text

Figures S1-S31

Table S1

Legends to Movies S1-S6

References (38-55) 

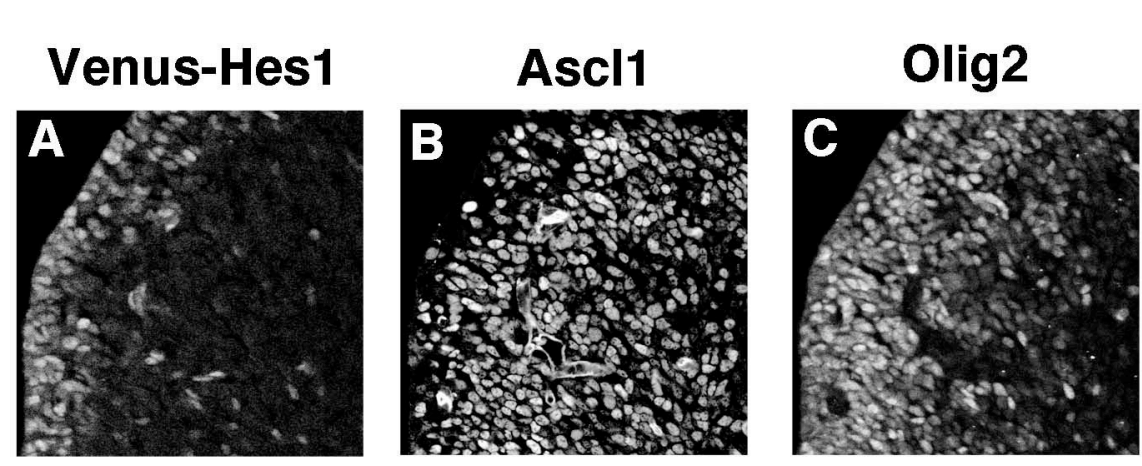

Venus-Hes1
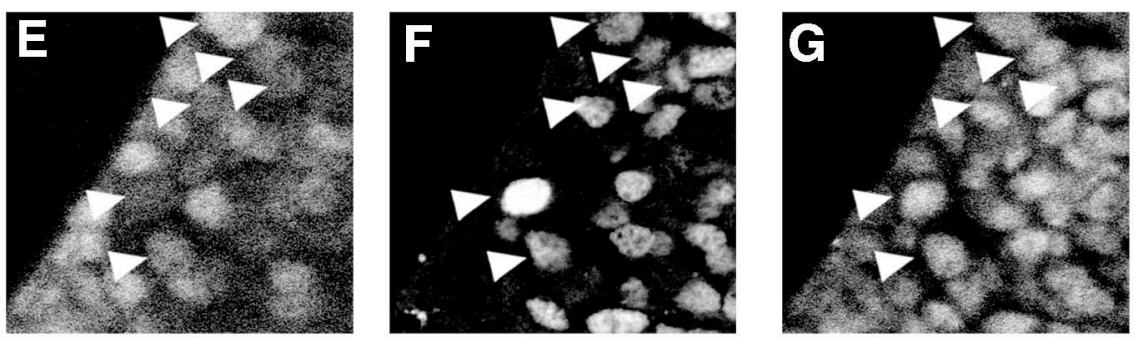

\section{Ascl1 Olig2}
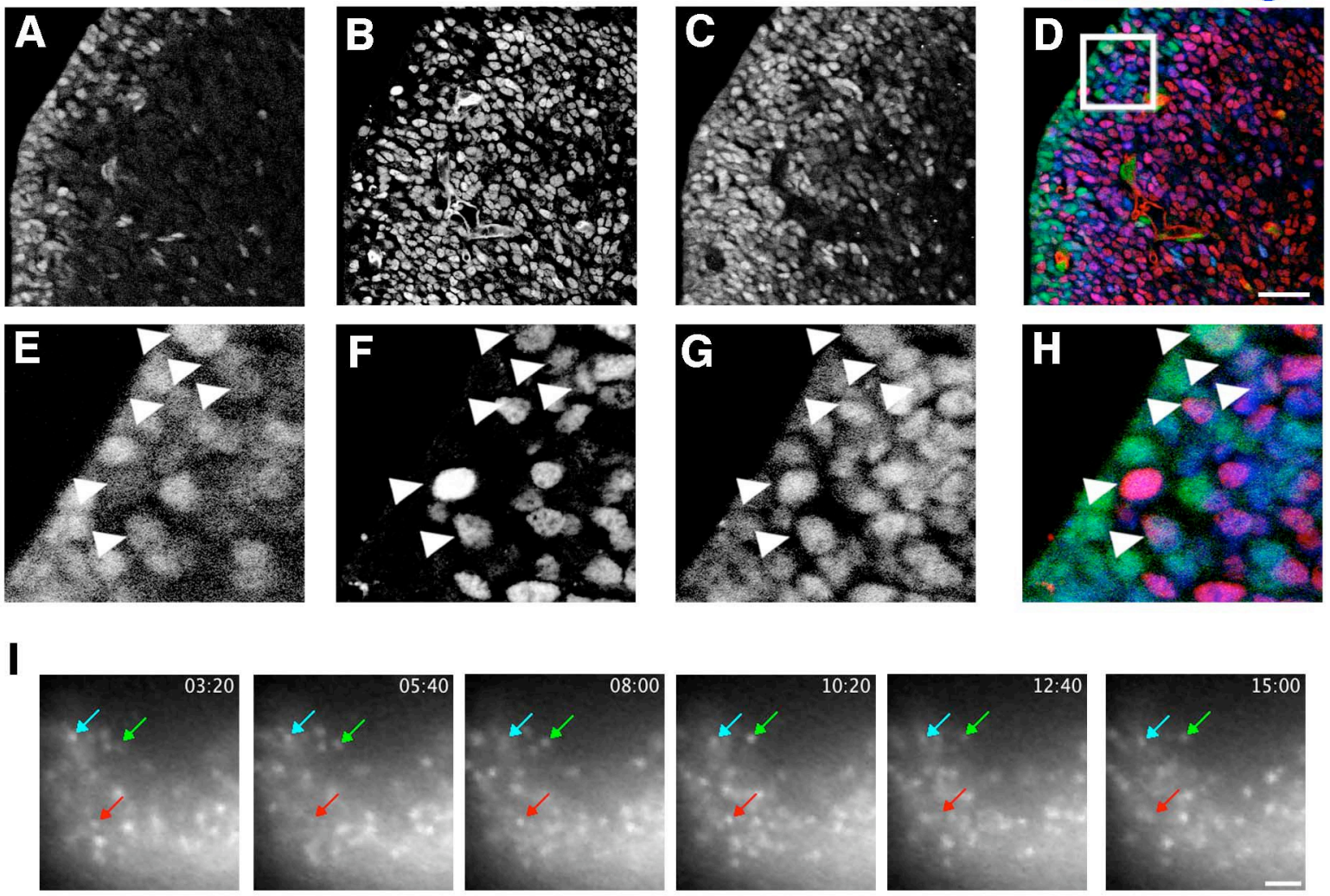

J

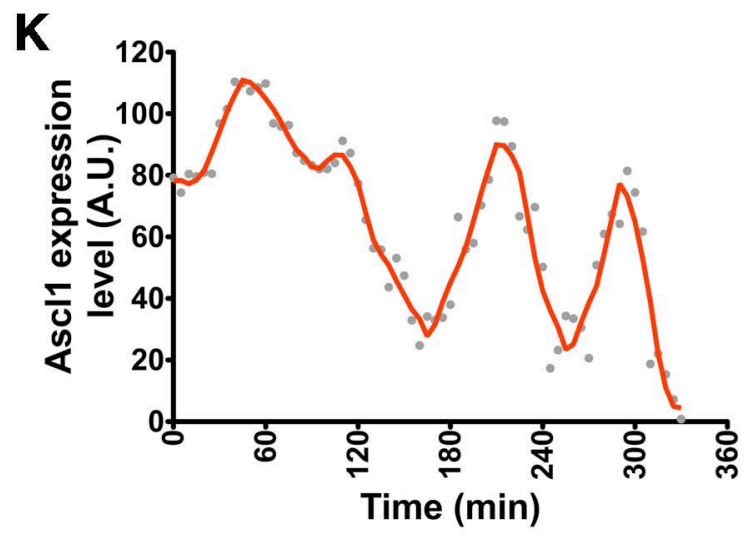

Time (min)

Figure 1 

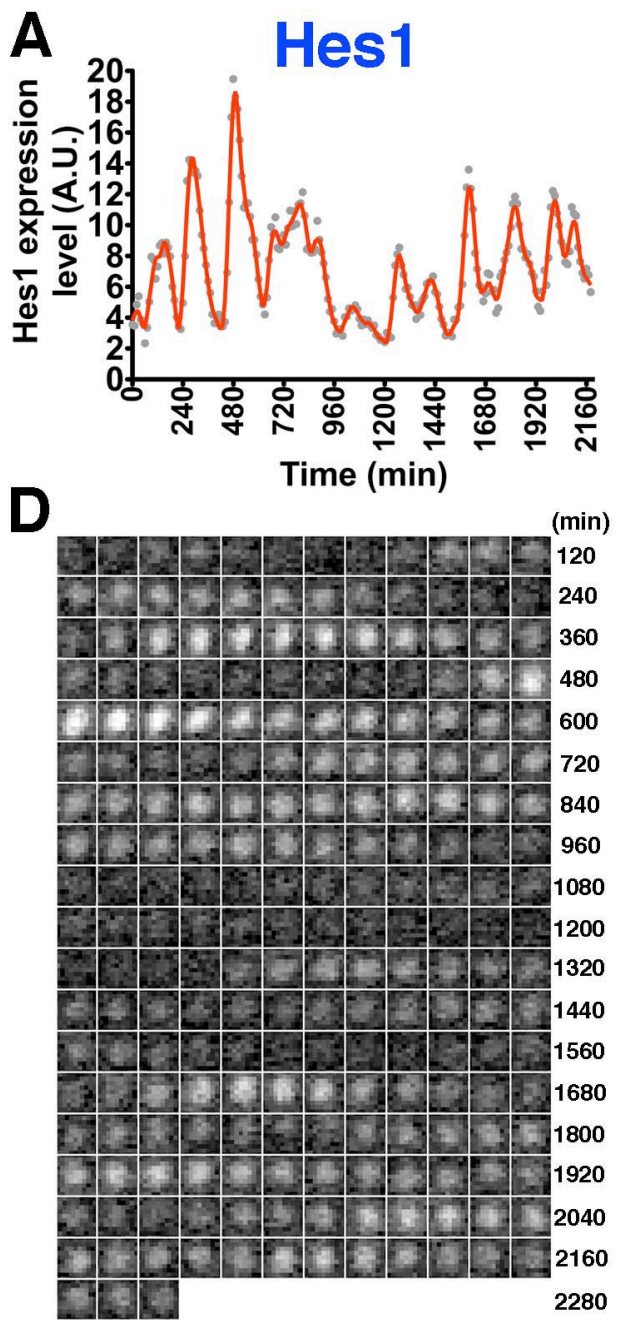
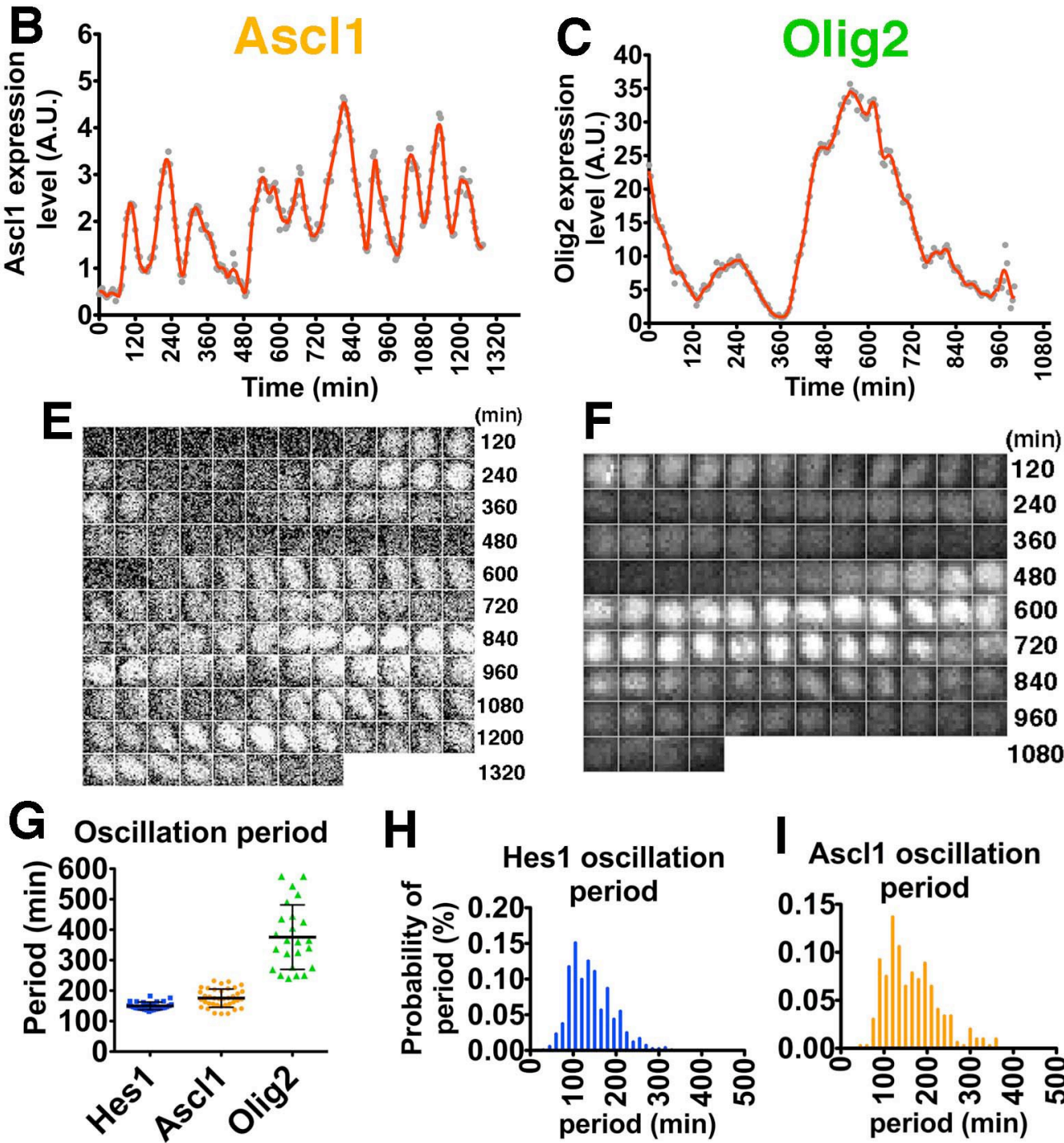

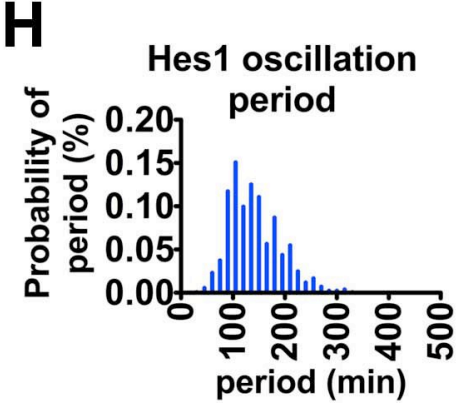

Ascl1 oscillation

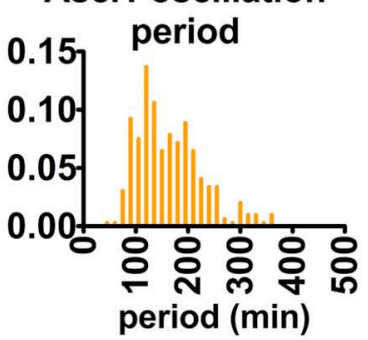

Figure 2 

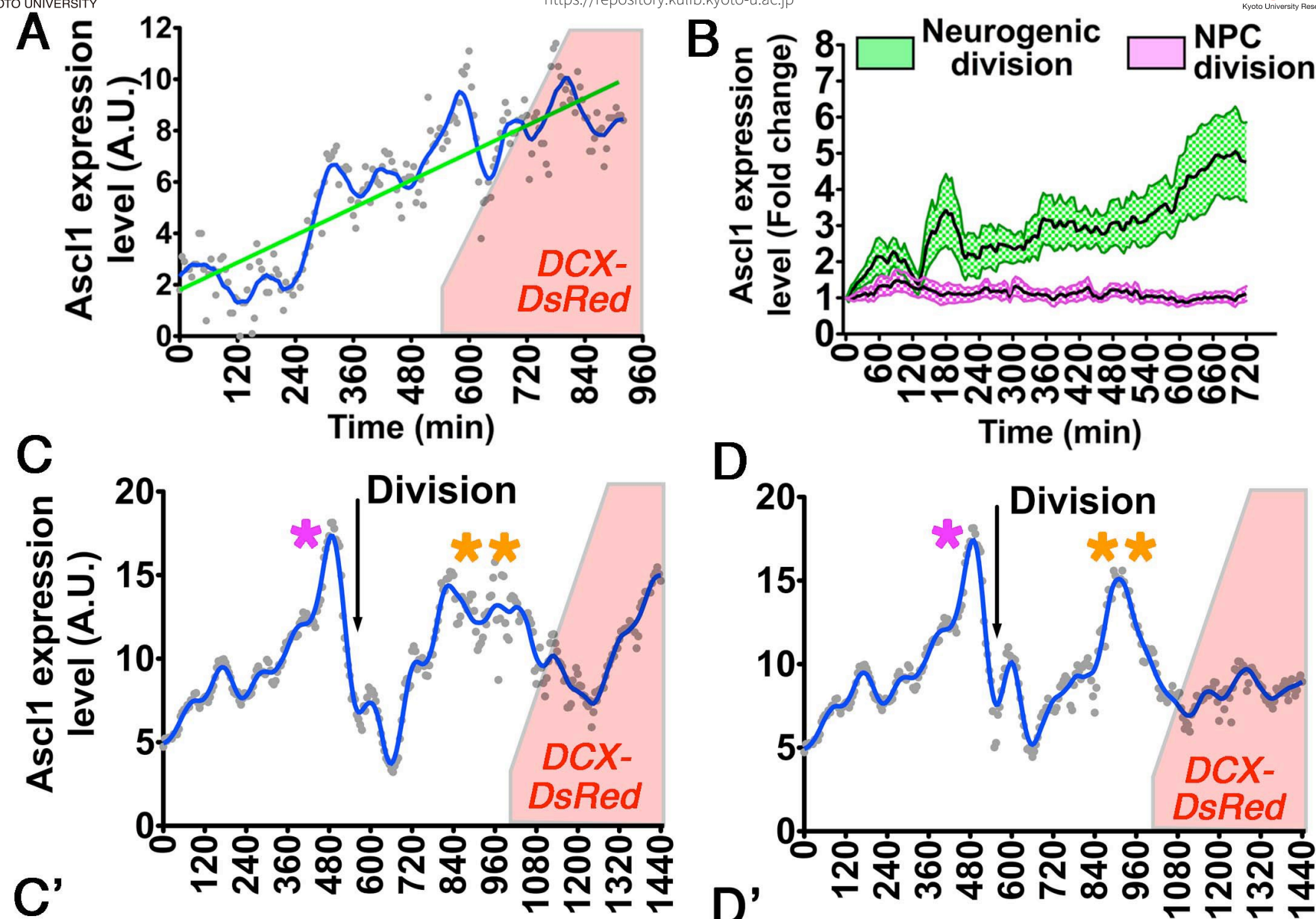

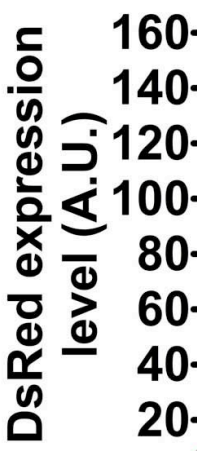

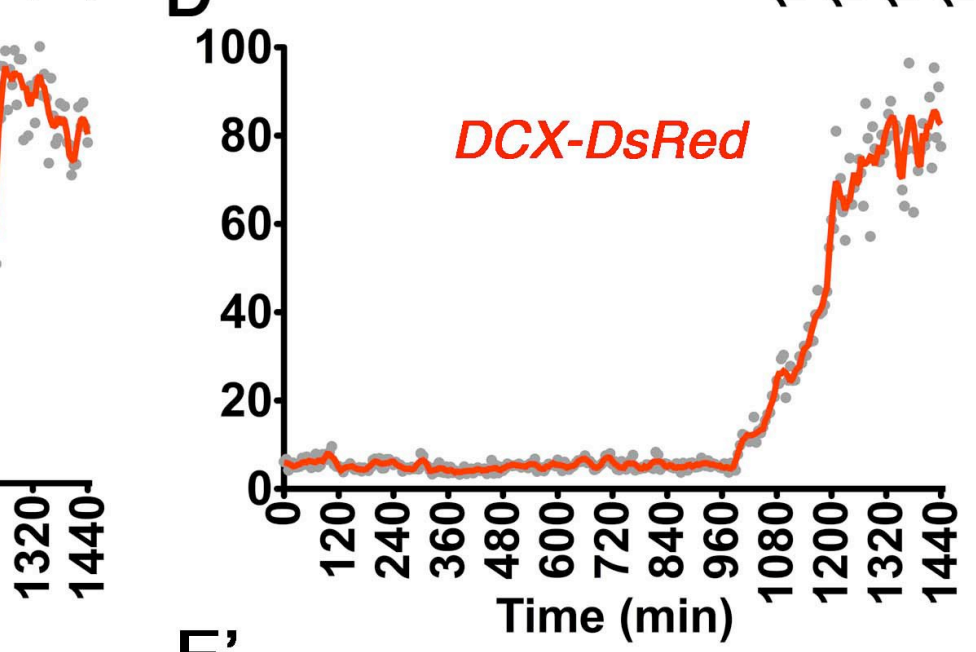

E

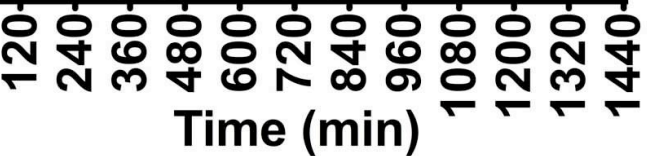
$E^{\prime}$
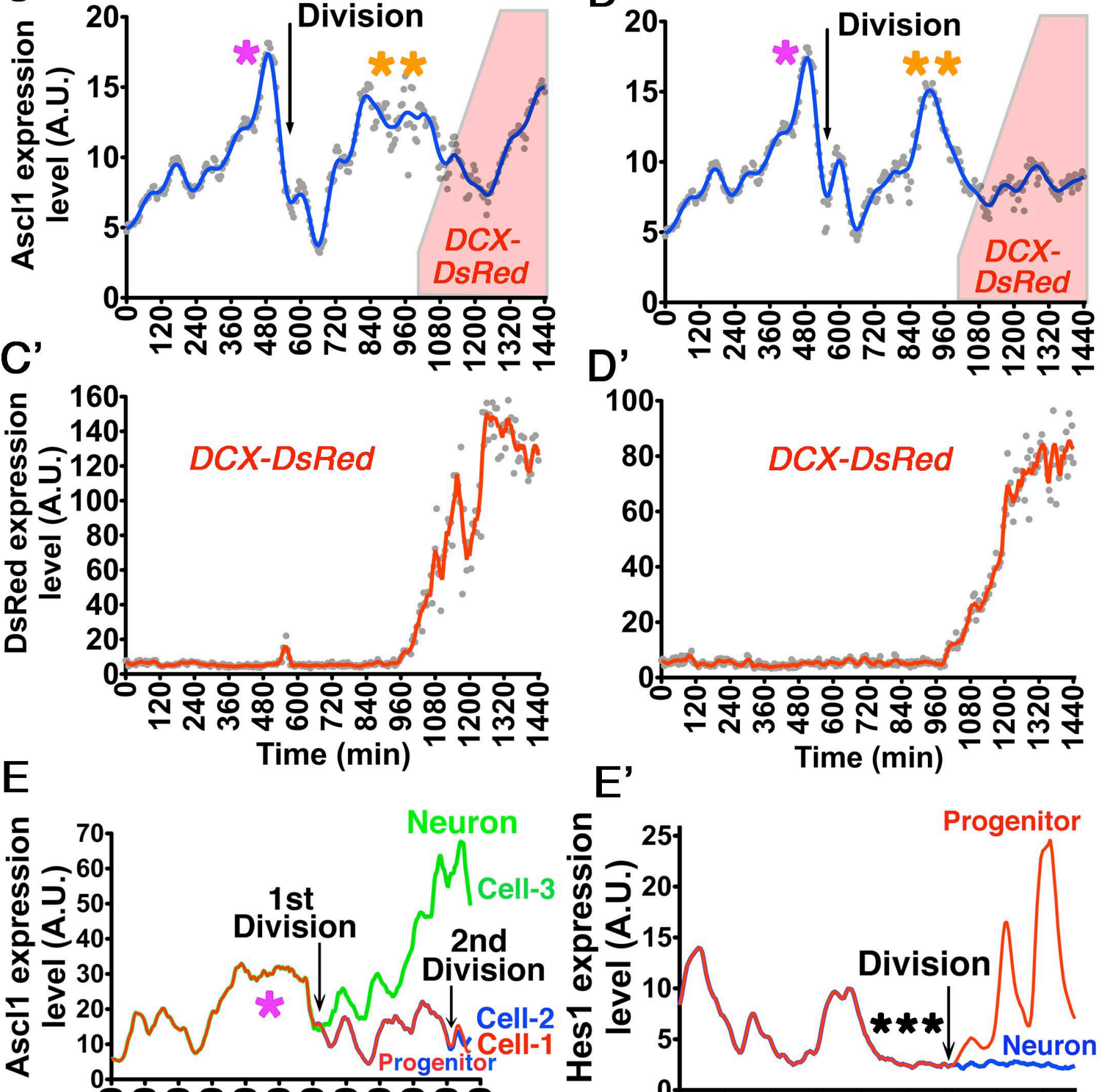

운잉ㅇㅇㅇㅇㅇㅇㅇㅇㅇㅇㅇㅇㅇㅇㅇㅇㅇ N Time (min)

음

Progenitor

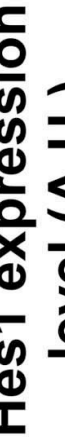
25
20.
5
10
5.
0

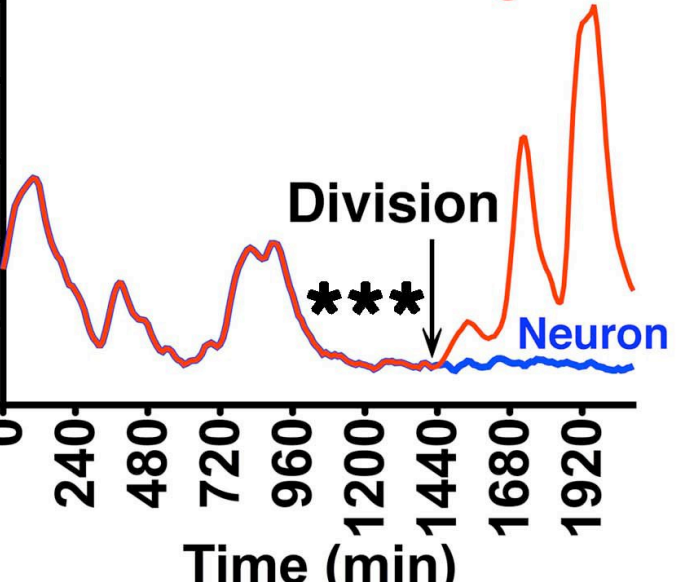



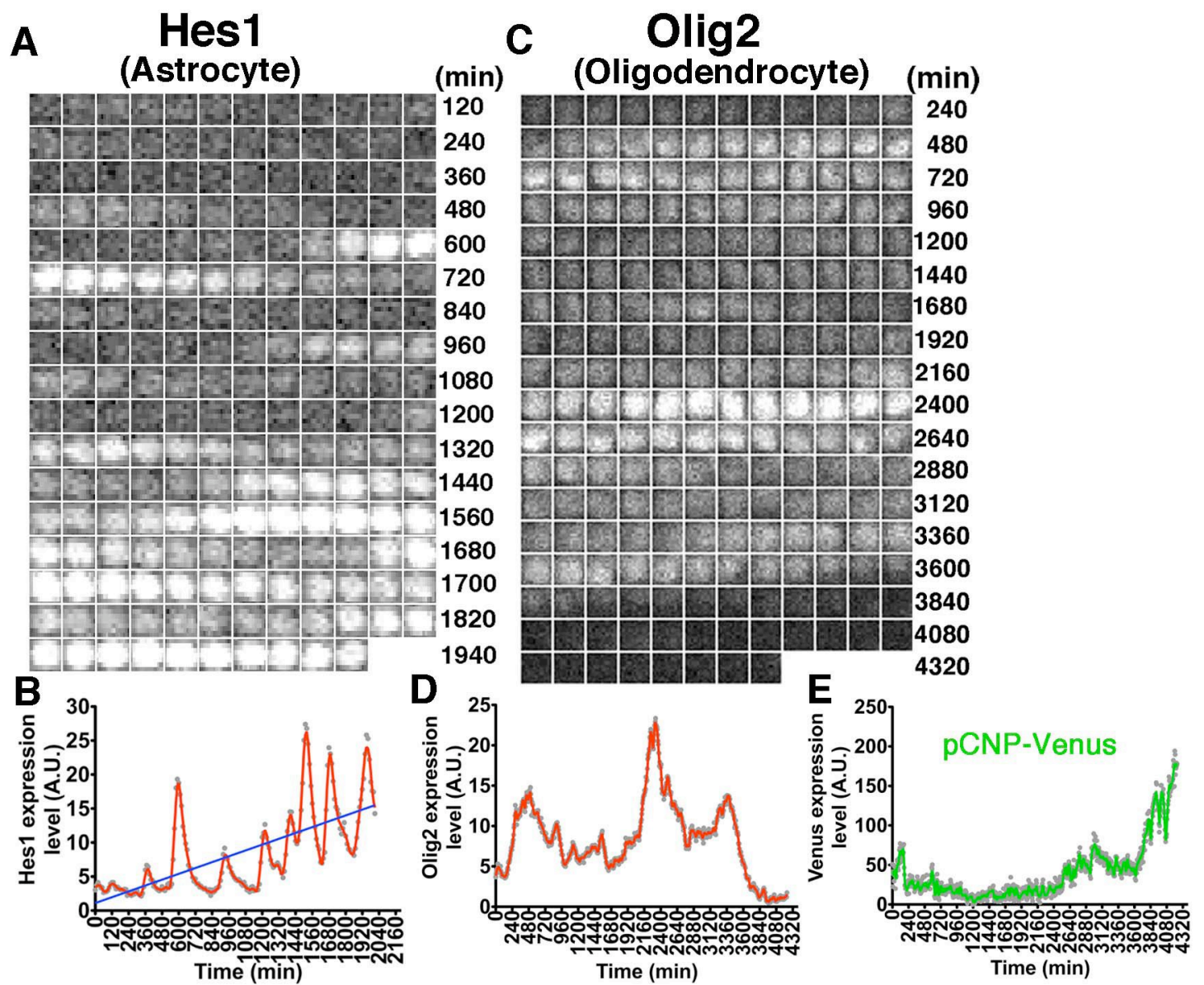

Figure 4 


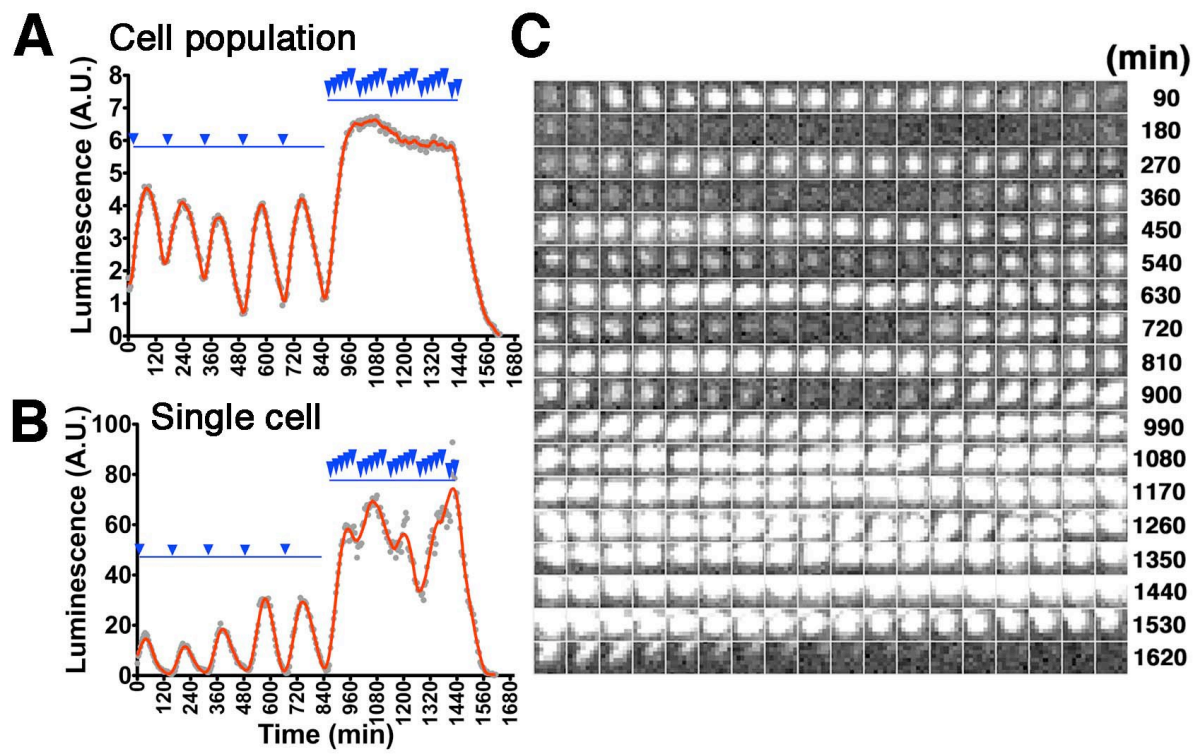

\section{$\mathbf{L}$}

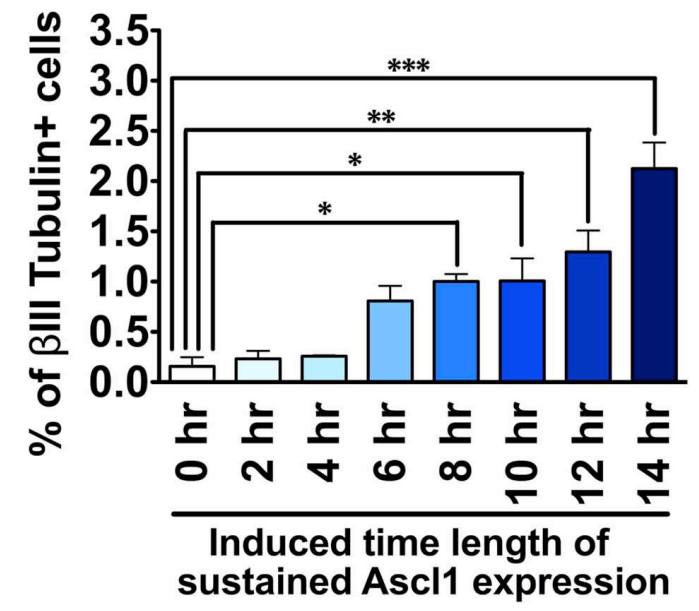

Light-induced oscillatory expression (Ascl1-KO NPC)
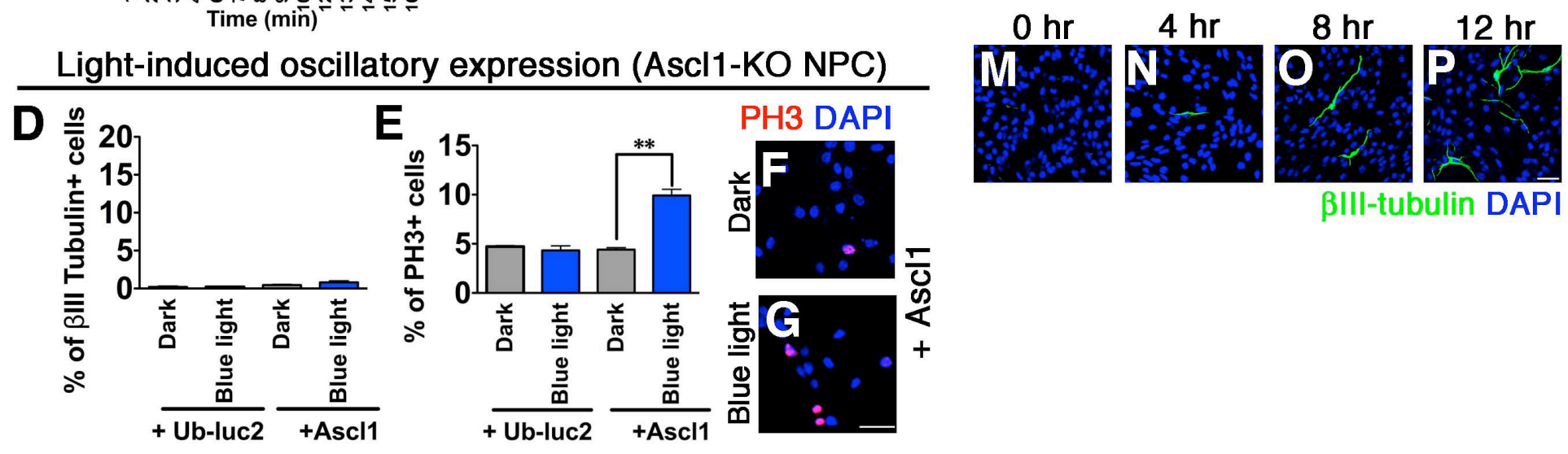

$\beta$ III-tubulin DAPI

Light-induced sustained expression (Ascl1-KO NPC)

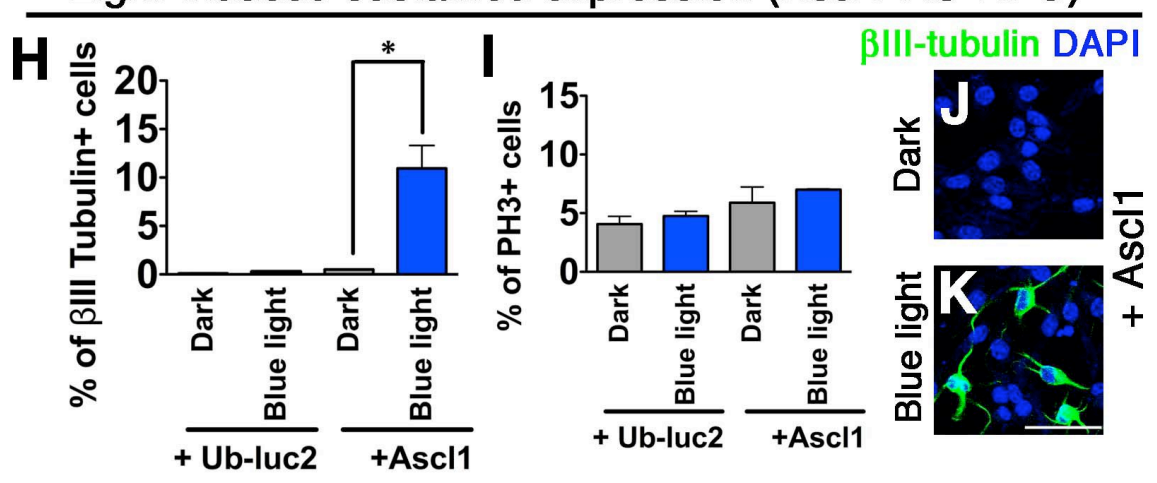

Figure 5 


\section{Supplementary Materials for}

\section{Oscillatory control of factors determining multipotency and fate in mouse neural progenitors}

Itaru Imayoshi, Akihiro Isomura, Yukiko Harima, Kyogo Kawaguchi, Hiroshi Kori, Hitoshi Miyachi, Takahiro K. Fujiwara, Fumiyoshi Ishidate, and Ryoichiro Kageyama*

correspondence to:

rkageyam@virus.kyoto-u.ac.jp \& iimayosh@virus.kyoto-u.ac.jp

\section{This PDF file includes:}

Materials and Methods

Figs. S1 to S31

Tables S1

Captions for Movies S1 to S6

References

Other Suplementary Materials for this manuscript includes the following:

Movies S1 to S6 


\section{Materials and Methods}

\section{Plasmid construction}

To analyze the promoter activity, the ubiquitinated luciferase (Ub-luc) was used as a reporter, as previously described $(38,39)$. This luciferase was fused at its $\mathrm{N}$-terminus to one copy of a mutant ubiquitin $(\mathrm{G} 76 \mathrm{~V})$ that resists cleavage by ubiquitin hydrolases. The resultant Ub-Luc is extremely unstable with a half-life of less than $10 \min (38,39)$. The optimized firefly luciferase luc2 (Promega) was used for most Ub-luc constructs. For two-color simultaneous imaging of two promoter activities in single cells, Eluc from Pyrearinus termitilluminans (TOYOBO) and Red luc from Phrixothrix hirtus (TOYOBO) were used. For easier single-cell tracking, four copies of nuclear localization signal (NLS) of SV40 large T-antigen were attached to Ub-luc. This NLS-Ub-Luc coding sequence was inserted into the reporter constructs that contain the 5'-flanking sequences and the downstream sequences including the 3'-UTR regions.

For the Hes5 reporter construct, 3-kb of the 5'-flanking sequence and 2-kb of downstream sequence were used. For the Rosa26 reporter construct, 0.8-kb of the 5'flanking sequence of the Rosa26 locus and SV40 late poly-A adenylation sequence were used. For the Nestin reporter construct, the 5.8-kb promoter and second intron enhancer sequence of Nestin, and SV40 late poly-A adenylation sequence were used. For the RBPjk-activity reporter construct, four tandem copies of the RBPjk-binding consensus sequence were placed upstream of the SV40 basal promoter, and $0.5-\mathrm{kb}$ of downstream sequence of the Hes1 gene was placed downstream of Ub-luc.

In the pCNP-Venus construct, gap-Venus-T2A-Venus coding sequence was subcloned into the CNP expression cassette, which has a 3.9-kb fragment of the promoter region of CNP (2',3'-Cyclic-nucleotide 3'-phosphodiesterase) gene, and poly-adenylation sequence of SV40. The detailed cloning strategy and complete sequence of the plasmids are available on request.

\section{Animals}

All animals were handled in accordance with the Kyoto University 'Guide for the Care and Use of Laboratory Animals'. Time-pregnant females were maintained in a $12 \mathrm{hr}$ light/dark cycle and obtained by overnight breeding with males of the same strain. Noon following breeding is considered as E0.5. Rosa26-LoxP-Stop-LoxP-NICD (40), DCXDsRed (41), pCAG-mAG-hGem(1/110) (42), and pCAG-mKO2-hCdt1(30/120) (42) lines were described previously. Transgenic $(\mathrm{Tg})$ or knock-in reporter strains were generated as described below.

\section{Conventional Tg mice}

To prepare the DNA fragments for pronuclear injection, the vector backbone sequences were removed. The constructs were isolated by agarose gel electrophoresis, purified with QIAEX II (Qiagen) and injected into the pronuclei of fertilized one-cell eggs from ICR mice. Typically 200-300 fertilized eggs were injected. Genotypes were determined by PCR of tail DNA. 


\section{BAC Tg mice}

BAC targeting was conducted using BAC recombineering $(43,44)$. BAC clones were selected from the Ensembl database (http://www.ensembl.org), and obtained from the BACPAC Resources Center at Children's Hospital Oakland Research Institute (CHORI). BAC DNA was transferred from DH10B strain to SW105 strain by electroporation. The identity and integrity of these BAC clones were verified by a panel of PCR primers and restriction digestions. For generating N-terminal reporter-fusion BAC Tg constructs, reporter coding sequences without stop codon were amplified by PCR, and cloned into pBluescript II SK+ plasmid. HA and flag epitope tags were attached to the $\mathrm{N}$-terminus of Luc2 and Venus, respectively. In front of the reporter coding sequences, frt-PGK-EM7-Neo-frt cassette was inserted. The Neo gene is driven by both the PGK promoter for G418 selection in ES cells and the EM7 promoter for Kan selection in Escherichia coli. A BAC targeting vector was generated for each gene by cloning 300-500-bp homology arms from the gene into a reporter plasmid, flanking the frt-Neo-frt-reporter cassette. For recombineering, BAC targeting cassettes were excised by restriction digestion, and electroporated into competent SW105 cells containing the BAC clone of interest. Targeted BAC clones were selected for KanR, and correctly targeted BAC clones were identified by a panel of PCR primers and restriction digestions. frt-Neo-frt selection cassette was removed by arabinose-mediated transient FLP recombinase expression in SW105 $(43,44)$. BAC DNA was purified with a NucleoBond Xtra Midi kit (Macherey-Nagel) and injected into the pronuclei of fertilized one-cell eggs from ICR mice. Typically 300-400 fertilized eggs were injected. Potential founder animals were screened by PCR of tail DNA. Founder lines having a single copy of BAC transgene were selected by real-time PCR and used for subsequent analysis.

\section{Knock-in mice}

Generation of N-terminal reporter-fusion knock-in constructs was conducted using BAC recombineering. We used the pMCS-DTA retrieval vector (Gift from Dr. Kosuke Yusa, Osaka University, Japan) as the backbone of our knock-in vectors. pMCS-DTA contains the diphtheria toxin fragment-A (DTA) gene driven by the MC1 promoter for negative selection in ES cells. Knock-in cassette fragments were retrieved from modified BAC clones into pMCS-DTA by recombineering. BAC targeting was performed similarly to that of transgene construction of BAC Tg mice as described above. The first exon and the initial part of the first intron sequences were inserted between reporter (Venus or ELuc) coding sequence and the frt-PGK-EM7-Neo-frt cassette, so that frtPGK-EM7-Neo-frt was inserted into the first intron region. A BAC targeting vector was generated for each gene by cloning 300-500-bp homology arms from the gene into a reporter plasmid. Targeted BAC clones were selected for KanR, and confirmed by a panel of PCR primers and restriction digestions. A correctly targeted BAC clone was used for generating the knock-in construct by the BAC retrieval method (43). The 5'- and 3'-homology arms in the retrieval vector were designed such that between 3- and 8-kb DNA segments flanking the reporter-frt-PGK-EM7-Neo-frt cassette in the BAC clone 
were subcloned into pMCS-DTA. The total length of homology (3- and 8-kb on either side) was sufficient for gene targeting in ES cells (TT2). The shorter homology arm was used to design PCR-based screening for targeted ES cells. Southern blot hybridization was performed to confirm successful homologous recombination. Chimeric mice were produced from successfully targeted ES cell clones by aggregation with ICR embryos. Germ line transmission of the targeted allele was assessed by PCR of tail DNA.

Monolayer neural progenitor cell (NPC) culture

NPC culture was prepared and maintained using NS cell culture method, as described previously (30). Basal forebrain regions, including the medial, lateral and caudal ganglionic eminences, were excised from perinatal mice, and digested with Papain (Worthington) for $20 \mathrm{~min}$ at $37^{\circ} \mathrm{C}$ and dissociated completely by pipetting. Papain inhibitor was added, and then the cells were spun down, and resuspended in serum-free culture medium (NS-A media (Euroclone) plus N2 supplement (R\&D Systems), $10 \mathrm{ng} / \mathrm{ml}$ of both basic FGF and EGF (Invitrogen), and penicillin/streptomycin). Culture dishes were coated with poly-L-ornithine (Sigma) and fibronectin (R\&D Systems). In this study, acutely dissociated NPCs or established NS cells (at least 10 passages and 1 cryopreservation) were used. Detailed protocols for routine handling of NS cells were previously described (30).

Neuronal, astrocyte and oligodendrocyte differentiation of NPCs

Differentiation of NS cells was induced as described previously (30). For neuronal differentiation, NS cells were harvested using Papain to detach cells and 0.5-1.0 x 10 cells were re-plated into a poly-L-ornithine/fibronectin coated $35 \mathrm{~mm}$ dish in NS-A medium supplemented with $0.5 \mu \mathrm{M}$ retinoic acid (Sigma), N2, and B27 supplement. Rapid differentiation of NS cells to GFAP-positive astrocytes occurred within 2 days of exposure of NS cells to $80 \mathrm{ng} / \mathrm{ml}$ LIF (Millipore) and 80ng/ml BMP4 (R\&D Systems). For oligodendrocyte differentiation, NS cells were harvested using Papain to detach cells and $0.5-1.0 \times 10^{4}$ cells were re-plated into a poly-L-ornithine/fibronectin coated $35-\mathrm{mm}$ dish in NS-A medium supplemented with N2, 10ng/ml PDGF (R\&D Systems) and 30ng/ml 3,3,5-triiodothyronine (T3; Sigma).

\section{NPC transfection}

NS cells were nucleofected using an AAD-1001 nucleofector device with mouse NS cell nucleofector solution (Amaxa), program A-033, and $5 \mu \mathrm{g}$ of DNA per $1.0 \times 10^{6}$ cells. Cells were analyzed $48 \mathrm{hr}$ after nucleofection.

\section{Lentiviral vector production and transduction of NPCs}

Coding sequences of gene of interest were inserted into multiple cloning sites of CSII-EF-MCS (45). Lentiviral particles were produced via calcium phosphate cotransfection of $293 \mathrm{~T}$ cells with the packaging plasmids in the same procedure as previously described (45). Supernatants were collected starting from $24 \mathrm{hr}$ after the transfection for $36 \mathrm{hr}$ and concentrated by centrifugation at $6,000 \mathrm{~g}$ for $16 \mathrm{hr}$. The 
resulting viral pellet was resuspended in PBS at 1/500th of the original volume, and the viral aliquot was then frozen. Viral titers were approximately $10^{9}$ infectious units $/ \mathrm{ml}$. Cultured NS cells were infected by purified lentiviral particles with MOI = approximately 10-20, and analyzed $48 \mathrm{hr}$ after infection.

Immunocytochemistry

Cells were washed by phosphate-buffered saline (PBS) and fixed with $4 \%$ paraformaldehyde (PFA)/PBS for $20 \mathrm{~min}$ at room temperature. Fixed cells were washed by PBS, then blocked and permeated with 5\% normal donkey serum (NDS) and $0.1 \%$ Triton X-100/PBS at room temperature for $20 \mathrm{~min}$, incubated with primary antibodies diluted in PBS containing $1 \%$ NDS overnight at $4{ }^{\circ} \mathrm{C}$, washed with PBS, and then incubated with regular secondary antibodies conjugated to Alexa 488, Alexa 594 or Alexa 647 (1:200, Invitrogen) for $1 \mathrm{hr}$ at room temperature. Stained cells were photographed with LSM510 or LSM780 confocal microscopes (Zeiss).

Immunohistochemistry

Embryos were fixed in $4 \% \mathrm{PFA} / \mathrm{PBS}$ for $3 \mathrm{hr}$ or overnight at $4{ }^{\circ} \mathrm{C}$, washed in icecold PBS, equilibrated in $20 \%$ sucrose/PBS at $4{ }^{\circ} \mathrm{C}$, embedded in OCT compound (Sakura Finetek), and frozen at $-80^{\circ} \mathrm{C}$. Pups and adult mice were deeply anesthetized and perfused transcardially with $30 \mathrm{ml}$ of PBS and $30 \mathrm{ml}$ of $4 \% \mathrm{PFA} / \mathrm{PBS}$. Brains were postfixed in the perfusing solution overnight at $4^{\circ} \mathrm{C}$ and then cryoprotected for $48 \mathrm{hr}$ in $20 \%$ sucrose in PBS. Brains were embedded in OCT compound, and frozen at $-80^{\circ} \mathrm{C}$. Sections were made at $16-\mu \mathrm{m}$ thickness using a cryostat, and incubated in 5\% NDS and $0.1 \%$ Triton $\mathrm{X}-100 / \mathrm{PBS}$ at room temperature for $1 \mathrm{hr}$, and with primary antibodies diluted in $0.1 \%$ Triton $\mathrm{X}-100 / \mathrm{PBS}$ containing $1 \%$ NDS overnight at $4^{\circ} \mathrm{C}$. Sections were washed with PBS, and incubated with secondary antibodies conjugated to Alexa 488, Alexa 594 or Alexa 647 (1:200, Invitrogen) for $2 \mathrm{hr}$ at room temperature. Then, sections were mounted with Fluormount-G (Southern Biotech) and photographed with LSM510 or LSM780 confocal microscope (Zeiss). Immunostaining for NICD was done, as previously described (46).

\section{Antibodies}

The following primary antibodies (final dilution for immunohistochemistry and source) were used: rabbit anti-GFP (1:500; Invitrogen A11122), rat anti-GFP (1:200; Nacalai tesque GF090R), rabbit anti-DsRed (1:500; Clontech 632496), rat anti-HA (1:200; Roche 3F10 clone), rabbit anti- $\beta$ III-Tubulin (1:500; Covance A11122), mouse

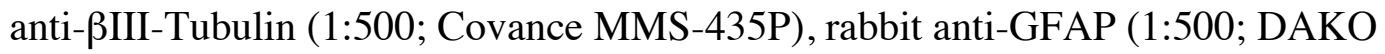
Z033429), mouse anti-GFAP (1:500; Sigma G3893), rabbit anti-CNPase (1:500; CST 5664S), mouse anti-Nestin (1:500; BD Pharmingen 556309), rabbit anti-PH3 (1:500; Millipore 06-570), rabbit anti-Hes1 (1:500), guinea pig anti-Hes1 (1:500), mouse antiAsc11 (1:500; BD Pharmingen 556604), rabbit anti-Olig2 (1:500; IBL 18953), mouse anti-Olig2 (1:500; Millipore MABN50), rabbit anti-Sox2 (1:500; Millipore AB5603), and rabbit anti-NICD (1:500; CST 4147S). 
RNA in situ hybridization

RNA in situ hybridization was performed using digoxigenin-labeled Luc2 and Eluc antisense RNA probes, as described previously (47).

Fluorescence-activated cell sorting (FACS)

NPCs were dissociated with Papain (Worthington), washed with DMEM/F12 supplemented with N2 supplement (R\&D Systems) and $0.1 \%$ BSA, and collected by centrifugation. Large clumps of cells were removed using a cell strainer (BD Biosciences). Cells were sorted and analyzed on a flow cytometer (ARIA II; BD Biosciences). The fluorescence activities of Venus and mCherry were detected in the FITC $(\mathrm{Ex}=488 \mathrm{~nm})$ and Grn PE $(\mathrm{Ex}=561 \mathrm{~nm})$ channel, respectively. NPCs were sorted to DMEM/F12 supplemented with N2 supplement (R\&D Systems) and 0.1\% BSA, and collected by centrifugation. For differentiation assays, NPCs were directly sorted to serum-containing differentiation medium; DMEM/F12 supplemented with N2 supplement (R\&D Systems), B27 supplement (Invitrogen), 10\% fetal bovine serum and penicillin/streptomycin.

\section{Neurosphere assay}

Neurosphere assays were performed from FACS-purified NPCs as described previously (48). Cells sorted by FACS were collected by centrifugation and re-suspended in neurosphere medium (N2 supplement (R\&D Systems), B27 supplement (Invitrogen), $20 \mathrm{ng} / \mathrm{ml}$ of EGF (Invitrogen) and 20ng/ml of bFGF (Invitrogen), and penicillin/streptomycin). Cells were counted and plated in uncoated 35-mm dishes (50,000 cells per dish). Primary neurospheres were counted 7 days after plating, and neurospheres were passaged by harvesting them by centrifugation (200g for $5 \mathrm{~min}$ ) and triturating them in $100 \mu \mathrm{l}$ of medium with an automatic pipetter (P200 Gilson).

\section{Differentiation assay}

Cells sorted by FACS were collected by centrifugation and re-suspended in serumcontaining differentiation medium, and then seeded in poly-L-ornithine- and fibronectincoated 35-mm dishes (50,000 cells per dish). After 7 days in culture, cells were fixed and analyzed by immunocytochemistry. Data are presented as mean \pm SEM from at least $n=6$ independent experiments counting $>200$ cells from randomly selected fields per each experiment.

\section{Luminescence imaging of NPCs}

NPCs carrying reporters were plated to coated 35-mm glass-base dishes at 50-60\% confluence and incubated at $37^{\circ} \mathrm{C}$ and $5 \% \mathrm{CO}_{2}$. Cells were imaged just after plating or next day. $1 \mathrm{mM}$ luciferin was added to the culture medium. Bioluminescence images were acquired by an upright microscope (IX81; Olympus) with x60 dipping objective (1.42 NA). Digital images were acquired using a cooled CCD camera (iKon-M DU934P-BV, Andor). The filters and camera control were adjusted automatically using software (Meta 
Morph; Universal Imaging Corp.). Stray light was cut off by turning off the electric system. The imaging system was used in a dark room. For dual-color bioluminescence imaging, we used only bandpass emission filters (FF01-510/84-25 for Eluc and BLP01635R-25 for Red luc, Semrock). Luciferase-reporter mice were crossed with DCX-DsRed (41) Tg mice for monitoring neuronal differentiation or with pCNP-Venus mice for monitoring oligodendrocyte differentiation, and NPCs were collected from the double Tg mice. To monitor the cell cycle phases, luciferase-reporter mice were crossed with pCAG-mAG-hGem(1/110) (42) \& pCAG-mKO2-hCdt1(30/120) (42) mice, and NPCs were collected from the triple $\mathrm{Tg}$ mice.

\section{Organotypic slice culture for luminescence imaging}

Time-lapse imaging of brain slices was performed, as described previously (49). Embryos were harvested in PBS, and brains were isolated in DMEM/F-12 supplied with $10 \mathrm{mM}$ HEPES. Brain tissue was immediately transferred into a silicon rubber-coated dish with DMEM/F-12 containing 10mM HEPES, which was previously bubbled with $100 \% \mathrm{O}_{2}$ for $10-15$ min on ice. Meninges were removed, and coronal brain slices (100$200-\mu \mathrm{m}$ thickness) were manually prepared by ophthalmic microsurgical knife (Alcon). Slices of basal telencephalon were transferred to a glass-base dish containing slice culture medium (DMEM/F-12 supplemented with N2, B27, 20ng/ml EGF, 20ng/ml bFGF, 5\% horse serum, and $5 \%$ fetal bovine serum). Slices were immersed in about $200 \mu 1$ of type Ia collagen (Cellmatrix, Nitta Gelatin) (diluted to $1.5 \mathrm{mg} / \mathrm{ml}$ with DMEM/F-12 and neutralizing buffer according to the manufacturer's instructions and kept on ice until use) at room temperature. After 20 -min incubation at $37^{\circ} \mathrm{C}$, slices were cultured at $37^{\circ} \mathrm{C}$ in $1 \mathrm{ml}$ of slice culture medium containing $1 \mathrm{mM}$ luciferin. Then, the dish was placed on a stage of inverted microscope and maintained at $37^{\circ} \mathrm{C}$ in $5 \% \mathrm{CO}_{2}$ and $45 \% \mathrm{O}_{2}$. Bioluminescence from the sample was acquired using the CCD camera, as described above.

\section{Fluorescence imaging of NPCs}

Fluorescent live cell images were acquired using a confocal laser scanning microscope, LSM 780 (Carl Zeiss), equipped with the spectral GaAsP array detector. NPCs were plated to coated $35-\mathrm{mm}$ glass-base dishes at 50-60\% confluence and incubated at $37^{\circ} \mathrm{C}$ and $5 \% \mathrm{CO}_{2}$. Venus and mCherry fluorescent protein were activated by 514-nm Argon laser and 561-nm DPSS laser, respectively. Images were acquired with the photon-counting mode. Spectral imaging was performed using a 32-channel GaAsP detector of the LSM 780 system, and specific Venus or mCherry fluorescent signal was separated from autofluorescence by applying linear unmixing algorithms (Carl Zeiss).

Image analysis and quantification

Image analysis was performed using ImageJ software and custom plug-ins. Custom written code of ImageJ plug-ins used in this study is available on request.

For analyzing an image sequence file of bioluminescence imaging, 'Spike-noise filter' was applied to a stack file to remove noise signals caused by cosmic rays. CCD 
readout noise was also removed by 'Temporal background reduction filter'. In this normalization procedure, the background value measured in the outside of the imaging regions for each time-flame was subtracted from the signal intensity. Tracking individual cells and quantification of bioluminescence signals were conducted with 'Circadian Gene Expression (CGE)' (http://bigwww.epfl.ch/sage/soft/circadian/). In some experiments, nuclear localized mCherry was expressed by nucleofection or lentivirus vectors, and used to detect and track moving cells. Average signal intensity inside the nucleus were measured, illustrated and analyzed by Prism 5.0 software (GraphPad Software).

Fixed monolayer NPCs were immunostained, and images were acquired with a Zeiss Axio Observer.Z1 inverted microscope with a 20x Plan-Apochromat objective (0.8 NA) or 40x Plan-Apochromat objective (1.3 NA). Automated segmentation was performed, and data was analyzed using custom written code (Static particle analyzer) in ImageJ. Histogram and scatter plot of protein levels in single cells were illustrated and analyzed by Prism 5.0 software.

Ascl1 expression by light-switchable gene expression system

Codon-optimized GAVPO (36) (blue light-activatable Gal4 transcriptional activator, hGAVPO) was subcloned into pEF-BOS expression vector for ex utero electroporation and CSII-EF-MCS-IRES2-Bsd plasmid (45) for lentivirus production. Bsd is the resistance for blasticidin. CSII-EF-MCS was digested with AgeI to remove EF promoter, and 5x UAS sequence and 3'-untranslated region (UTR) of mouse Ascll gene was cloned in the opposite orientation to the LTR-mediated transcription. NLS-Ub-luc2 or Ascl1 coding sequences were inserted immediately after the $5 x$ UAS sequence.

Using the CSII-EF-hGAVPO-IRES2-Bsd, CSII-UAS-NLS-Ub-luc2 or CSII-UASAscl1 plasmids, lentiviral particles were produced and purified as described above. Established wild-type or Ascl1-mutant NS cells were co-infected with EF-hGAVPOIRES2-Bsd\&UAS-NLS-Ub-luc2 or EF-hGAVPO-IRES2-Bsd\&UAS-Ascl1 lentiviral

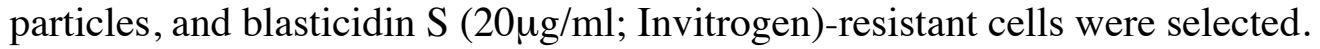
Blasticidin-selected cells were passaged 3- to 5-times before analysis.

For generating oscillatory expression of transgene, blue-light was illuminated for 1 min with a 3-hr interval. For achieving sustained expression of transgene, blue-light was illuminated for 2 min with a 30-min interval, unless otherwise noted. Blue light was generated by pE-2 LED excitation system (CoolLED) equipped with 470-nm LAM and illuminated to cells through the $\mathrm{x} 40$ objective lens (UPLFLN40XO). Light-intensity was adjusted to 1 to $7 \%$. Measured light power $(\mathrm{LED}=1 \%)$ was approximately $200 \mu \mathrm{mol} / \mathrm{m}^{2} / \mathrm{s}$ in this experimental condition. Blue-light illumination was automatically controlled using Meta Morph software. In the analysis of cell-proliferation or differentiation analysis, blue-light was generated by LEDB-SBOXH (OptoCode) in $\mathrm{CO}_{2}$-incubators. Light power was changed from $0.16 \mu \mathrm{mol} / \mathrm{m}^{2} / \mathrm{s}$ to $1.11 \mu \mathrm{mol} / \mathrm{m}^{2} / \mathrm{s}$. To analyze the effects of oscillatory or sustained expression on cell proliferation and neuronal differentiation, plated NPCs were illuminated for $72 \mathrm{hr}$ and analyzed by immunocytochemistry. Data are presented as mean \pm SEM from at least $n=3$ independent experiments counting $>200$ cells from randomly selected fields per each experiment. 
Ascl1-light inducible system was introduced into dorsal telencephalon progenitors. pEF-mCherry-NLS, pEF-hGAVPO and CSII-UAS-NLS-Ub-luc2/CSII-UAS-Asc11 plasmids were mixed at 2:3:5 ratio, and co-transfected into E14.5 dorsal telencephalon progenitors by ex utero electroporation. Plasmid DNA $(2.5 \mu \mathrm{g} / \mu \mathrm{l})$ was microinjected into a telencephalic ventricle, and ex utero electroporation $(5$ pulses, $50 \mathrm{mV}$, square wave generator (CUY21, BEX), 5-mm paddle electrodes) was performed for transfection of plasmids into progenitors at the ventricular surface of the neocortex. Brains were immediately dissected, embedded in 3\% low-melting point agarose, cut into $250-\mu \mathrm{m}$ organotypic slices with a vibratome (VT1000; Leica), transferred to 35-mm well culture insert (353090, BD Falcon), and cultured in slice culture medium. Slices were incubated at $37^{\circ} \mathrm{C}, 5 \% \mathrm{CO}_{2}$ under the periodic blue-light illumination. For analyzing cell fate specification of transfected NPCs, electroporated hemispheres were immediately dissociated by Papain and plated to coated 35-mm glass-base dishes at 50-60\% confluence, and incubated at $37^{\circ} \mathrm{C}$ and $5 \% \mathrm{CO}_{2}$.

\section{Statistical Analysis}

Statistical analyses were performed with Prism 5.0 software (GraphPad). $P$ values less than 0.05 were considered to be significant. Statistical methods used in the analysis were described in figure legends. 


\section{Supplementary Text:}

Hes1 represses the expression of itself and Dll1 (a ligand for Notch signaling) of the same cell, while Hes1 expression is up-regulated by Dll1 of the neighboring cells (Fig. 1). We show through a simple mathematical model that this regulatory pathway generates oscillation and suppression of Hes1 expression in the interacting cells. The model for the dynamics of Hes1 and Dll1 is given by the following equations,

$$
\begin{aligned}
\dot{H}_{i}(t) & =V_{\mathrm{H}} \frac{D_{\langle i\rangle}(t)^{2}}{D_{\langle i\rangle}(t)^{2}+K_{\mathrm{HD}}^{2}} \frac{K_{\mathrm{H}}^{2}}{H_{i}\left(t-\tau_{\mathrm{H}}\right)^{2}+K_{\mathrm{H}}^{2}}-d_{\mathrm{H}} H_{i}(t), \\
\dot{D}_{i}(t) & =V_{\mathrm{D}} \frac{K_{\mathrm{DH}}^{2}}{H_{i}(t)^{2}+K_{\mathrm{DH}}^{2}}-d_{\mathrm{D}} D_{i}(t), \\
D_{\langle i\rangle}(t) & = \begin{cases}D_{j}(t) & \text { (two-cell model, } j \neq i) \\
\sum_{j \neq i,\left|\vec{r}_{j}(t)-\vec{r}_{i}(t)\right|<I} D_{j}(t) & \text { (multicell model). }\end{cases}
\end{aligned}
$$

Here, $i, j$ are the labels for cells, which are 1 or 2 in the two-cell model, and $1 \leq i, j \leq N$ in the multicell model. $H_{i}(t)$ and $D_{i}(t)$ are the protein level of Hes1 and Dll1 in cell $i$, respectively. $V_{\mathrm{H}}$ and $V_{\mathrm{D}}$ are the maximum synthesis rates, and $d_{\mathrm{H}}$ and $d_{\mathrm{D}}$ are the degradation rates of the corresponding proteins. $\tau_{\mathrm{H}}$ in Eq. (1) is the time required for Hes1 to affect its own formation in the same cell through the negative feedback loop $(50) . D_{\langle i\rangle}(t)$ is the total Dll1 input that cell $i$ receives at time $t$ from neighboring cells. In the multicell model, the two-dimensional position of cell $i$ at time $t$ is denoted as $\vec{r}_{i}(t)=\left(x_{i}(t), y_{i}(t)\right)$, and the sum in Eq. (3) is taken over the cells that are in the positions close enough to cell $i$ (length $=\left|\vec{r}_{j}-\vec{r}_{i}\right|<I$ ). $K_{\mathrm{HD}}, K_{\mathrm{H}}$ and $K_{\mathrm{DH}}$ correspond to the typical amount of Hes1 or Dll1 that account for the enhancement or repression. Although it is more realistic to introduce time delays to $D_{\langle i\rangle}(t)$ in Eq. (1) and $H_{i}(t)$ in Eq. (2), we have confirmed that the basic features of the model remain the same in the absence of such time delays when $d_{\mathrm{D}} \lesssim d_{\mathrm{H}}$. We set all

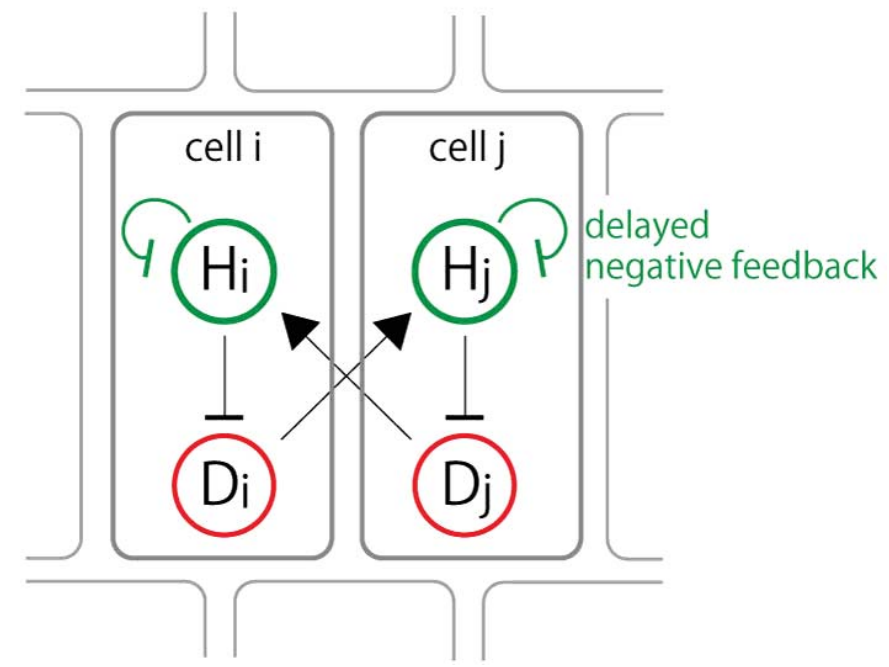

Fig. 1: Schematic structure for the Hes1 and Dll1 regulatory pathway. $H_{i}$ and $D_{i}$ correspond to the protein level of Hes1 and Dll1 in cell $i$, respectively. Cells interact through the Notch signaling activation induced by Dll1. 
the Hill coefficients to be 2, following the notion that Hes1 and Dll1 act as dimers. Assuming larger Hill coefficients does not change the qualitative dynamics.

Cell 1

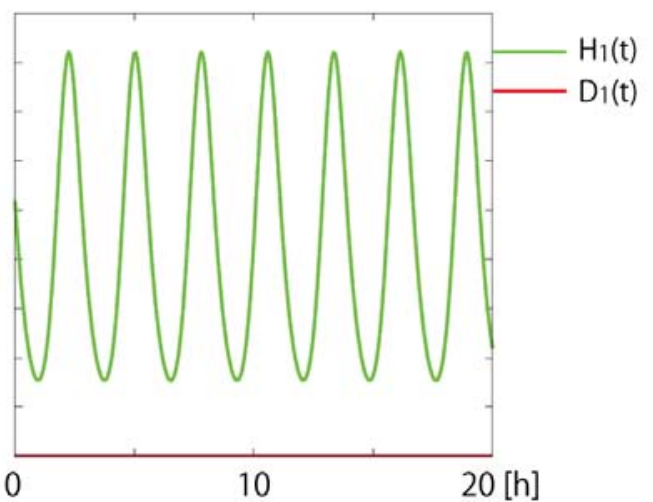

Cell 2

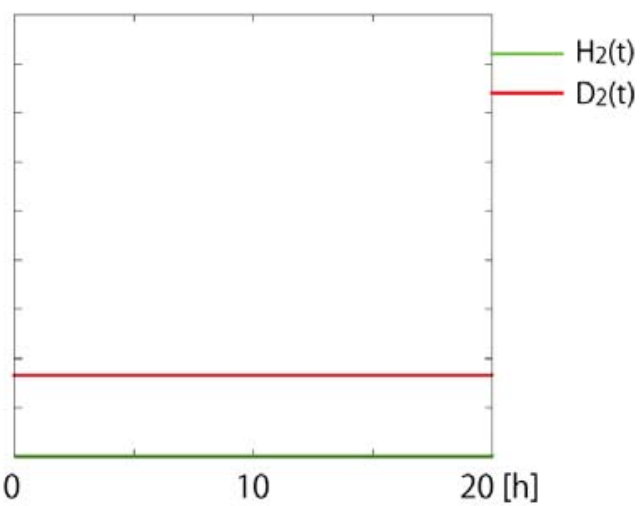

Fig. 2: Numerical simulation of the two-cell model given by Eqs. (1,2). Lines show the time series of protein levels (arbitrary units) after transient time. The Hes1 protein level of one of the cells shows a clear oscillatory behavior with period $\sim 2.7 \mathrm{~h}$, whereas in the other cell it becomes almost quiescent. This behavior is reproduced for any given random initial condition. We used the parameter values $\tau_{\mathrm{H}}=1[\mathrm{~h}], d_{\mathrm{H}}=2.1\left[\mathrm{~h}^{-1}\right]$ (both estimatied from experiments), $d_{\mathrm{D}}=1.5\left[\mathrm{~h}^{-1}\right], V_{\mathrm{H}}=50, V=5$, $K_{\mathrm{HD}}=1, K_{\mathrm{H}}=1.5$, and $K_{\mathrm{DH}}=0.1$.

First, we show the result for the two-cell case (Fig. 2). Although the equations (1) and (2) are symmetric in $i=1,2$, the average level of Hes1 takes two discrete values in the two cells. This is due to the mutual (lateral) inhibition effect of Dll1 through Hes1, and whether cell 1 or 2 keeps the higher Hes1 level is determined by the randomly given initial condition. In Fig. 2, the high Dll1 level in cell 2 activates Notch signaling in cell 1, yielding the high, oscillatory Hes1 expression in cell 1. Such self-sustained oscillation occurs in a delayed feedback model in the condition that $H_{i}(t)$ is sufficiently expressed (51). Dll1 expression in cell 1 is suppressed by the high Hes1 expression, and consequently the Notch signaling level in cell 2 is insufficient to produce abundant Hes1.

Next, we construct the multicell model. Considering that neural progenitor cells move slowly in vivo, we artificially model the motion of each cell by an overdamped Brownian motion in the twodimensional space. We further assume an attracting force between cells, modeled by a linear elastic force with the natural length $L$ and stiffness $k$, to incorporate the fact that cells do not disperse in vivo. The equation of motion is given by,

$$
\begin{aligned}
\dot{\vec{r}}_{i}(t) & =-\frac{\partial}{\partial \vec{r}_{i}} U\left(\vec{r}_{1}, \vec{r}_{2}, \ldots \vec{r}_{N}\right)+\vec{\xi}_{i}(t), \\
U\left(\vec{r}_{1}, \vec{r}_{2}, \ldots \vec{r}_{N}\right) & =\sum_{i, j>i,\left|\vec{r}_{j}(t)-\vec{r}_{i}(t)\right|<C} \frac{1}{2} k\left(\left|\vec{r}_{i}(t)-\vec{r}_{j}(t)\right|-L\right)^{2},
\end{aligned}
$$

where $U\left(\vec{r}_{1}, \vec{r}_{2}, \ldots \vec{r}_{N}\right)$ is the "potential" that mimics the motional interaction between the cells. The potential confines the neighbor cells at a typical distance $L$, which should serve as a model for cell adhesion. The cutoff length $C$ is introduced since cell adhesion occurs only between neighboring cells. $\vec{\xi}_{i}(t)=\left(\xi_{i}^{x}(t), \xi_{i}^{y}(t)\right)$ is the noise, satisfying $\left\langle\xi_{i}^{x}(t) \xi_{j}^{x}(s)\right\rangle=\left\langle\xi_{i}^{y}(t) \xi_{j}^{y}(s)\right\rangle=A \delta_{i j} \delta(t-s)$ and $\left\langle\xi_{i}^{x}(t) \xi_{j}^{y}(s)\right\rangle=0$, with \langle\rangle being the ensemble average. Simulation of this multicell model including cell division (method shown in the caption of Fig. 3) expresses the feature that the random motion 

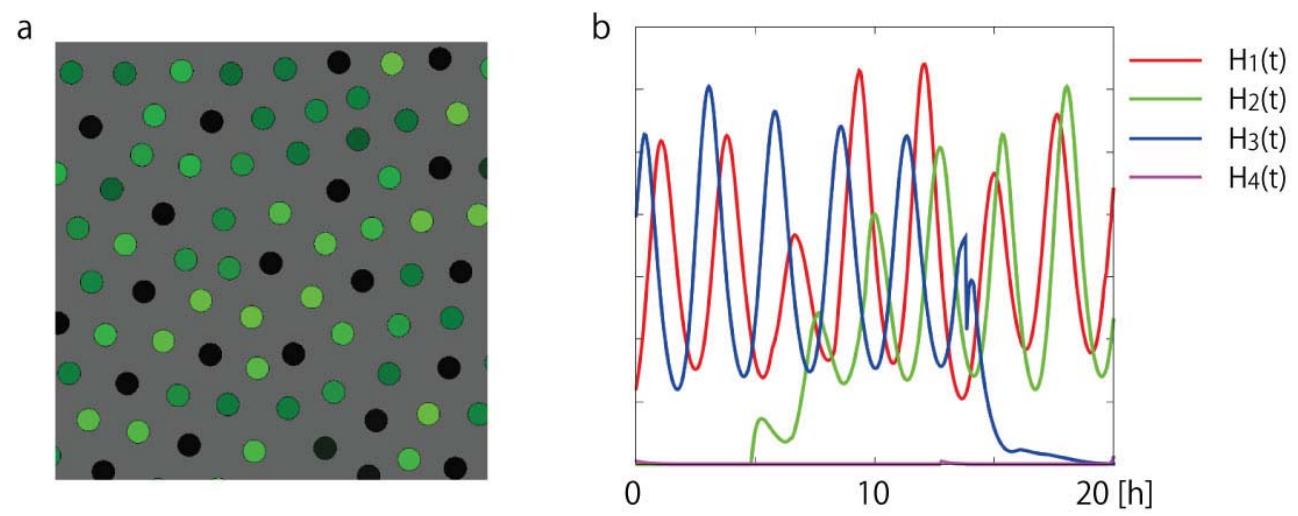

Fig. 3: Numerical simulation of the multicell model [Eqs. (1,2) and Eq. (4)]. We used the same parameter values as those in Fig. 2 for Eqs. $(1,2)$, except for $K_{\mathrm{HD}}$. We set $K_{\mathrm{HD}}=1$, since the cells typically interact with 6 neighbor cells in our multicell model. For the motion of the cells [Eq. (4)], we used the parameters $L=1$ (unit of cell size), $I=C=1.25, A=0.04$, and $k=8$. We initially prepared $N=60$ cells, and assumed that the cells undergo cell division every 24 h (thus $N$ increases with time). In the event of the division, the Hes1 and Dll1 values were equally shared between the two daughter cells. The initial positions of the cells were given randomly in a relatively small region. a) Snapshot of the cell position and Hes1 level (color scale) in the simulation. The typical interval between the center of cells (depicted by the circle with diameter 0.5 ) is approximately $L(=1)$ due to the motional interaction [Eq. (5)]. The green color scale corresponds to the relative level of $H_{i}(t)$ (Hes1); green cells are mostly oscillating, whereas the black cells are in the quiescent phase (low Hes1 and high Dll1 level). b) The typical time courses of Hes1 level in 4 cells in the multicell simulation. Some cells are in the oscillatory phase (cell 1), whereas others stay in the quiescent phase (cell 4). Because the random motion and cell division provide relatively strong perturbation to the cells, some cells experience both the Hes1 oscillatory and quiescent phases during the time course (cells 2 and 3). Cells that are stuck in the quiescent phase may express Ascl1 in a sustained manner, and later differentiate into neurons.

as well as the proliferation of the cells allow single cells to switch back and forth between the Hes1 positive and negative phases (cells 2 and 3 in Fig. 3b), corresponding to the two phases observed in the previous two-cell model (Fig. 2). 

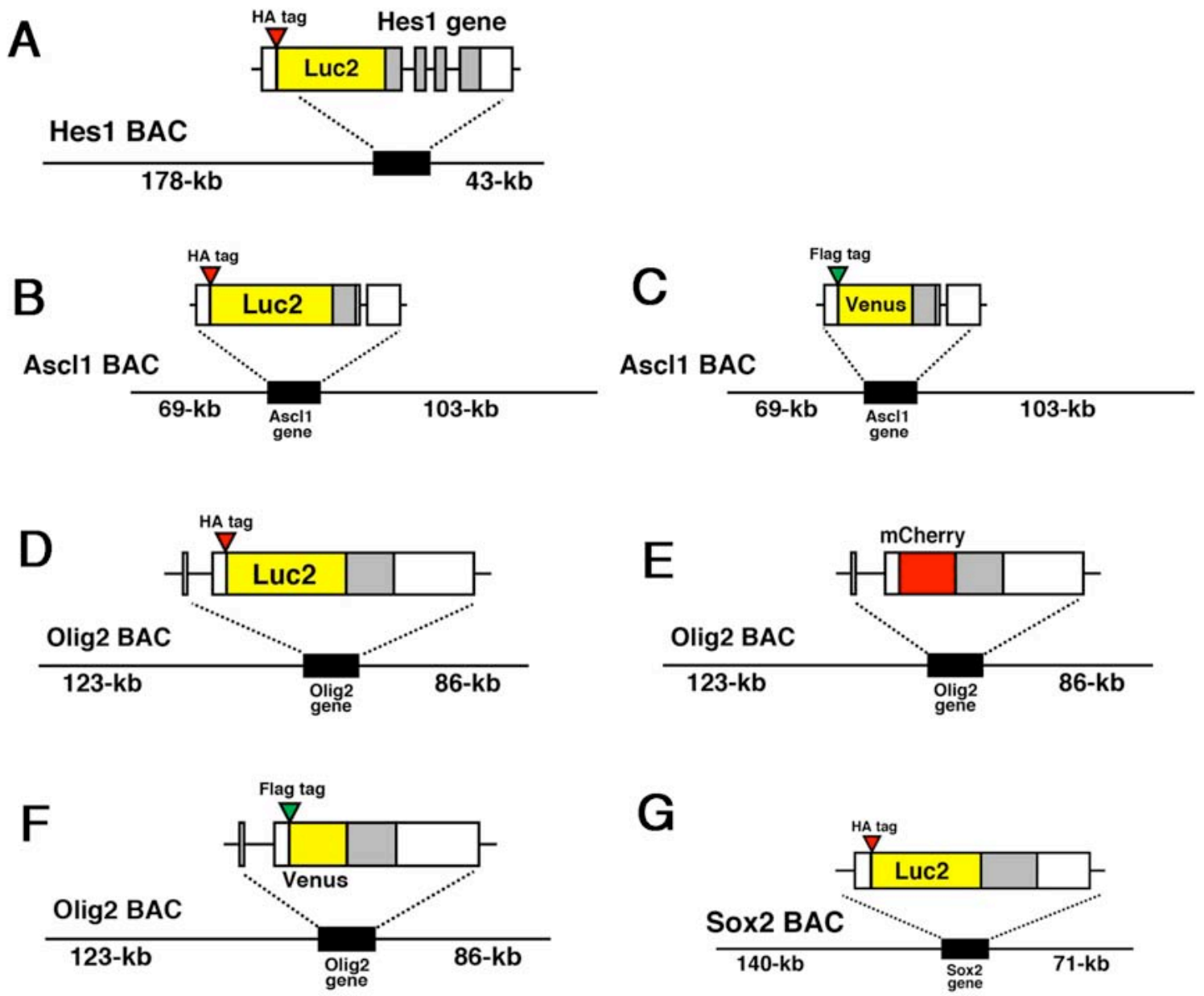

Fig. S1. Reporter-fusion BAC Tg constructs for monitoring protein expression dynamics of transcription factors.

(A) Luc2-Hes1 fusion BAC Tg mouse. (B) Luc2-Ascl1 fusion BAC Tg mouse. (C) Venus-Ascl1 fusion BAC Tg mouse. (D) Luc2-Olig2 fusion BAC Tg mouse. (E) mCherry-Olig2 fusion BAC Tg mouse. (F) Venus-Olig2 fusion BAC Tg mouse. (G) Luc2-Sox2 fusion BAC Tg mouse. Coding region is shown in grey. More detailed information is described in text and Supplementary Table S1. 

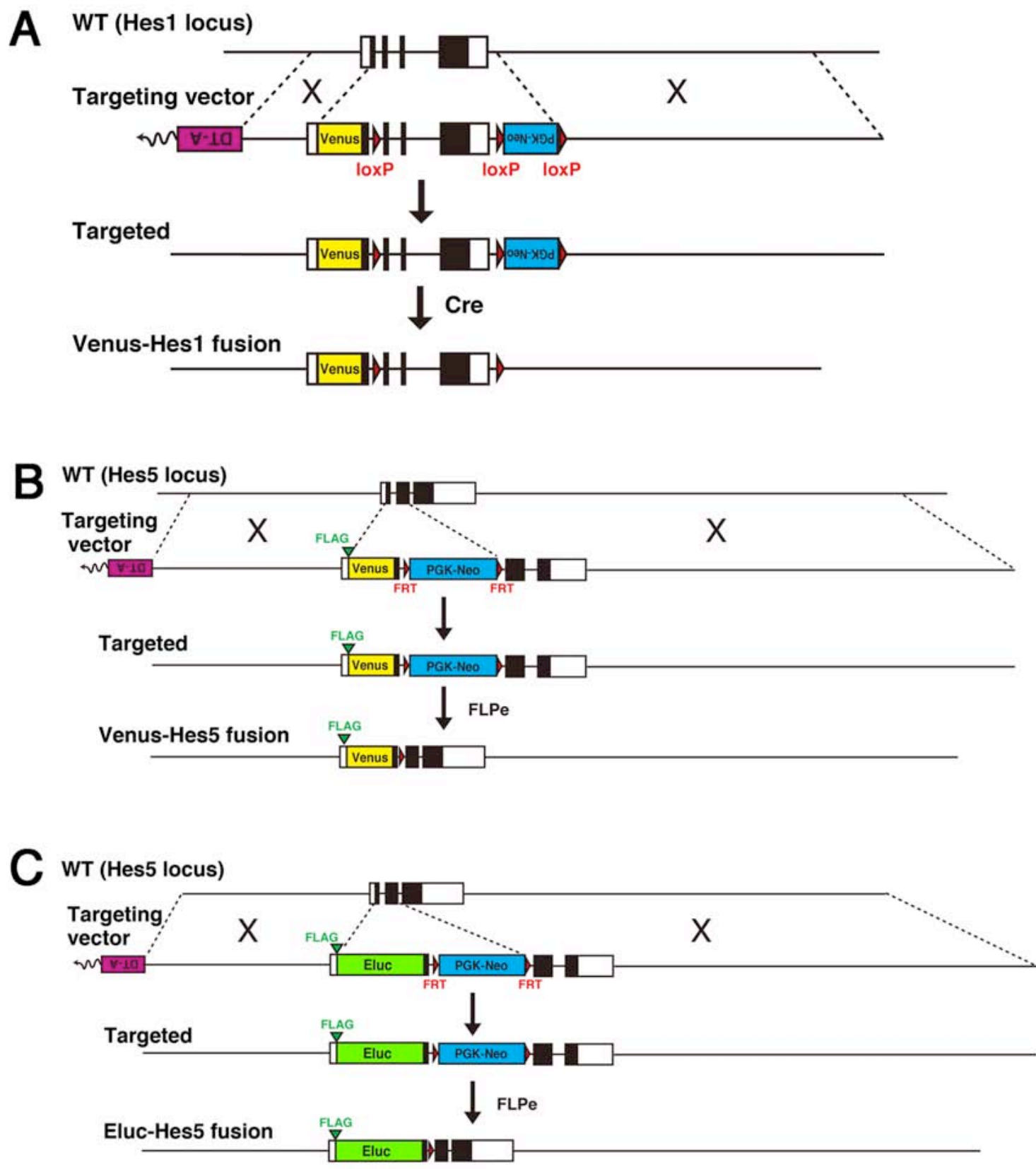

Fig. S2. Reporter-fusion knock-in strategy for monitoring expression dynamics of Hes1 and Hes5 proteins.

(A) Venus-Hes1 fusion knock-in mouse. (B) Venus-Hes5 fusion knock-in mouse. (C) ELuc-Hes5 fusion knock-in mouse. Closed box indicates coding region. More detailed information is described in text and Supplementary Table S1. 

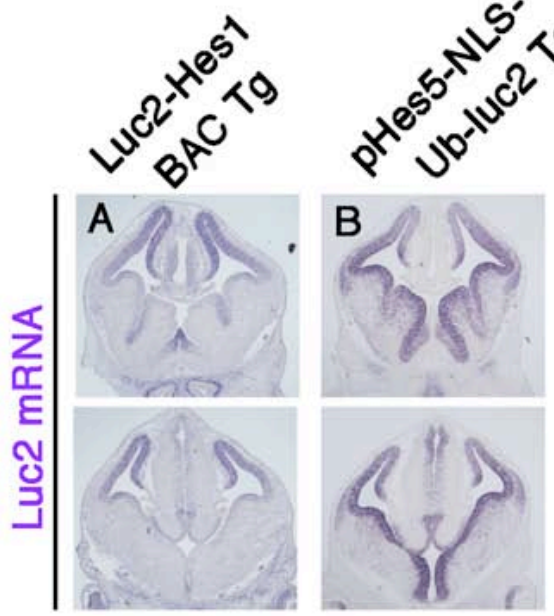

C
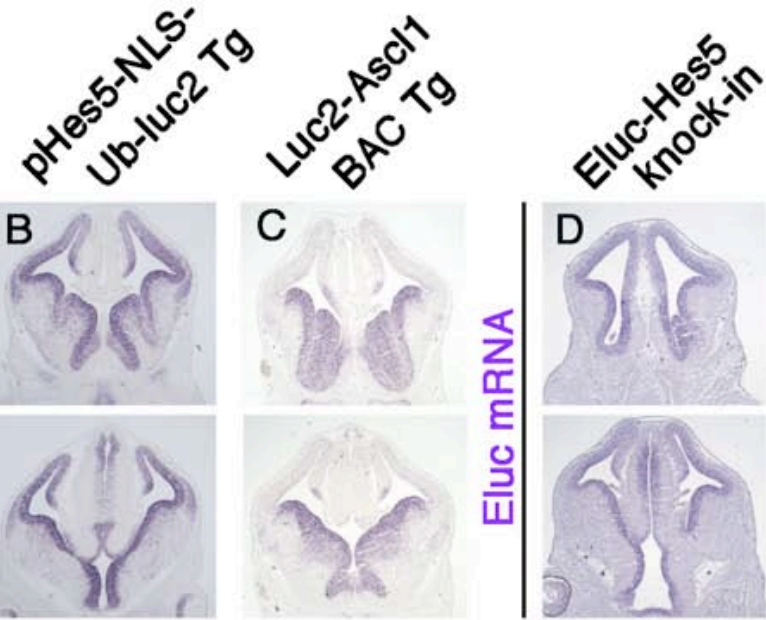

Venus-Hes1 knock-in

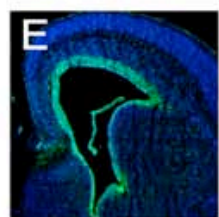

Venus-Hes1

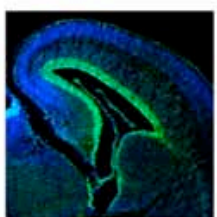

DAPI

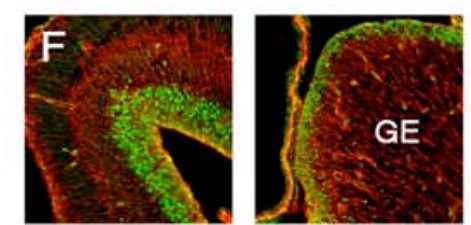

Venus-Hes1 Nestin

Venus-Hes5 knock-in

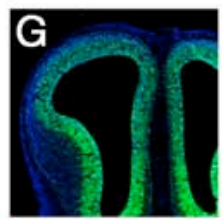

Venus-Hes5

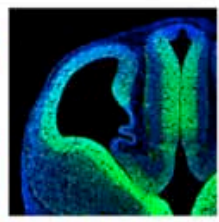

DAPI

Venus-Olig2 BAC Tg

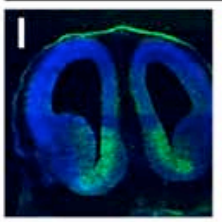

Venus-Olig2

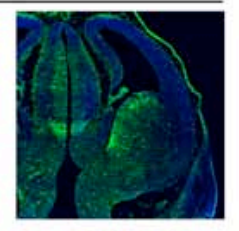

DAPI

Fig. S3. Faithful reporter expression in the reporter mice.

(A-D) In situ hybridization for Luc2 (A-C) and Eluc (D) mRNA of luciferase-reporter mice at E12.5. Coronal sections of the brains of Luc2-Hes1 fusion BAC Tg mouse (A), pHes5-NLS-Ub-Luc2-Tg mouse (B), Luc2-Ascl1 fusion BAC Tg mouse (C) and ELucHes5 fusion knock-in mouse (D). (E-J) Immunofluorescence for Venus (E-I) or mCherry $(\mathbf{J})$ of coronal sections of Venus-Hes1 fusion knock-in mouse $(\mathbf{F}, \mathbf{F})$, Venus-Hes5 fusion knock-in mouse $(\mathbf{G})$, Venus-Ascl1 fusion BAC Tg mouse $(\mathbf{H})$, Venus-Olig2 fusion BAC Tg mouse (I), and mCherry-Olig2 fusion BAC Tg mouse (J). Double immunolabelling for Nestin (red in $\mathbf{F}$ ) and Olig2 (green in $\mathbf{J}$ ) was performed. GE, Ganglionic eminence. 
A

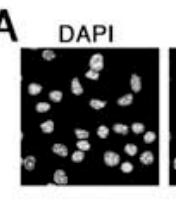

B
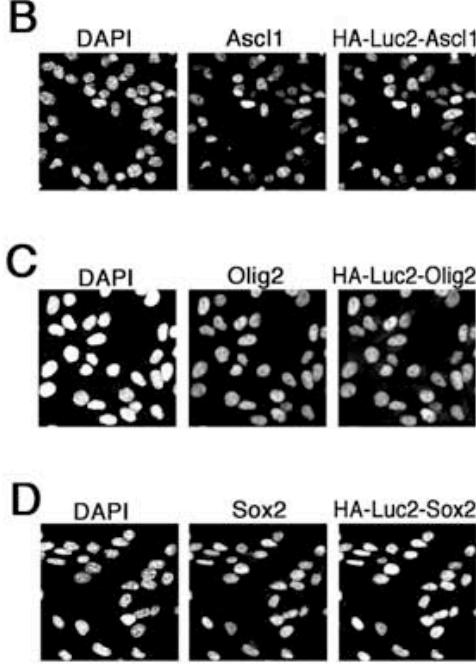

E
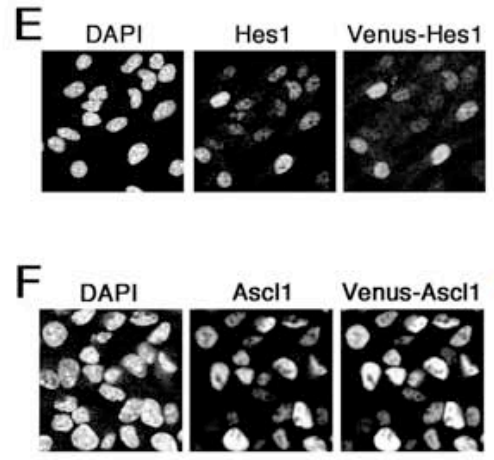

G
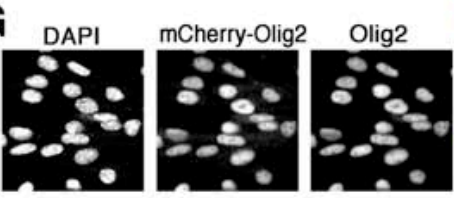

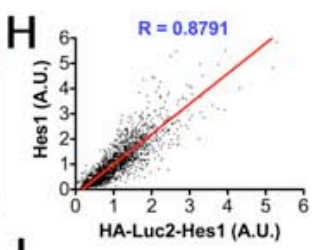

Ascl1
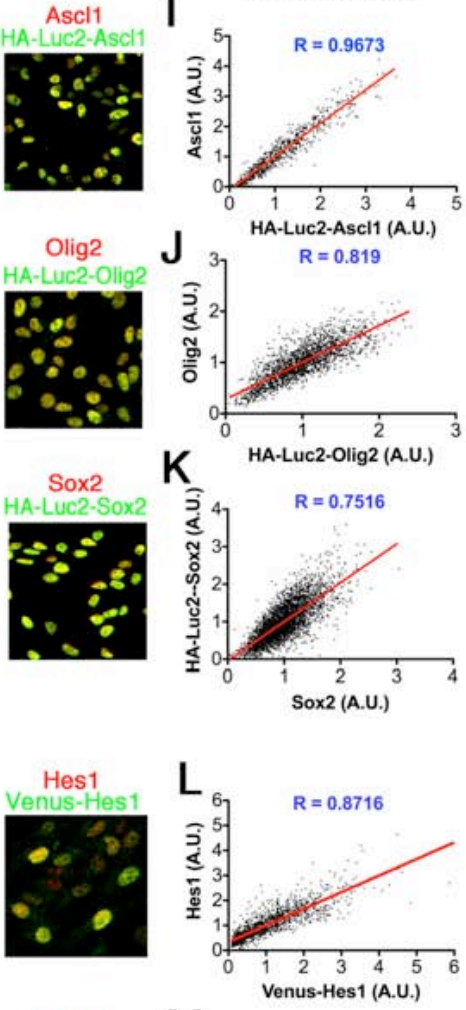

M
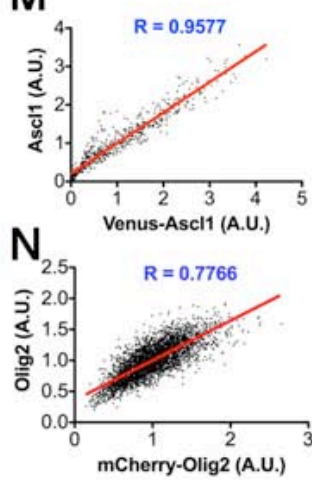

Fig. S4. Correlation between reporter and endogenous protein expression.

NPC cultures were prepared from reporter-fusion Tg or knock-in mice. (A-G)

Representative images of immunocytochemistry indicating well-correlated reporter protein expression to endogenous protein expression. (H) Scatter plot of Hes 1 protein levels versus HA-Luc2-Hes1 fusion protein levels. Each point represents Hes1 protein and HA-Luc2-Hes1 fusion protein signals in a single cell. (I) Asc11 versus HA-Luc2Asc11. (J) Olig2 versus HA-Luc2-Olig2. (K) Sox2 versus HA-Luc2-Sox2. (L) Hes1 versus Venus-Hes1. (M) Ascl1 versus Venus-Ascl1. (N) Olig2 versus mCherry-Olig2. Luciferase and fluorescent reporter expression correlated very well with endogenous protein expression in NPCs. 

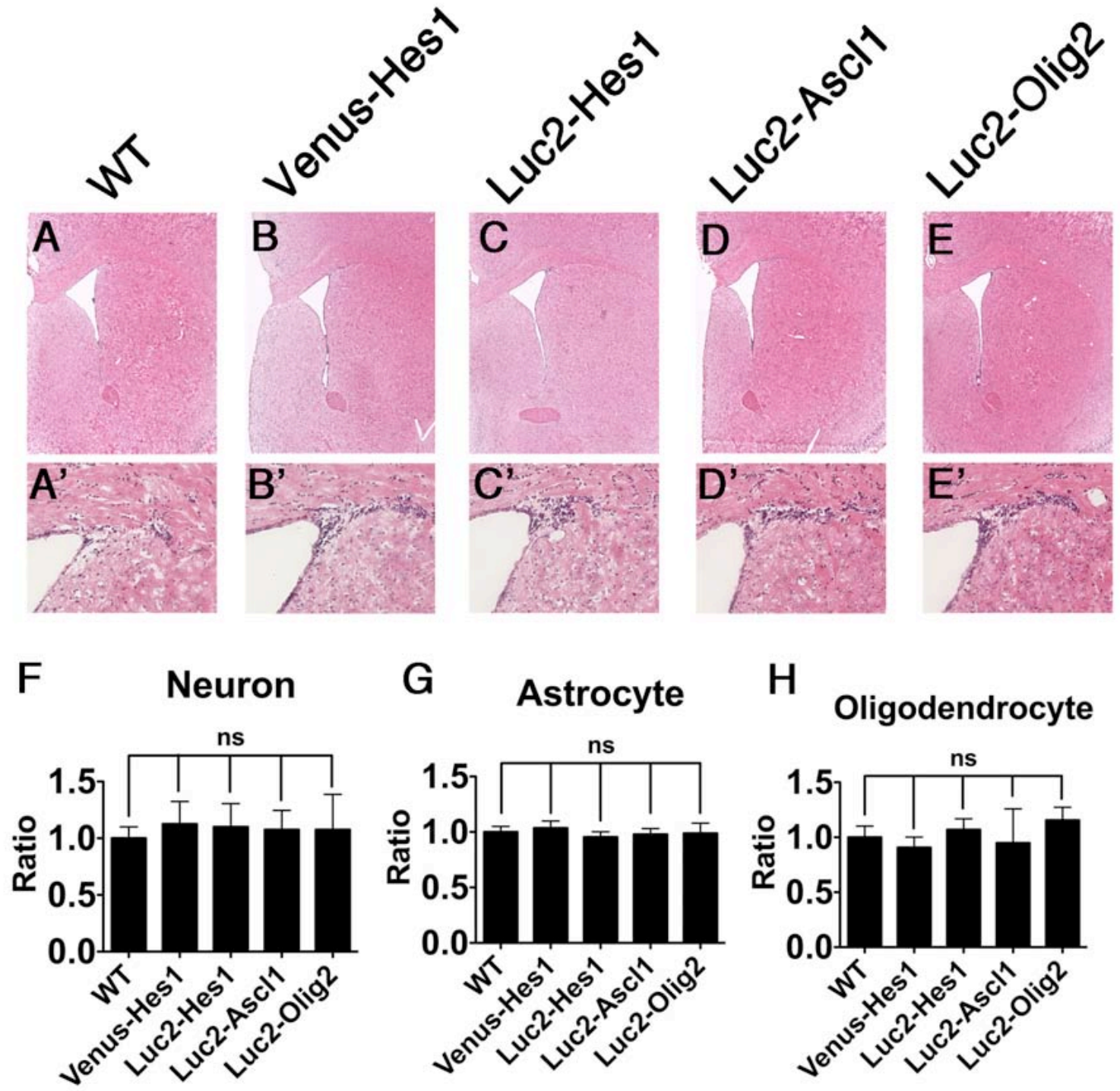

Fig. S5. Normal brain development of the reporter mice and their normal differentiation competency of NS cells.

(A-E') HE staining of the coronal sections of the adult brain from wild-type (A,A'), homozygous Venus-Hes1 fusion knock-in (B, $\mathbf{B}$ '), heterozygous Luc2-Hes1 fusion BAC $\operatorname{Tg}\left(\mathbf{C}, \mathbf{C}^{\prime}\right)$, heterozygous Luc2-Ascl1 fusion BAC Tg (D,D'), heterozygous Luc2-Olig2 fusion BAC Tg $\left(\mathbf{E}, \mathbf{E}^{\prime}\right)$ mice. The dorsolateral region of the subventricular zone of the lateral ventricle is enlarged in ( $\mathbf{A}^{\prime}-\mathbf{E}$ '). ( $\left.\mathbf{F}-\mathbf{H}\right)$ NS cells were generated from wild-type, homozygous Venus-Hes1 fusion knock-in, heterozygous Luc2-Hes1 fusion BAC Tg, heterozygous Luc2-Ascl1 fusion BAC Tg, and heterozygous Luc2-Olig2 fusion BAC Tg mice. These NS cells were transferred to a differentiation medium containing serum, and analyzed 7 days later by immunocytochemistry. Their competency to generate neurons, astrocytes, and oligodendrocytes was not distinguishable between all genotypes. ns: not significant, one-way ANOVA followed by Bonferroni post hoc test. 


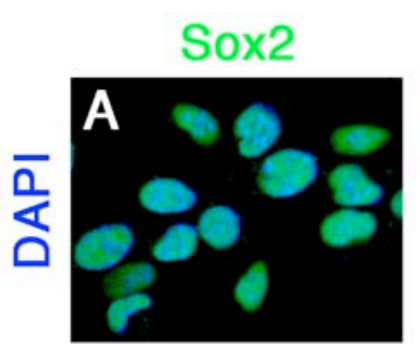

$\mathrm{E}$

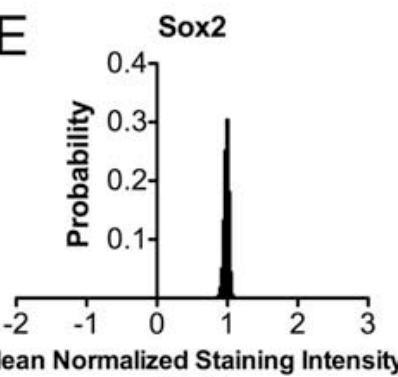

(A. U.)

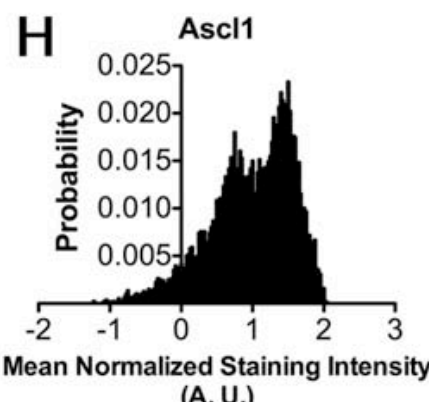

Hes1
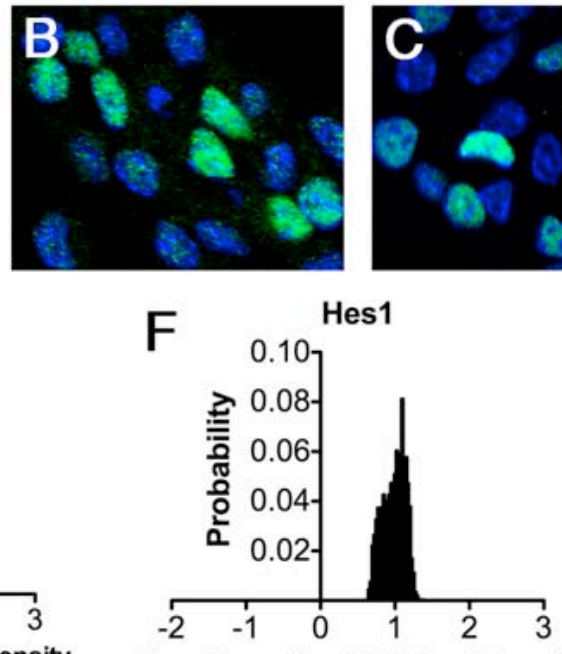

Mean Normalized Staining Intensity (A. U.)

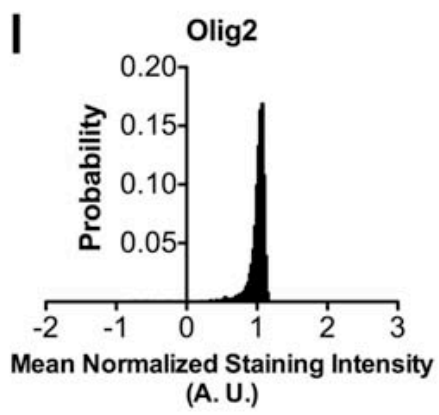

Ascl1
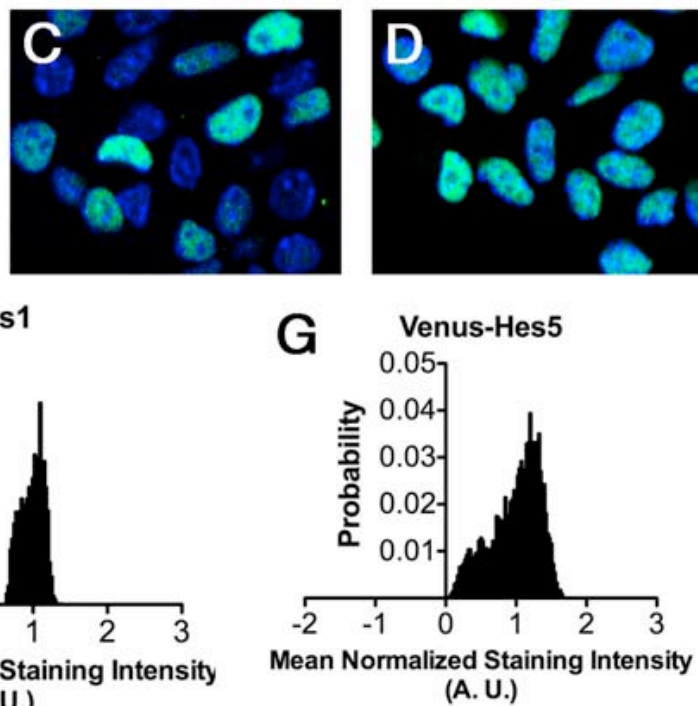

(A. U.)

J

Standard deviation (Average $=1.0)$

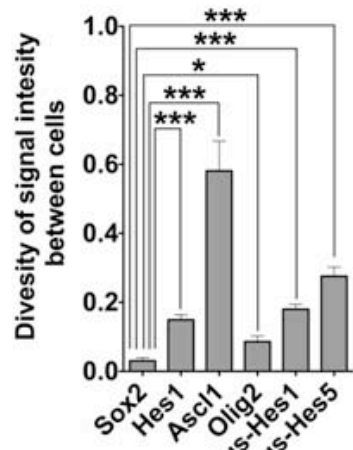

Fig. S6. Variable expression levels of bHLH factors in self-renewing NPCs.

(A-D) Proliferating NPCs were immunostained with anti-Sox2 (A), anti-Hes1 (B), antiAscl1 (C), and anti-Olig2 (D) antibodies. Hes1, Ascl1, and Olig2 expression levels were variable while another NPC-specific factor, Sox 2 , was expressed at a relatively constant level. (E-I) Histograms of mean normalized Sox2, Hes1, Venus-Hes5, Ascl1, and Olig2 staining intensity in proliferating NPCs. (J) Comparison of the standard deviations of the expression levels. The variability of Sox 2 expression was very small compared to the other factors. ${ }^{*} p<0.05,{ }^{* * *} p<0.001$; two-tailed Student's $t$-test. 
NS cells

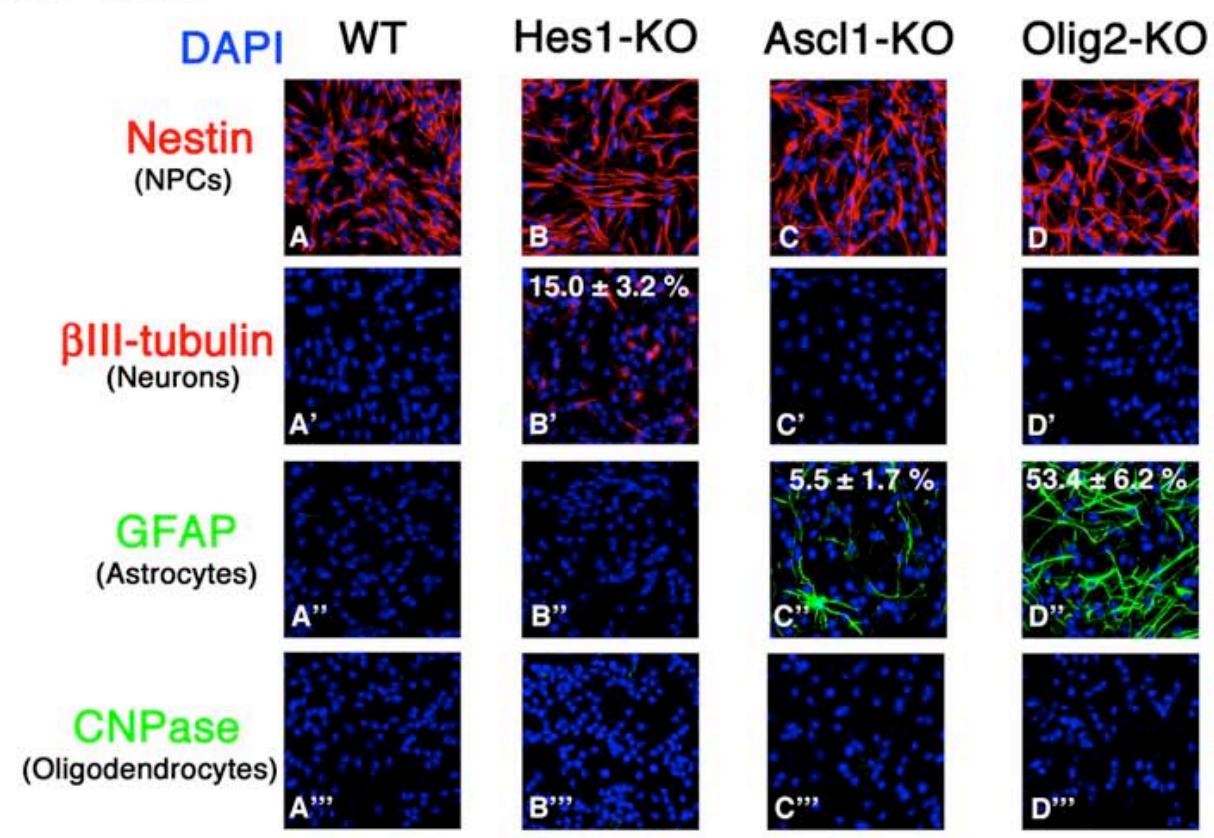

Differentiated cells from NS cells
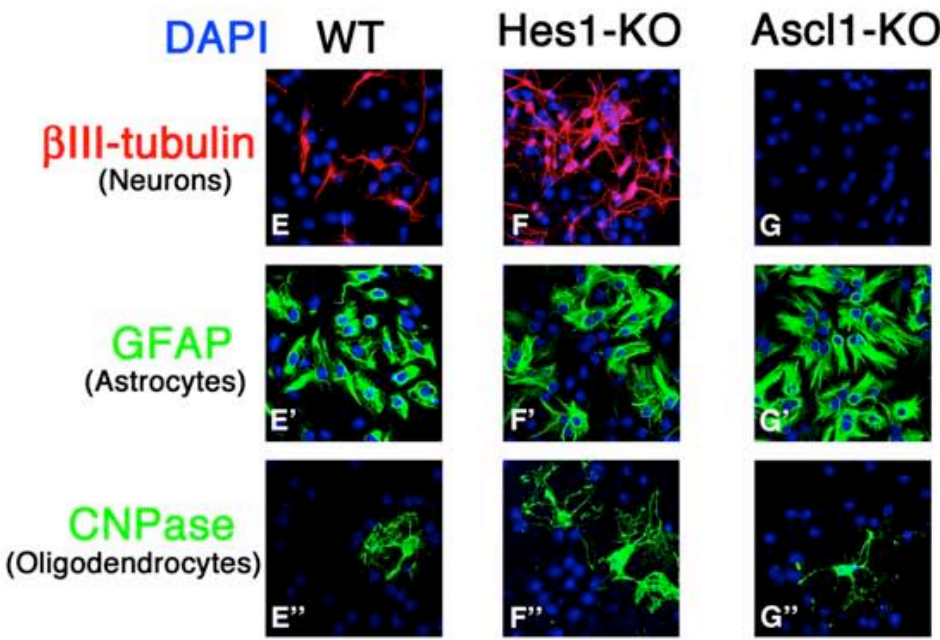

Olig2-KO
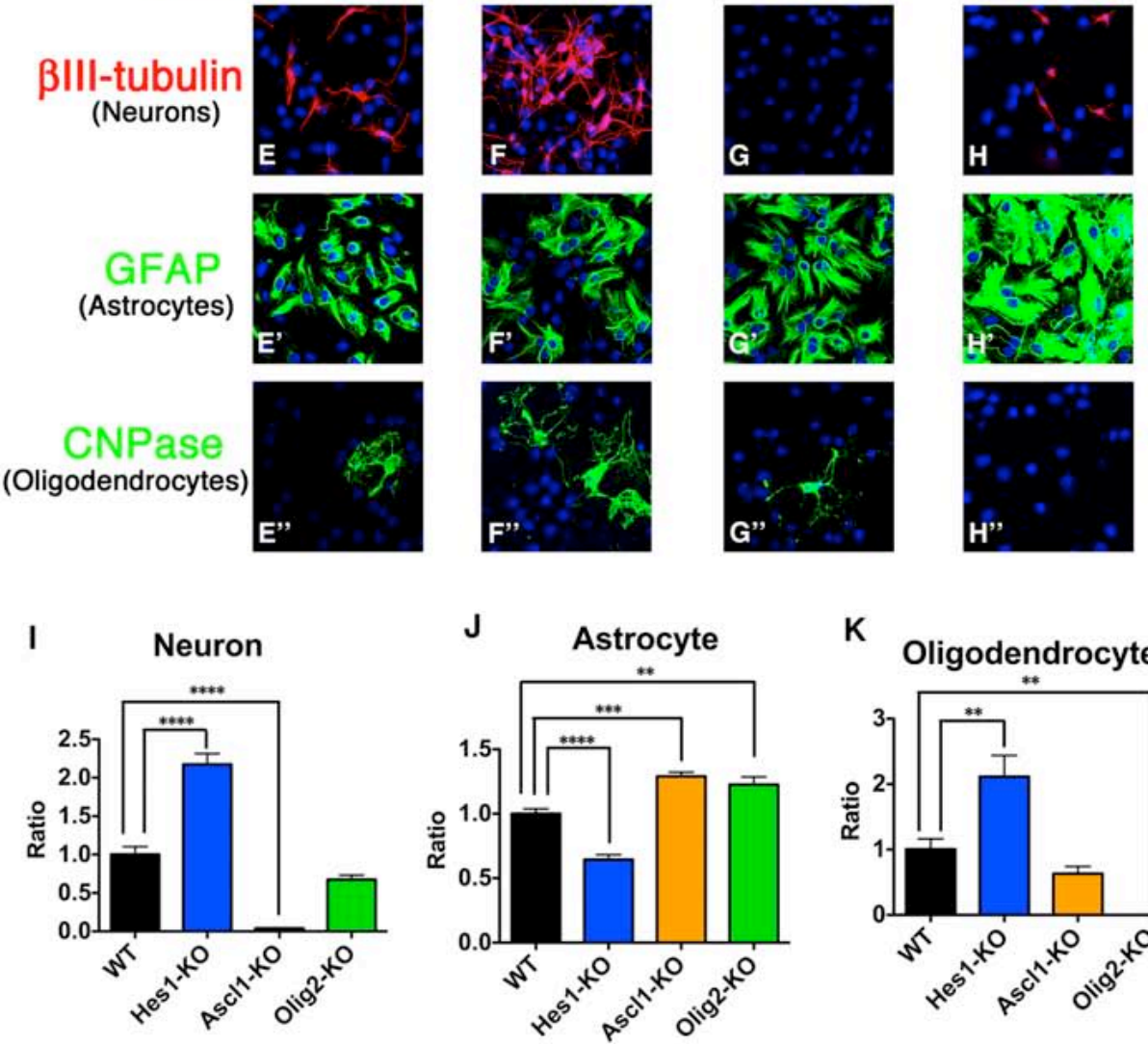

K Oligodendrocyte

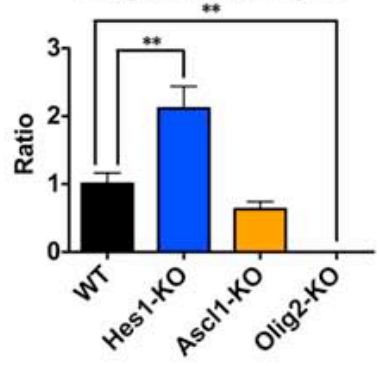


Fig. S7. Abnormal differentiation competency of NS cells mutant for bHLH factors. NS cells were generated from wild type (A-A'"'), Hes1-KO (B-B','), Ascl1-KO (C-C','), and Olig2-KO (D-D'"') mice. These NS cells were immunostained with anti-Nestin (NPC marker), anti- $\beta$ III-tubulin (neuron marker), anti-GFAP (astrocyte marker), and antiCNPase (oligodendrocyte marker) antibodies. $15 \%$ of Hes $1-\mathrm{KO}$ cells expressed $\beta$ IIItubulin, and 5.5\% of Ascl1-KO cells and 53\% of Olig2-KO cells displayed GFAP expression before induction of differentiation. (E-K) These NS cells were transferred to a differentiation medium containing serum, and analyzed 7 days later by immunocytochemistry. Their competency to generate neurons, astrocytes, and oligodendrocytes was significantly reduced in the absence of Ascl1, Hes1, and Olig2, respectively. $* p<0.05, * * p<0.01, * * * p<0.001, * * * * p<0.0001$, one-way ANOVA followed by Bonferroni post hoc test. 


\section{Luminescence}
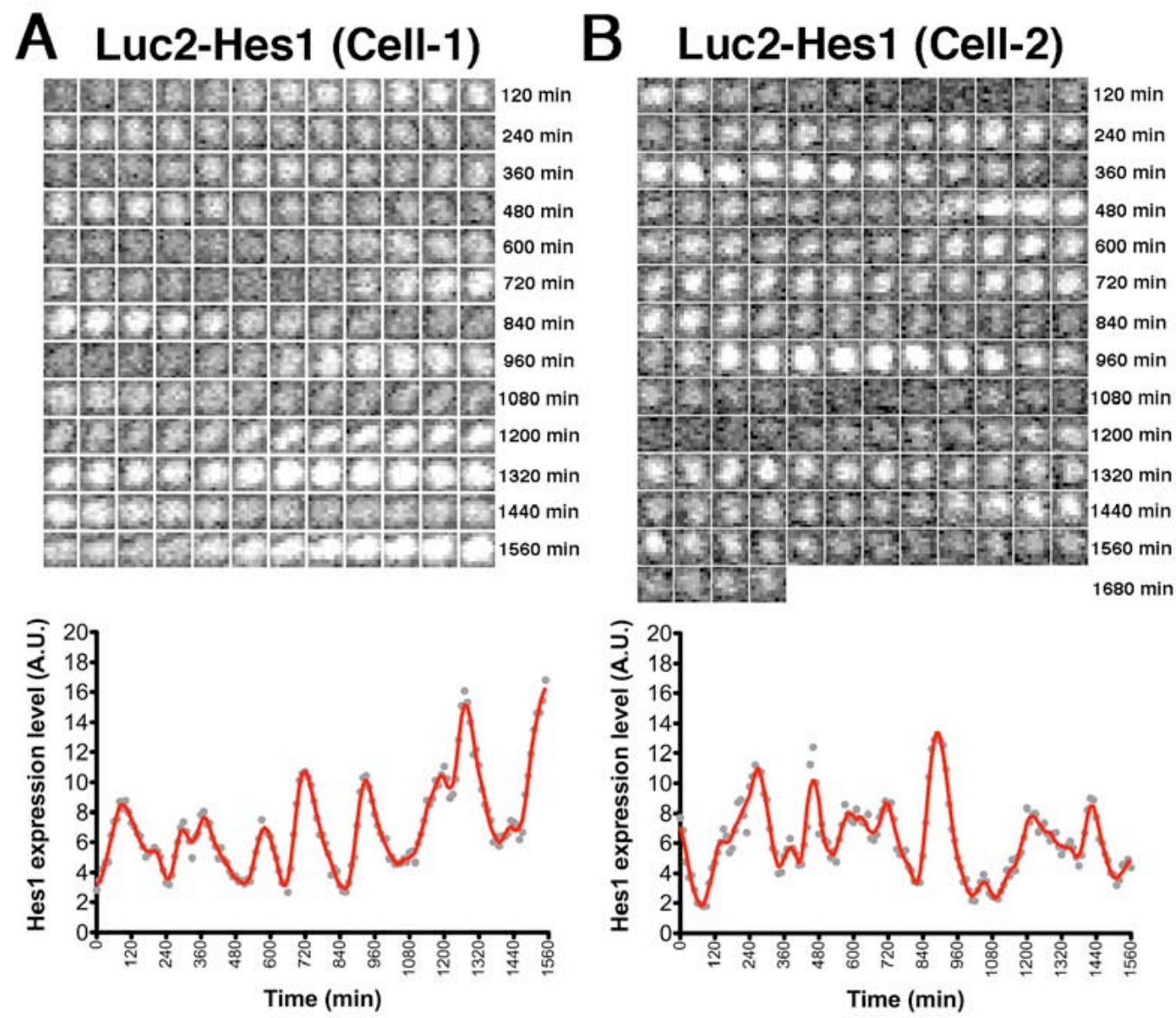

\section{Fluorescence}
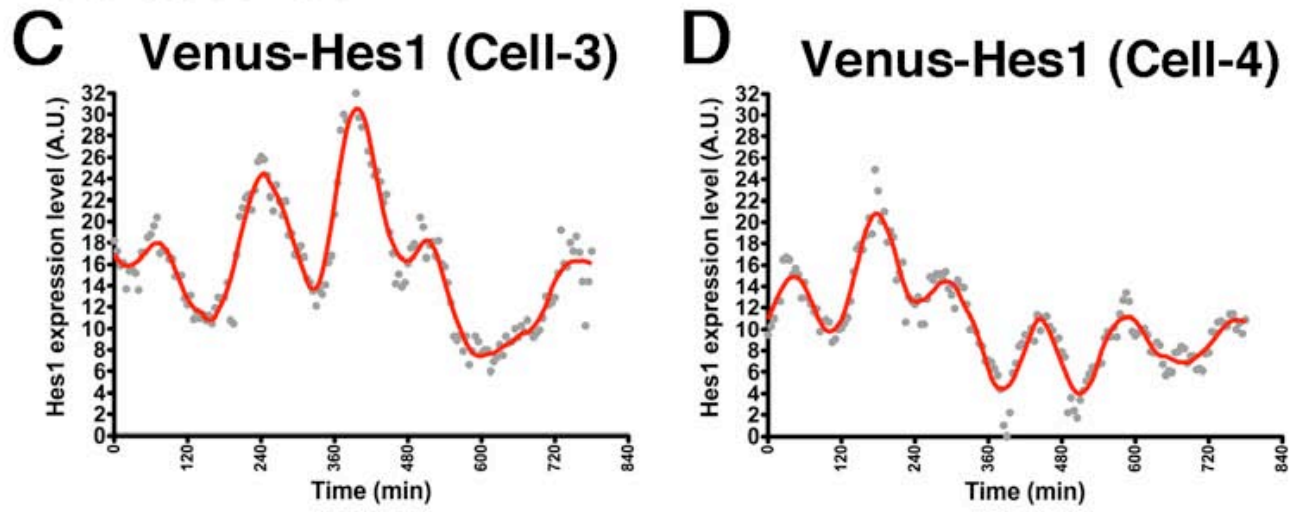

Fig. S8. Oscillatory expression of Hes1 protein in NPCs.

NPC cultures were prepared from Luc2-Hes1 fusion BAC Tg mice $(\mathbf{A}, \mathbf{B})$ or Venus-Hes1 fusion knock-in mice $(\mathbf{C}, \mathbf{D})$. $(\mathbf{A}, \mathbf{B})$ Bioluminescence images and quantification of Luc2Hes1 fusion protein dynamics in self-renewing NPCs. (C,D) Quantification of VenusHes 1 fusion protein dynamics in self-renewing NPCs. 
Luminescence
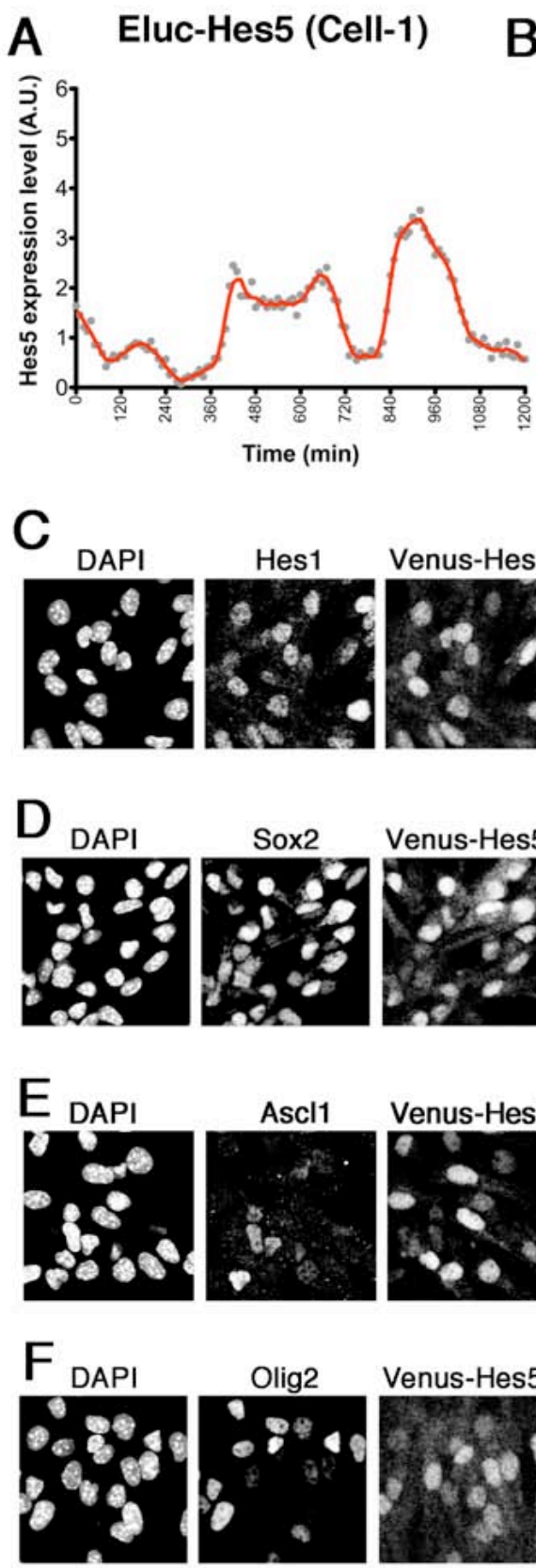

Hes1

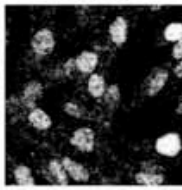

Sox2

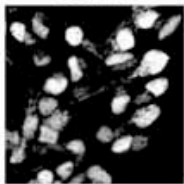

Ascl1
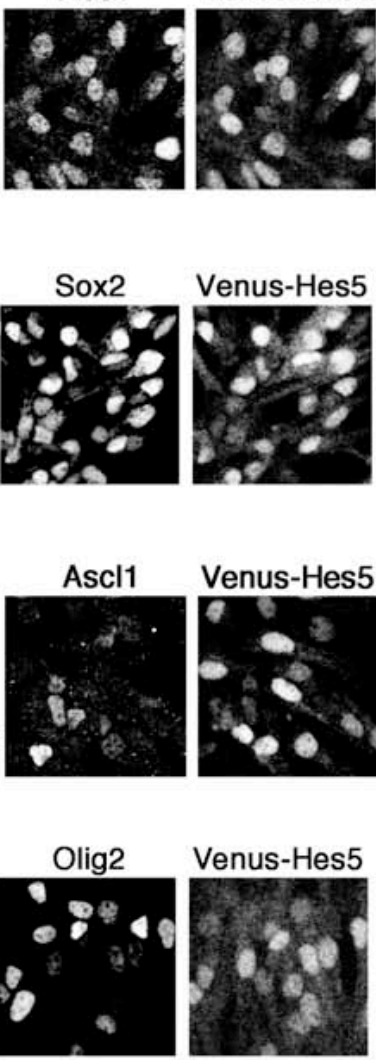

\section{Fluorescence}
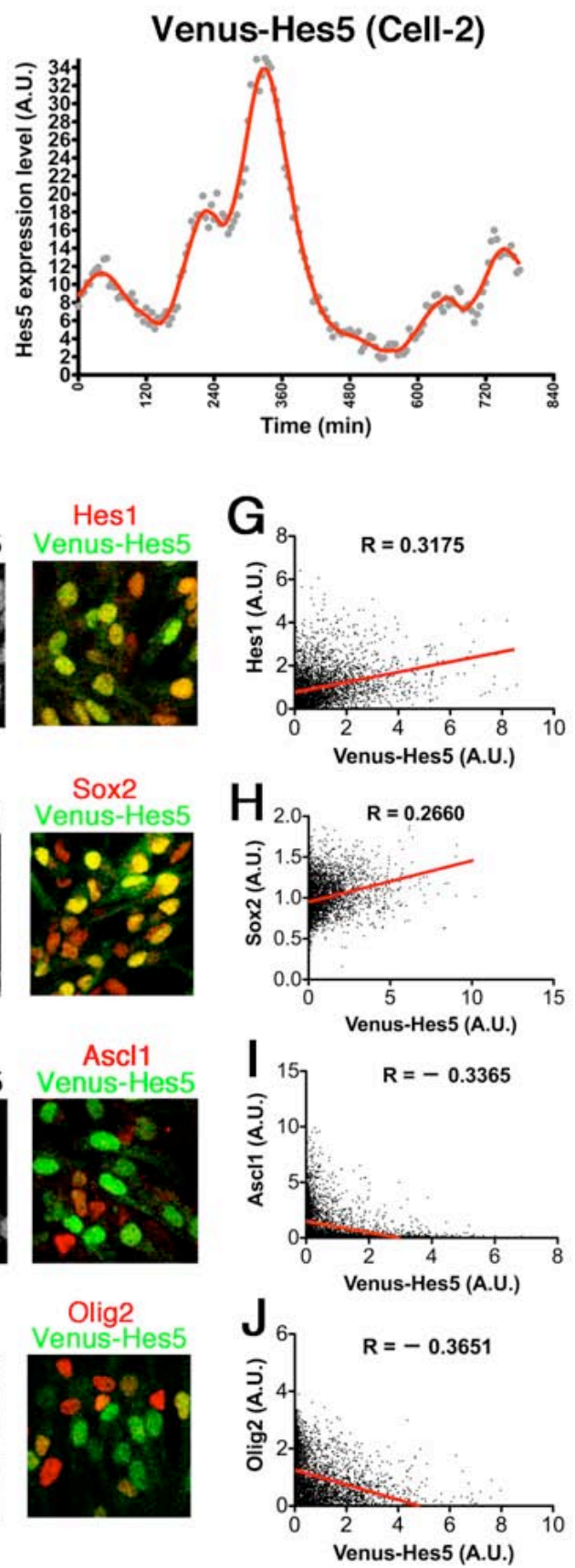

Fig. S9. Oscillatory expression of Hes5 protein in NPCs.

NPC cultures were prepared from Eluc-Hes5 fusion knock-in mice (A) or Venus-Hes5 fusion knock-in mice (B). (A) Quantification of ELuc-Hes5 fusion protein dynamics in self-renewing NPCs. (B) Quantification of Venus-Hes5 fusion protein dynamics in selfrenewing NPCs. (C,G) Representative images of immunocytochemistry, and scatter plot of Hes1 protein levels versus Venus-Hes5 fusion protein levels. Each point represents Hes 1 protein and Venus-Hes5 fusion protein signals in a single cell. (D,H) Sox2 versus Venus-Hes5. (E,I) Ascl1 versus Venus-Hes5. (F,J) Olig2 versus Venus-Hes5. 

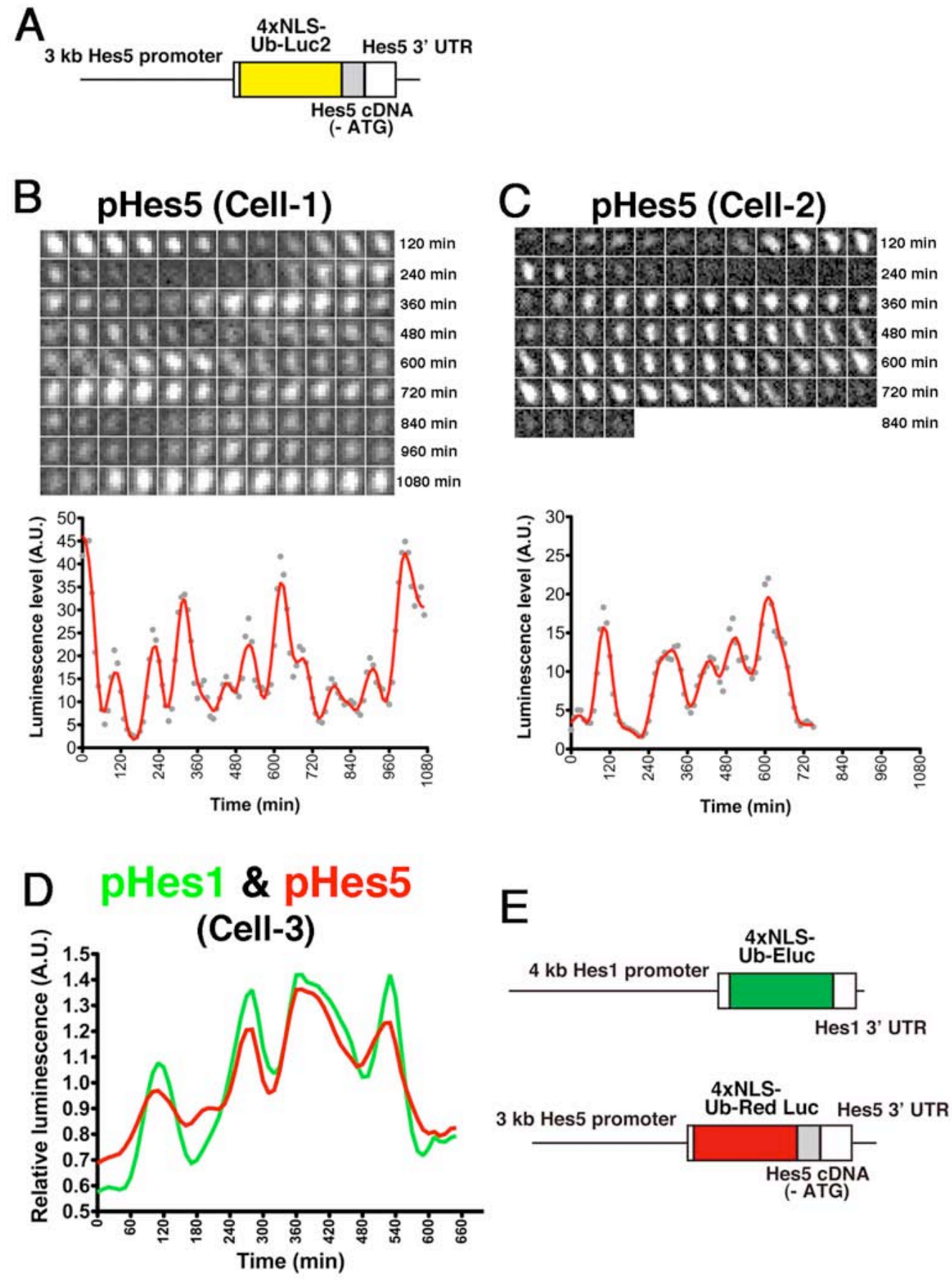

Fig. S10. Oscillatory expression of the Hes5 promoter activity in NPCs.

(A) The structure of pHes5-NLS-Ub-luc2 that monitors the Hes5 promoter activity. (B,C) NPC cultures were prepared from pHes5-NLS-Ub-luc2 Tg mice. Bioluminescence images and quantification of the Hes5 promoter activity in self-renewing NPCs. (D) Quantification of the Hes1 promoter and Hes5 promoter activity in a single cell. Hes 1 and Hes 5 oscillations occurred in phase. (E) Reporter constructs for two-color simultaneous imaging of two promoter activities in single cells. These pHes1- and pHes5-reporter constructs were co-transfected to NPCs by nucleofection. 
Luminescence
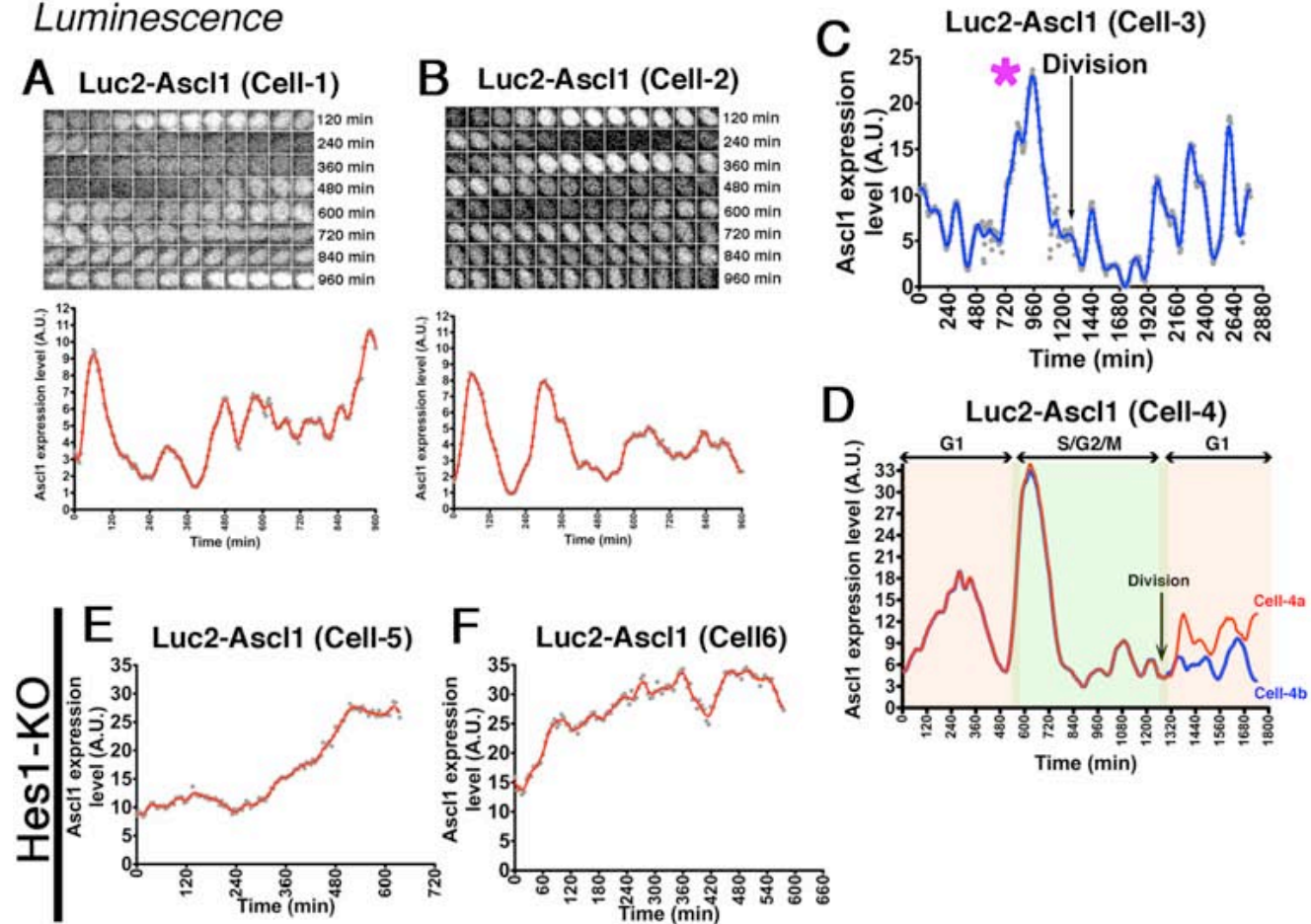

Fluorescence

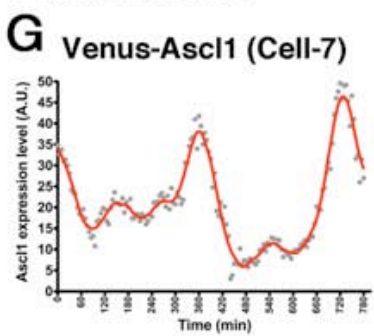

H Venus-Ascl1 (Cell-8)
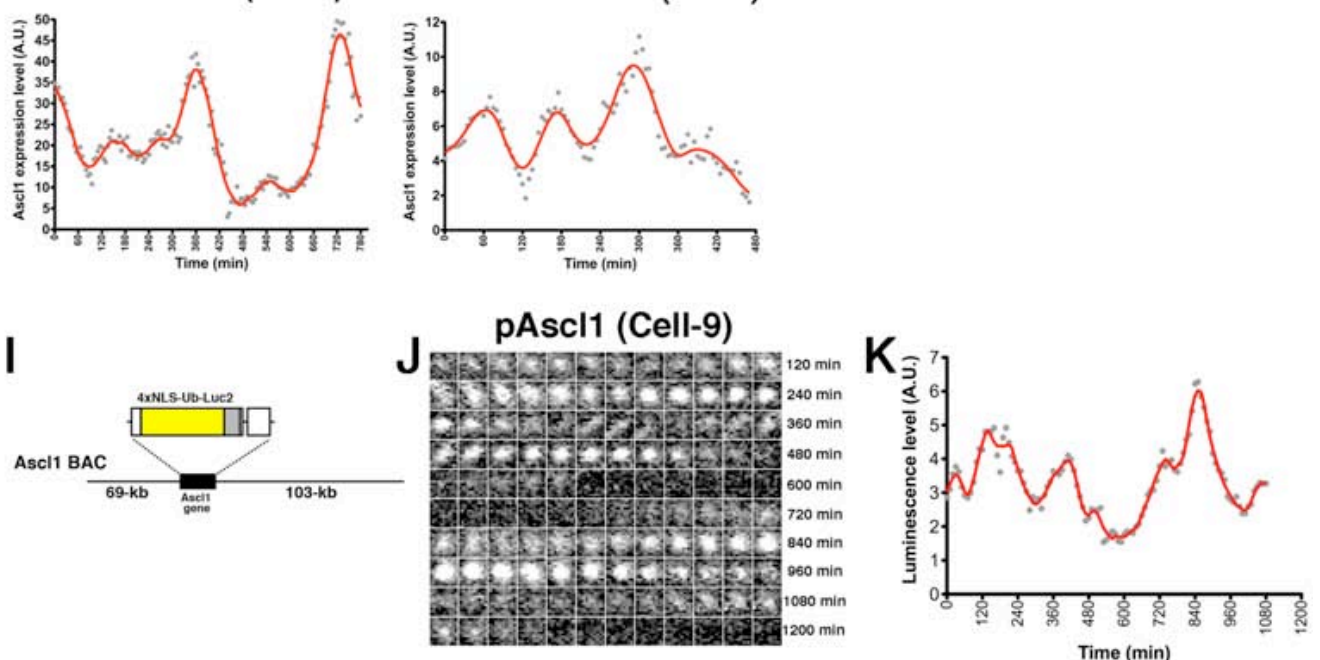

Fig. S11. Oscillatory expression of Ascl1 protein and promoter activity in NPCs. NPC cultures were prepared from Luc2-Ascl1 fusion BAC Tg mice (A-F), Venus-Ascl1 fusion BAC Tg mice (G,H), and Ascl1-NLS-Ub-luc2 BAC Tg mice (I-K). (A-D) Bioluminescence images and quantification of Luc2-Ascl1 fusion protein dynamics in self-renewing NPCs. Cell cycle phase progression was monitored by the Fucci probe in Cell-3 (D). Bioluminescence images and quantification of the Luc2-Ascl1 fusion protein dynamics in Hes1-KO NPCs $(\mathbf{E}, \mathbf{F})$. $(\mathbf{G}, \mathbf{H})$ Quantification of Venus-Ascl1 fusion protein dynamics in self-renewing NPCs. (I-K) Transgenic construct of Ascl1-NLS-Ub-luc2 $\mathrm{BAC} \mathrm{Tg}$ mice (I). Bioluminescence images and quantification of the Ascll promoter activity in self-renewing NPCs $(\mathbf{J}, \mathbf{K})$. 


\section{Luminescence}
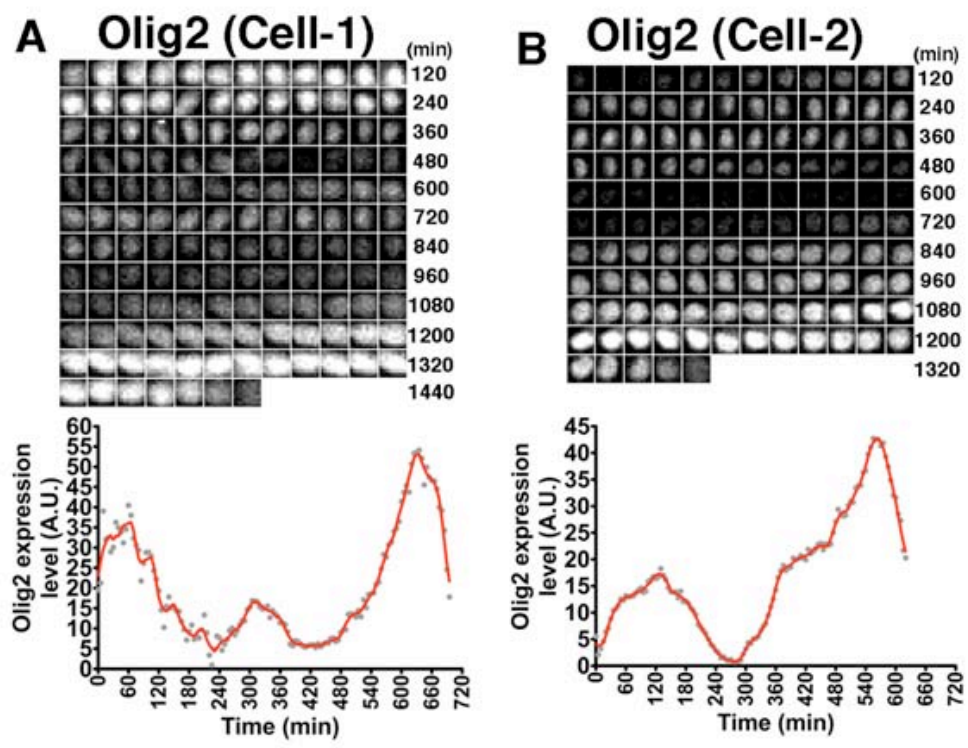

C Olig2 (Cell-3)

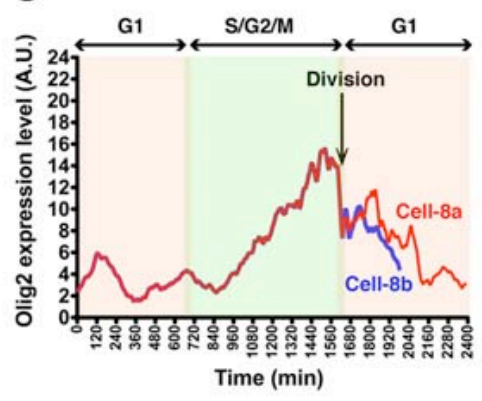

\section{Fluorescence}
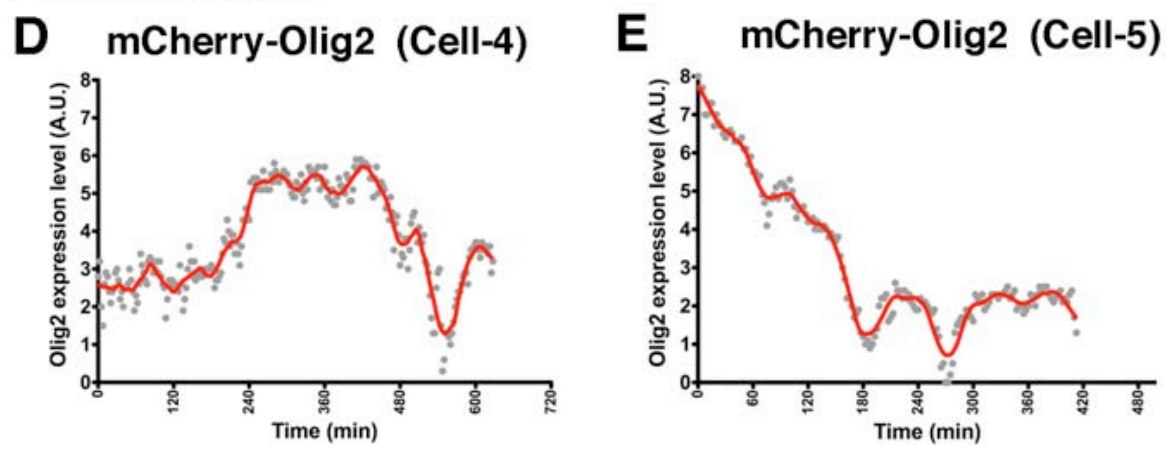

Fig. S12. Oscillatory expression of Olig2 protein in NPCs.

NPC cultures were prepared from Luc2-Olig2 fusion BAC Tg mice (A-C) and mCherryOlig2 fusion BAC Tg mice (D,E). (A-C) Bioluminescence images and quantification of Luc2-Olig2 fusion protein dynamics in self-renewing NPCs. Cell-cycle phase progression was monitored by the Fucci probe in Cell-3 (C). (D,E) Quantification of mCherry-Olig2 fusion protein dynamics in self-renewing NPCs. 


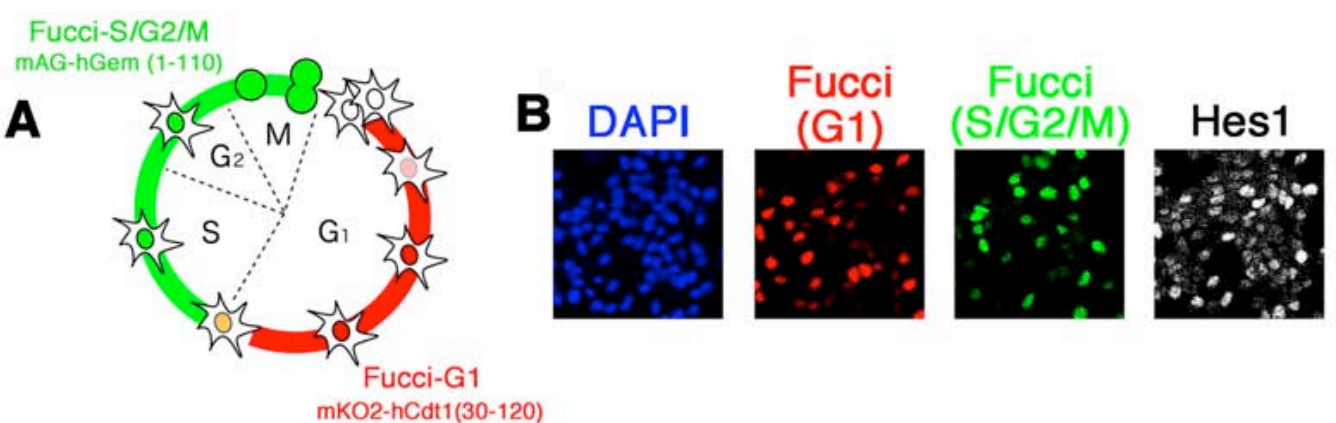

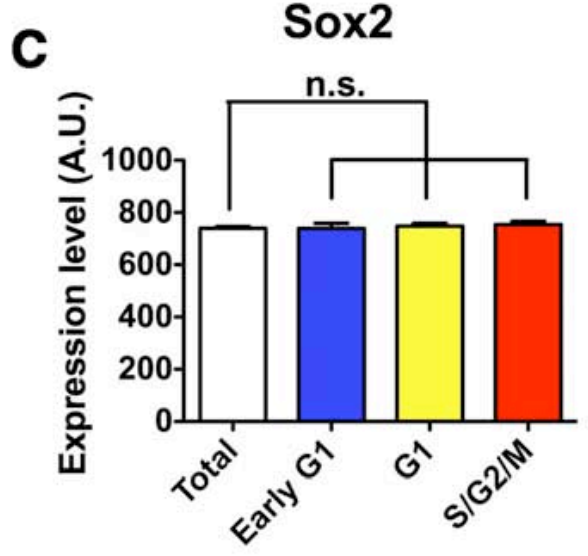

Cell cycle phase

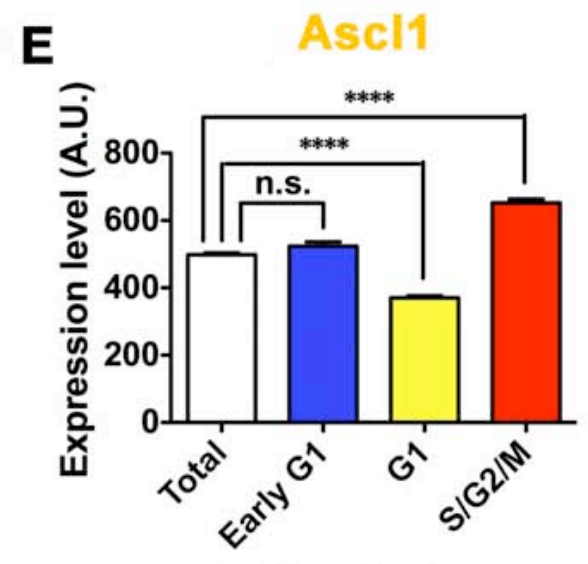

Cell cycle phase

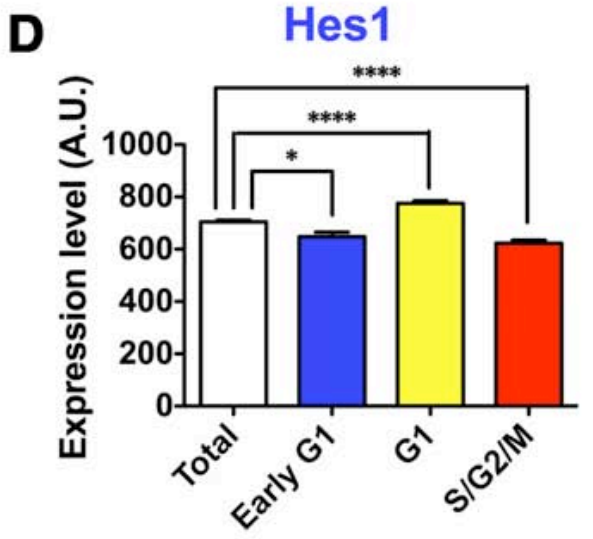

Cell cycle phase

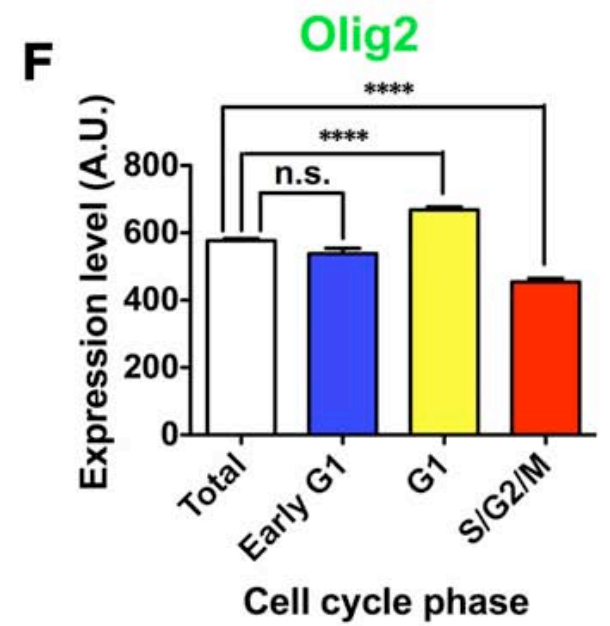

Fig. S13. Comparison of average expression levels of transcription factors in different cell-cycle phases of self-renewing NPCs.

(A) Fucci probe that labels G1 phase nuclei in red and S/G2/M phase nuclei in green. NPC cultures were prepared from pCAG-mAG-hGem(1/110) and pCAG-mKO2hCdt 1(30/120) double-transgenic mice. (B) Representative images of immunocytochemistry for analyzing expression levels of Hes1 with cell-cycle phase information in individual cells. (C-F) Comparison of average expression levels of Sox2 (C), Hes1 (D), Ascl1 (E), and Olig2 (F) in different cell-cycle phases of self-renewing NPCs. $* p<0.05, * * p<0.01, * * * p<0.001, * * * * p<0.0001$, one-way ANOVA followed by Bonferroni post hoc test. 

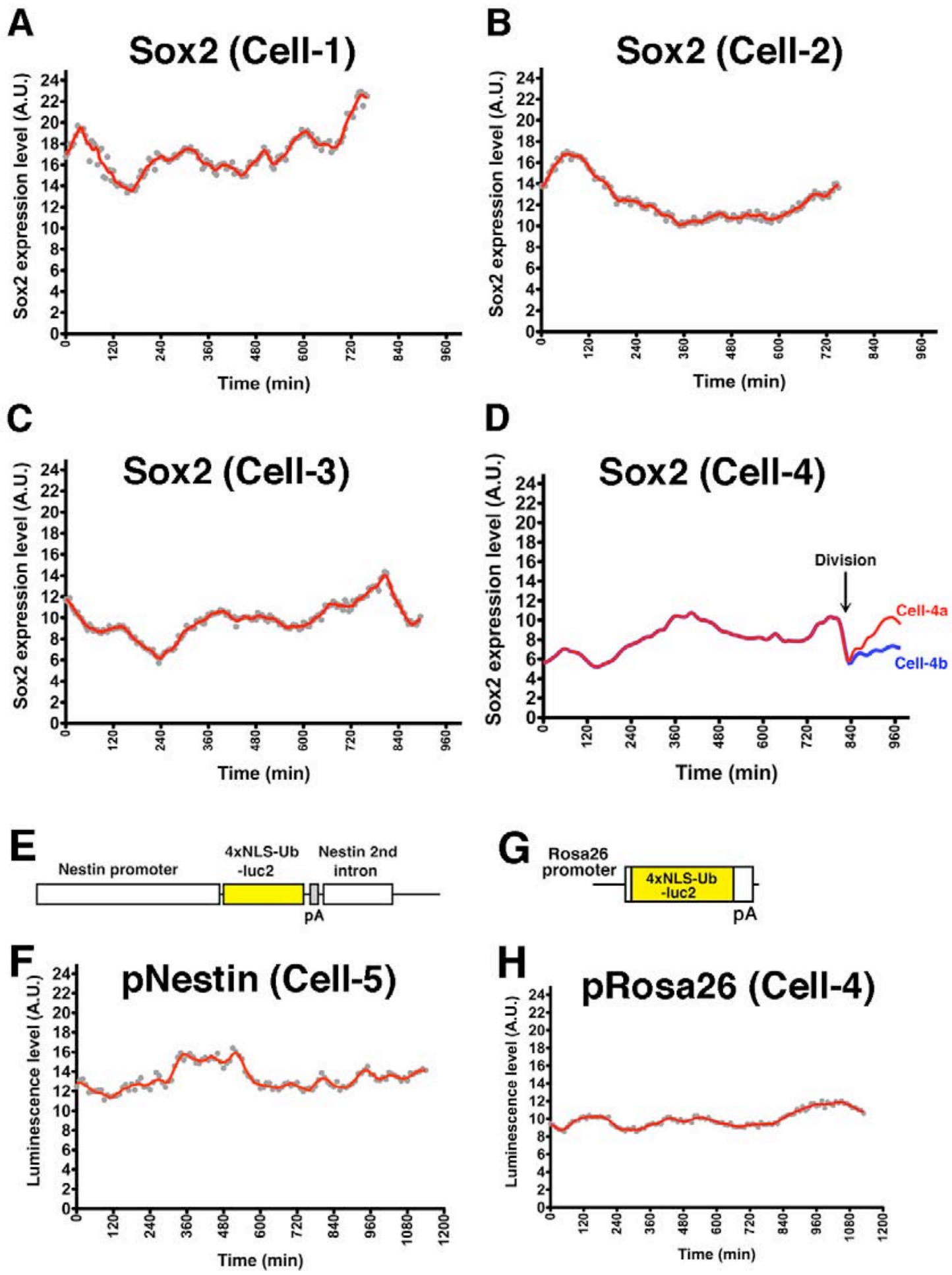

Fig. S14. Stable Sox 2 protein expression and promoter activity of Nestin and Rosa26. (A-D) NPC cultures were prepared from Luc2-Sox2 fusion BAC Tg mice. Quantification of Luc2-Sox 2 fusion protein dynamics in self-renewing NPCs. (E,G) Reporter constructs for imaging the Nestin and Rosa26 promoter activities. (F) Quantification of the Nestin promoter activity in NPCs. (H) Quantification of the Rosa26 promoter activity in NPCs. Reporter constructs were transfected to NPCs by nucleofection. 
A
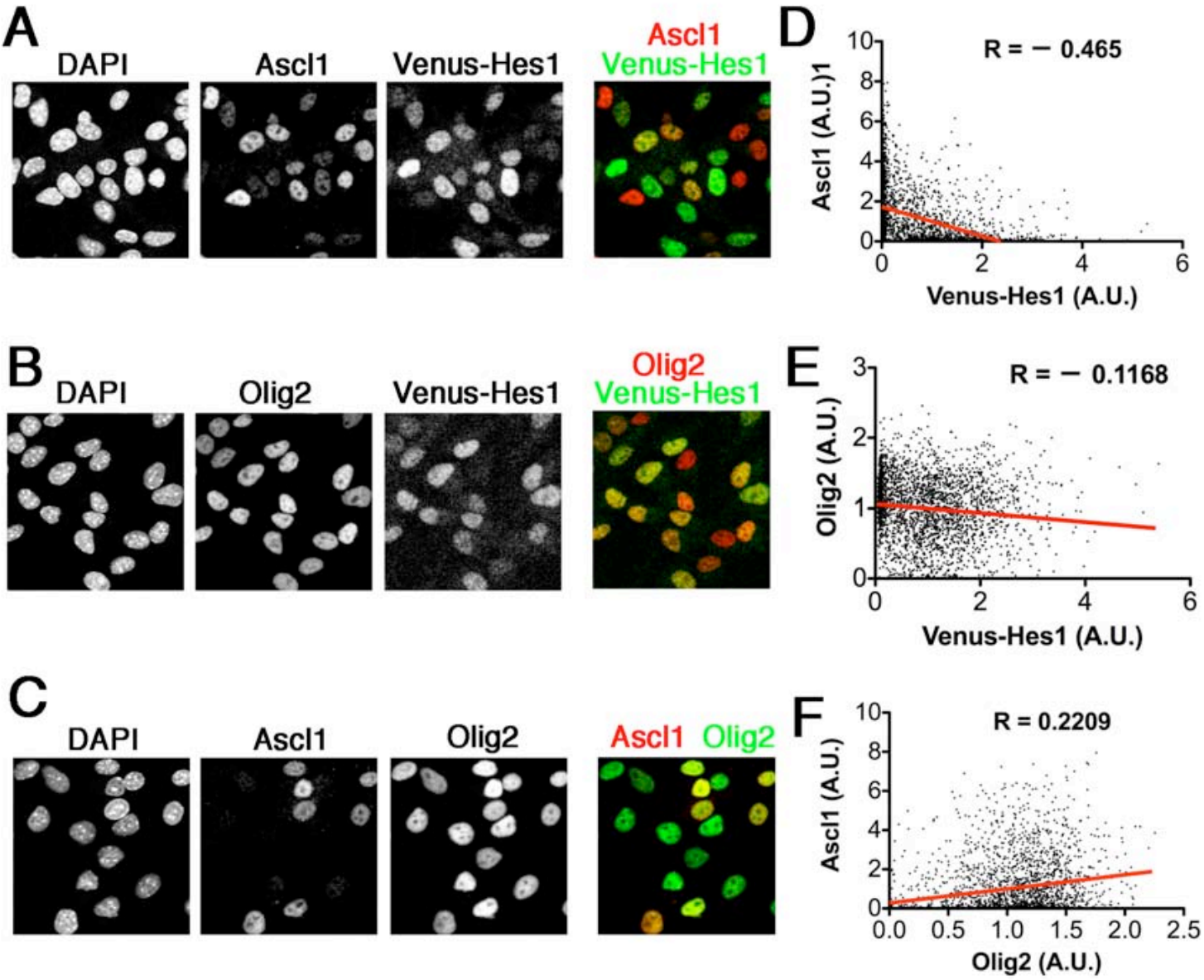

Fig. S15. Correlation between bHLH protein expression in self-renewing NPCs. (A-C) Representative images of immunocytochemistry of NPCs. (D) Scatter plot of Ascl1 protein levels versus Venus-Hes1 fusion protein levels. Each point represents Ascl1 protein and Venus-Hes1 fusion protein signals in a single cell. (E) Olig2 versus Venus-Hes1. (F) Ascl1 versus Olig2. 

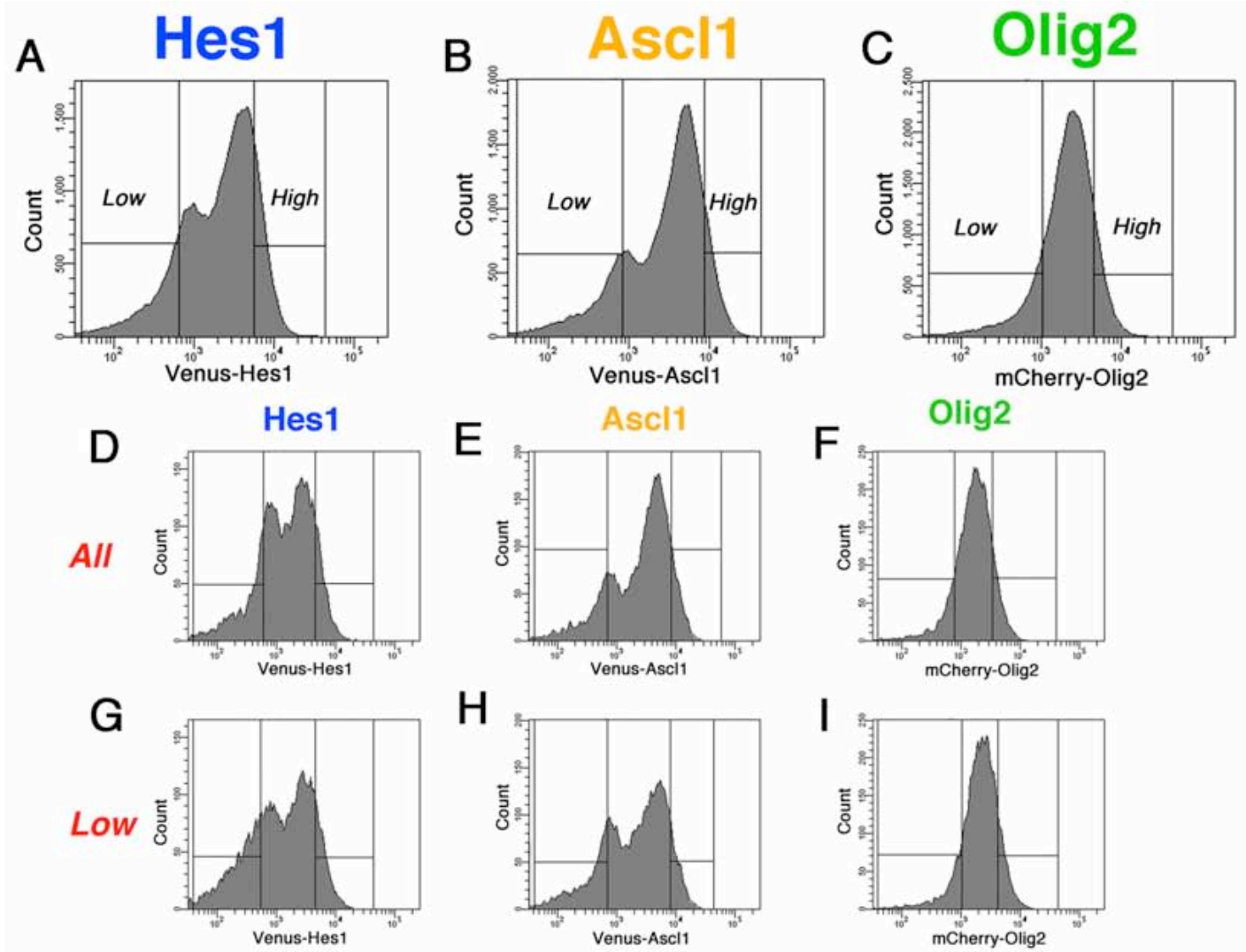

$\mathrm{H}$
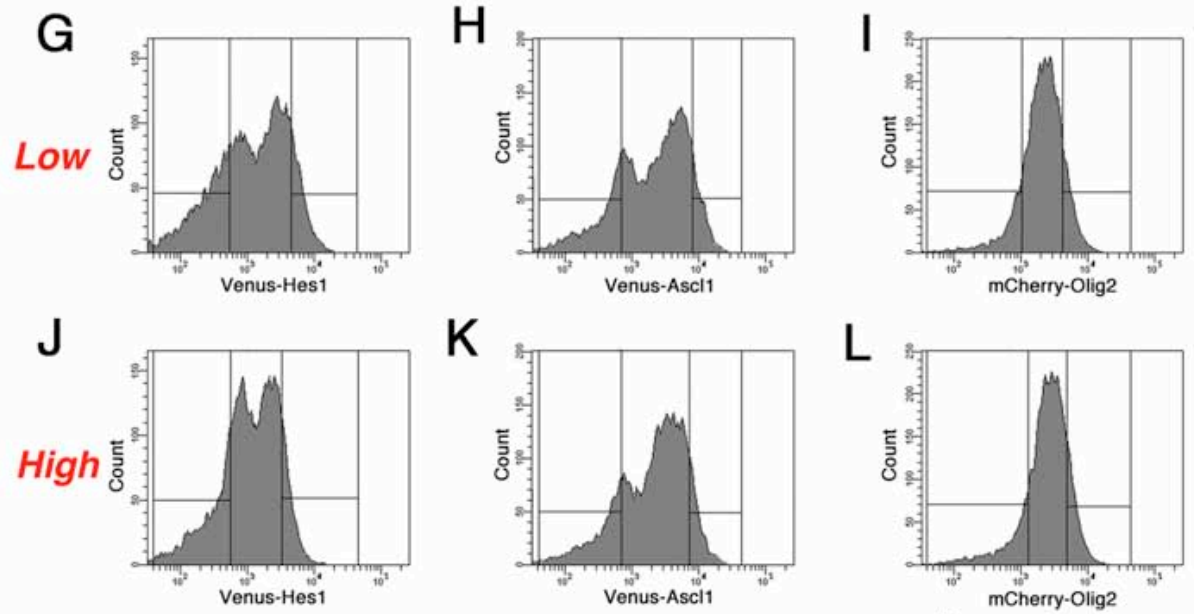

$\mathrm{K}$

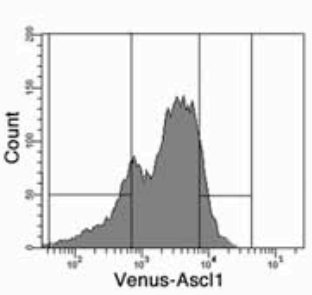

L
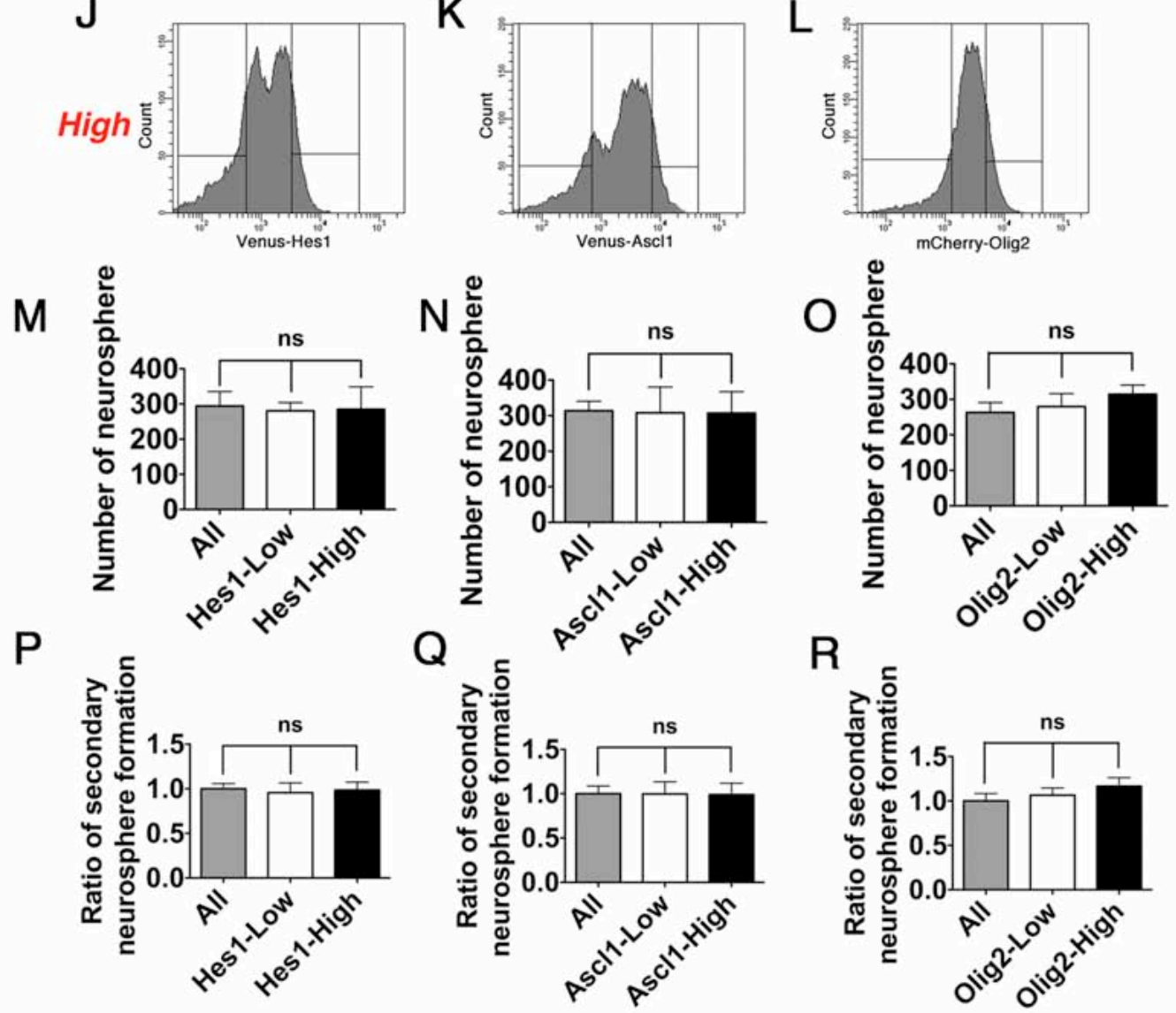
Fig. S16. FACS analysis of variable expression levels of bHLH factors in selfrenewing NPCs.

NPC cultures were prepared from fluorescent reporter-fusion knock-in mice or BAC Tg mice; Venus-Hes1 (A,D,G,J,M,P), Venus-Ascl1 (B,E,H,K,N,Q), or mCherry-Olig2 $(\mathbf{C , F}, \mathbf{I}, \mathbf{L}, \mathbf{O}, \mathbf{R})$. (A-C) Expression levels and dynamics of bHLH factors were analyzed by FACS. Hes1, Ascl1, and Olig2 expression levels were variable. (D-L) Venus- or mCherry-low cells (the lowest $10 \%$ of the whole population) and Venus- or mCherrryhigh cells (the highest 10\% of the whole population) were sorted and re-analyzed 2 days later. These populations returned to the original distribution of expression levels within 2 days when they were cultured in NS-cell culture medium. (M-R) Cells sorted by FACS were re-suspended in neurosphere medium, and plated in uncoated dishes. Primary neurospheres were counted 7 days after plating. Primary neurospheres were passaged and subjected to the secondary neurosphere assay. Hes1-high, Hes1-low, Ascl1-high, Ascl1low, Olig2-high and Olig2-low fractions generated neurospheres at similar efficiencies. $* p<0.05, * * p<0.01, * * * p<0.001, * * * * p<0.0001$, one-way ANOVA followed by Bonferroni post hoc test. 

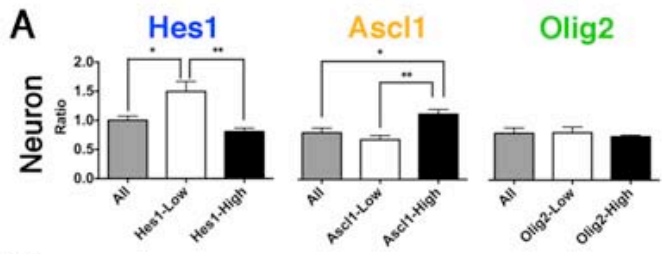

B
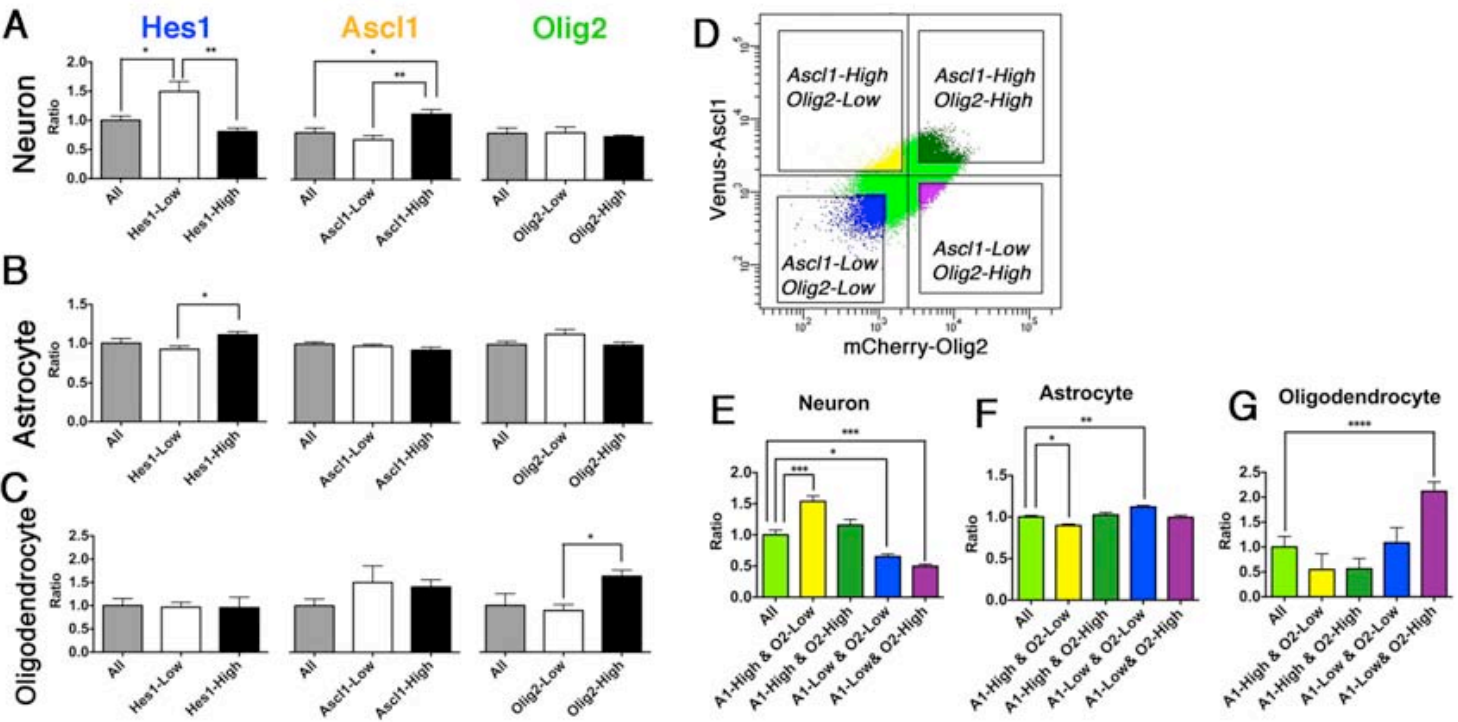

Fig. S17. Different expression levels of bHLH factors bias the fate choice of NPCs. NPC cultures were prepared from fluorescent reporter-fusion knock-in mice or BAC Tg mice. (A-C) NPCs were separated by FACS into Hes1-high, Hes1-low, Ascl1-high, Ascl1-low, Olig2-high and Olig2-low fractions. After sorting, each cell population was immediately transferred to a differentiation medium, and analyzed 7 days later by immunocytochemistry. (D-G) NPC cultures derived from Venus-Ascl1 and mCherryOlig2 double Tg mice were subjected to FACS analysis. 5\% of the total population depending on Ascl1 and Olig2 expression levels (Ascl1-high \& Olig2-low, Ascl1-high \& Olig2-high, Ascl1-low \& Olig2-low, or Ascl1-low \& Olig2-high) was sorted, and their differentiation competency was analyzed. $* p<0.05, * * p<0.01, * * * p<0.001, * * * * p<$ 0.0001 , one-way ANOVA followed by Bonferroni post hoc test. 
A

A

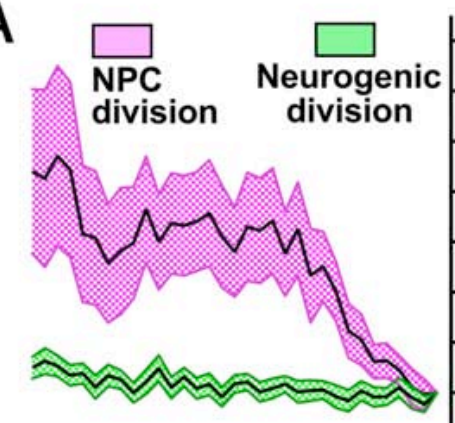

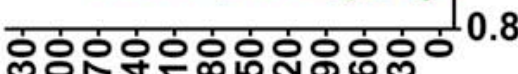

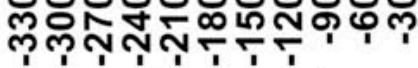

Time (min)
B Transient up-regulation before cell division

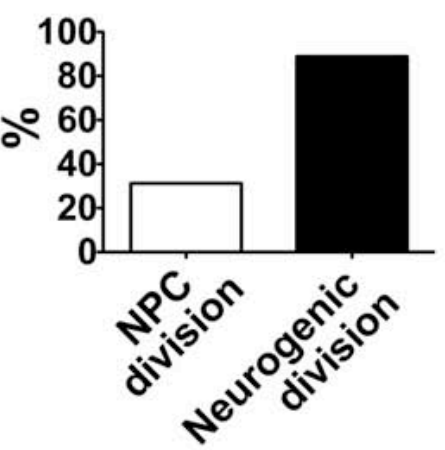

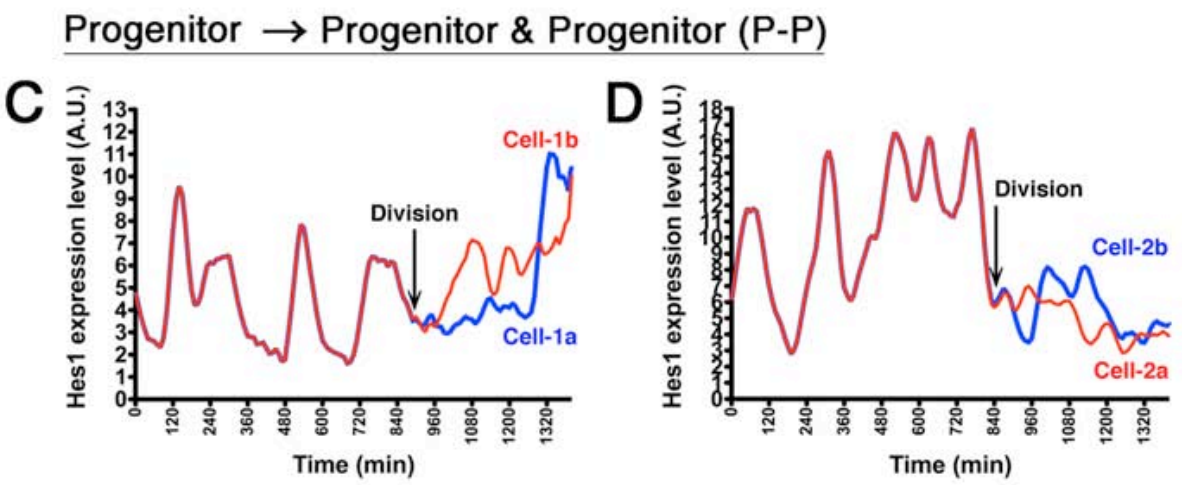

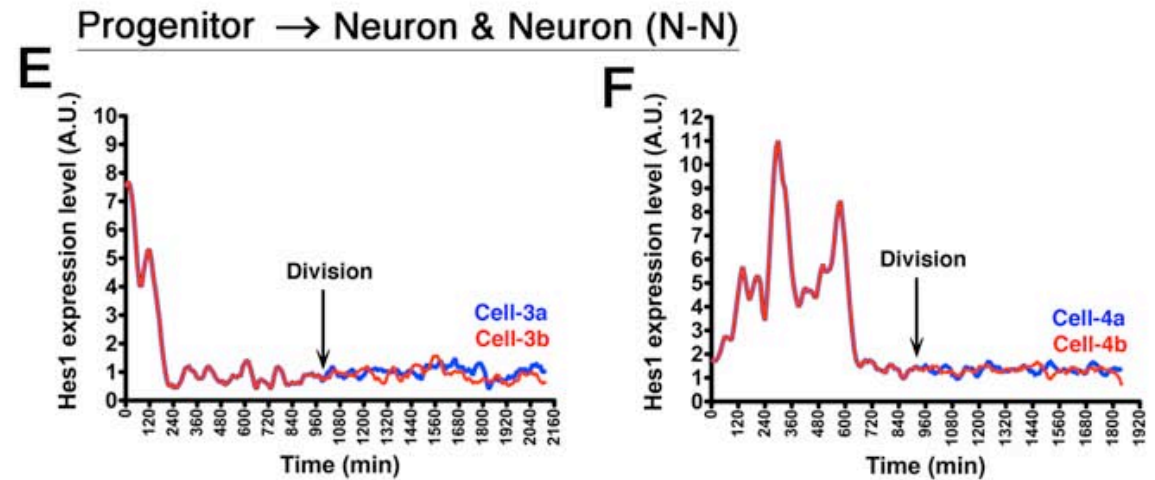

Fig. S18. Hes1 and Ascl1 expression dynamics during NPC division.

(A) Temporal trajectories of Luc2-Hes1 just before NPC or neurogenic division at time $=$ 0 (mean in solid line and standard errors in colored, $n>30$ for each division) were obtained by time-lapse analysis of NS cells derived from Luc2-Hes1 fusion BAC Tg mice (division-mode effect, $p=0.0016$; interaction between division-mode and time, $p<$ 0.0001 , repeated measures ANOVA). (B) The frequency of transient up-regulation (at least two-fold compared to the average) of Ascl1 expression before cell division. (C-F) Acute dissociation cultures were prepared from the ventral telencephalon of Luc2-Hes 1 fusion BAC Tg mice. Quantification of Luc2-Hes1 fusion protein dynamics during P-P $(\mathbf{C}, \mathbf{D})$ and N-N divisions $(\mathbf{E}, \mathbf{F})$. P-P, symmetric division that generates two NPCs; N-N, symmetric division that generates two neurons. 

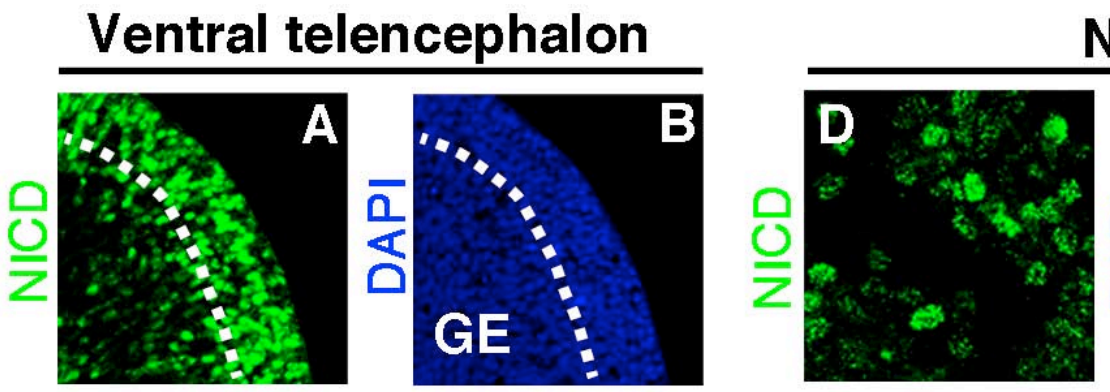

NPCs
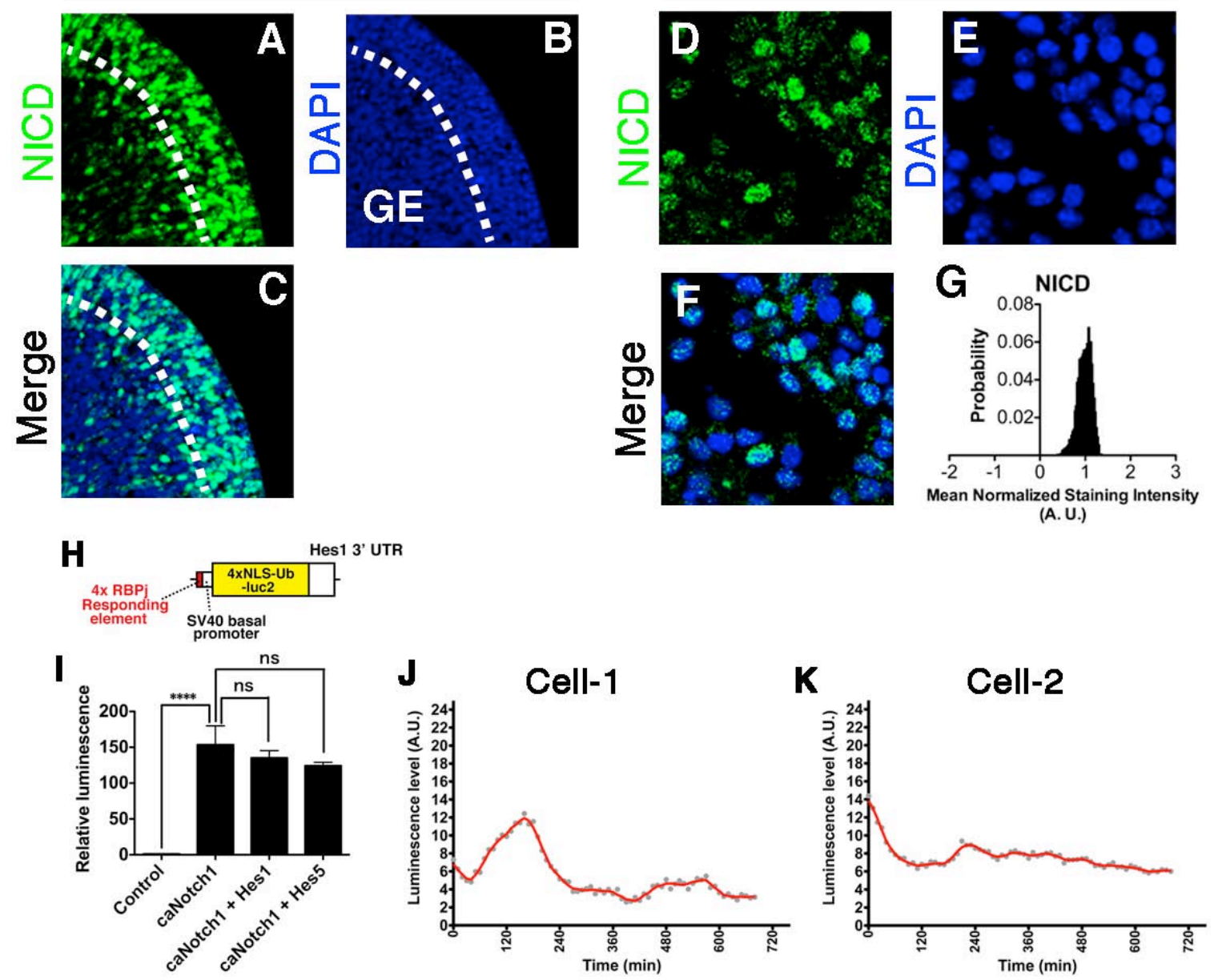

Fig. S19. Variable Notch signaling activities in NPCs.

(A-G) NICD immunofluorescence was analyzed in embryonic brain sections (A-C) and cultured NPCs (D-G). NICD levels were lower near the apical side, where cells at the late G2 and early G1 phases were present. The boundary between VZ and SVZ was

highlighted by white broken line. NICD expression levels were variable as that of bHLH factors in self-renewing NPCs (D-G). (H) Reporter construct for monitoring active NICD-RBPjk complex. (I) This reporter construct is not susceptible to Hes $1 / 5$ negative autoregulation because it does not contain Hes-binding sites, unlike the Hes promoters. Thus, the cellular activity of NICD-RBPjk complex can be faithfully monitored by this reporter. (J,K) The activity of NICD-RBPjk complex was dynamic in some NPCs $(\mathbf{J})$, but was less dynamic in others $(\mathbf{K}) . * * * * p<0.0001$, one-way ANOVA followed by Bonferroni post hoc test. 


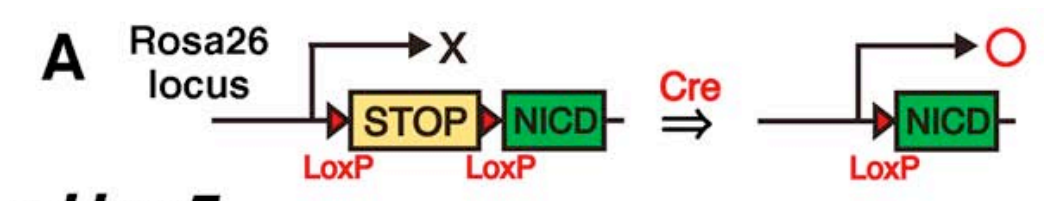

\section{pHes5}
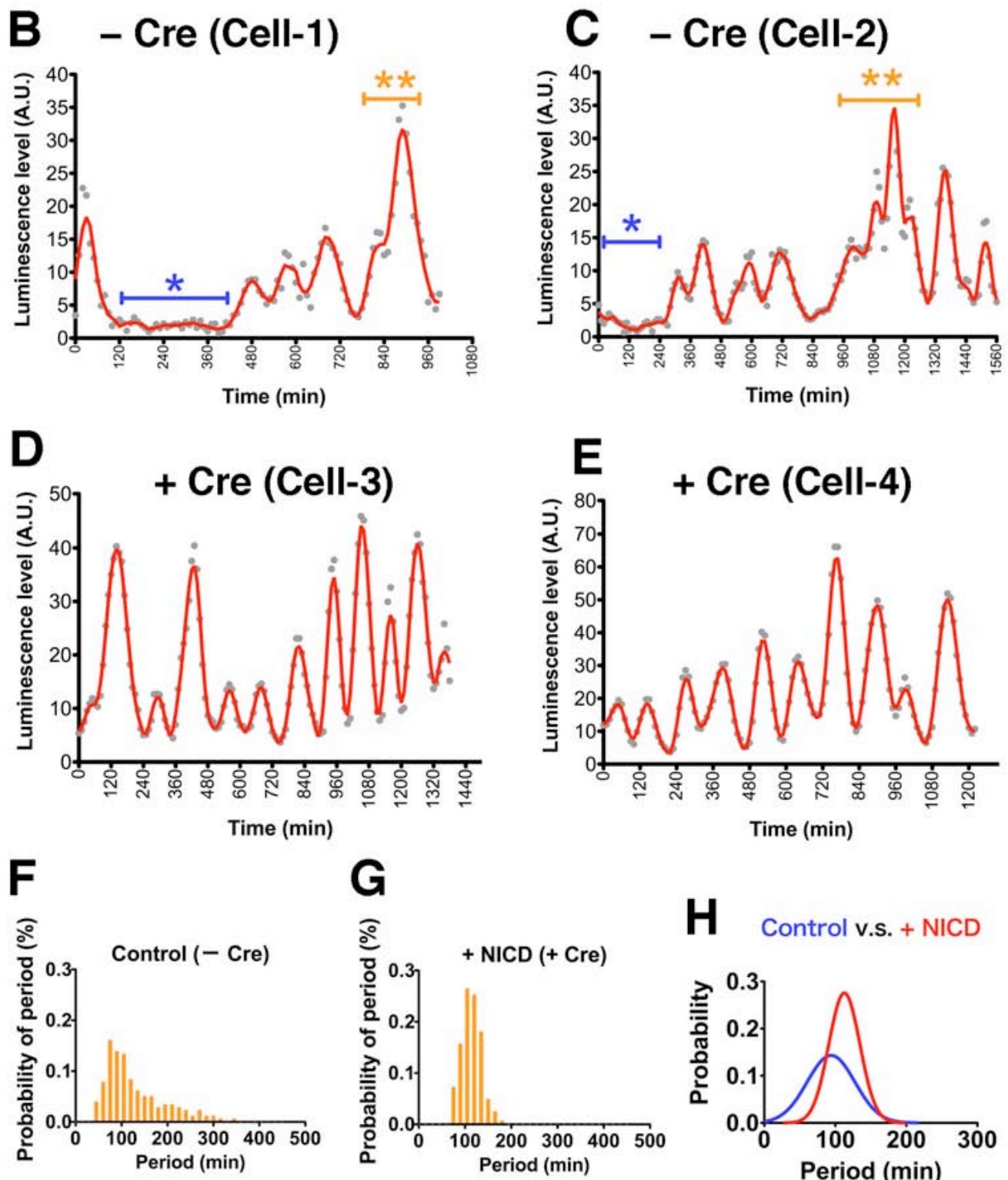

H Control v.s. + NICD

Fig. S20. Stable oscillation of Hes5 expression in NPCs whose Notch signaling is constantly active.

In NPCs, where NICD expression was constantly induced from the Rosa26 locus (A), oscillatory expression of Hes 5 was dramatically stable (D,E,G, + +Cre), compared with that in normal NPCs (B,C,F, -Cre). In normal NPCs, Hes 5 oscillation is frequently disrupted (B,C, blue and orange asterisks). Variation of Hes 5 oscillation periods in NPCs expressing NICD constantly was reduced compared with control $(\mathbf{F}-\mathbf{H})$. 


\section{A}

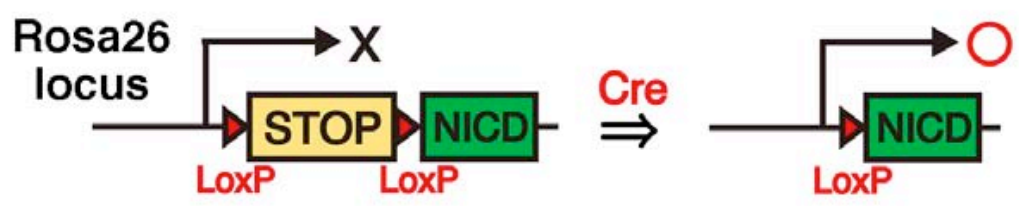

Hes1 protein
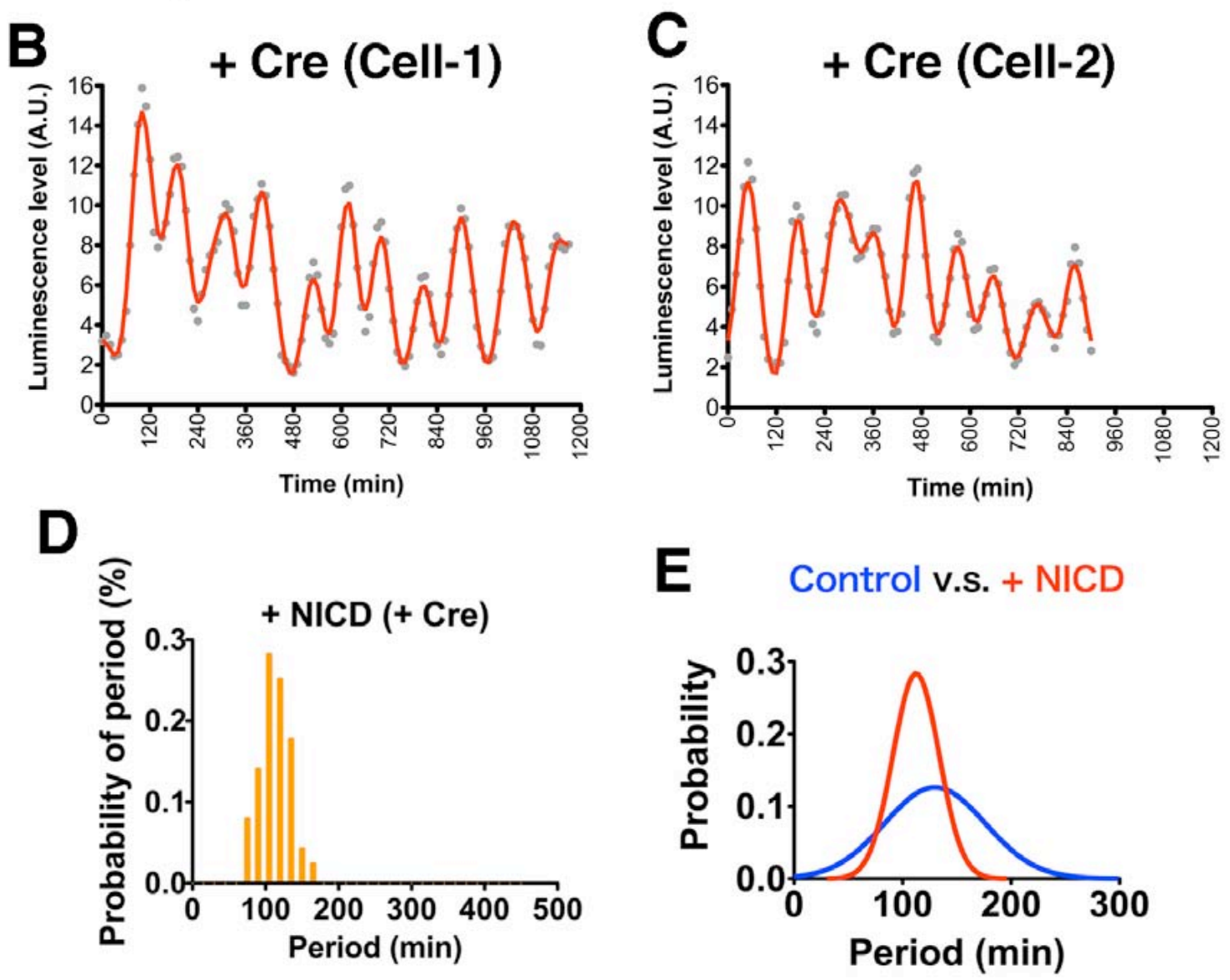

Fig. S21. Stable oscillation of Hes1 expression in NPCs whose Notch signaling is constantly active.

In NPCs, where NICD expression was constantly induced from the Rosa26 locus (A), oscillatory expression of Hes1 was dramatically stable $(\mathbf{B}, \mathbf{C}, \mathbf{D},+$ Cre $)$, compared with that in normal NPCs (Fig. 2A,G,H). Variation of Hes 1 oscillation periods in NPCs expressing NICD constantly was reduced compared with control (D,E). 
A Luc2-Ascl1 (Cell-population)

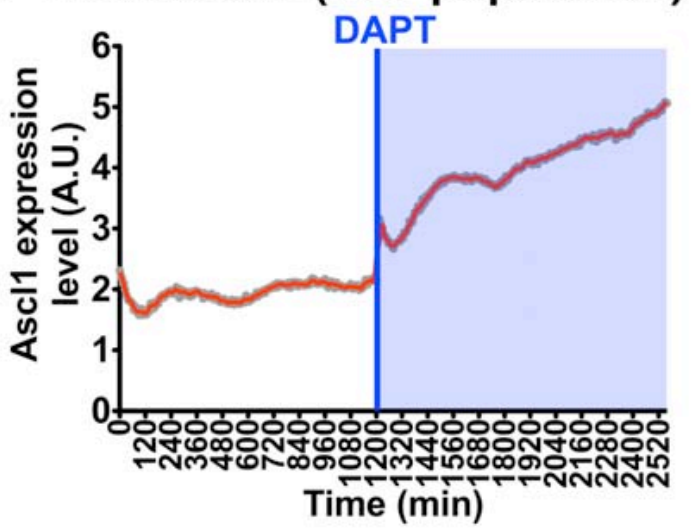

B Luc2-Ascl1 (Single cell)

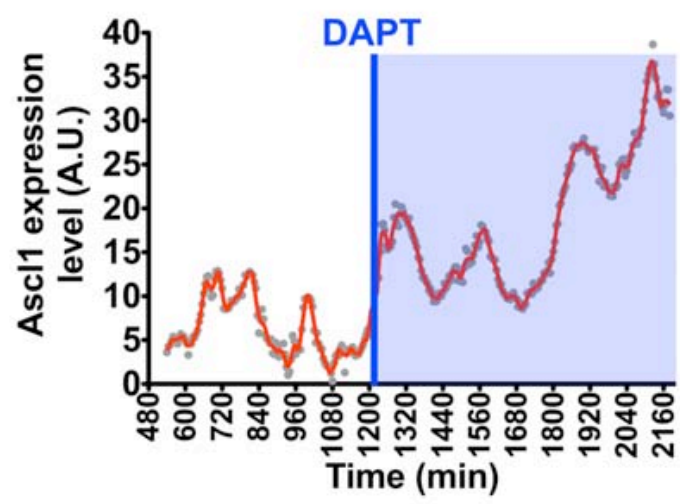

C Luc2-Olig2 (Cell-population)

D Luc2-Olig2 (Single cell)
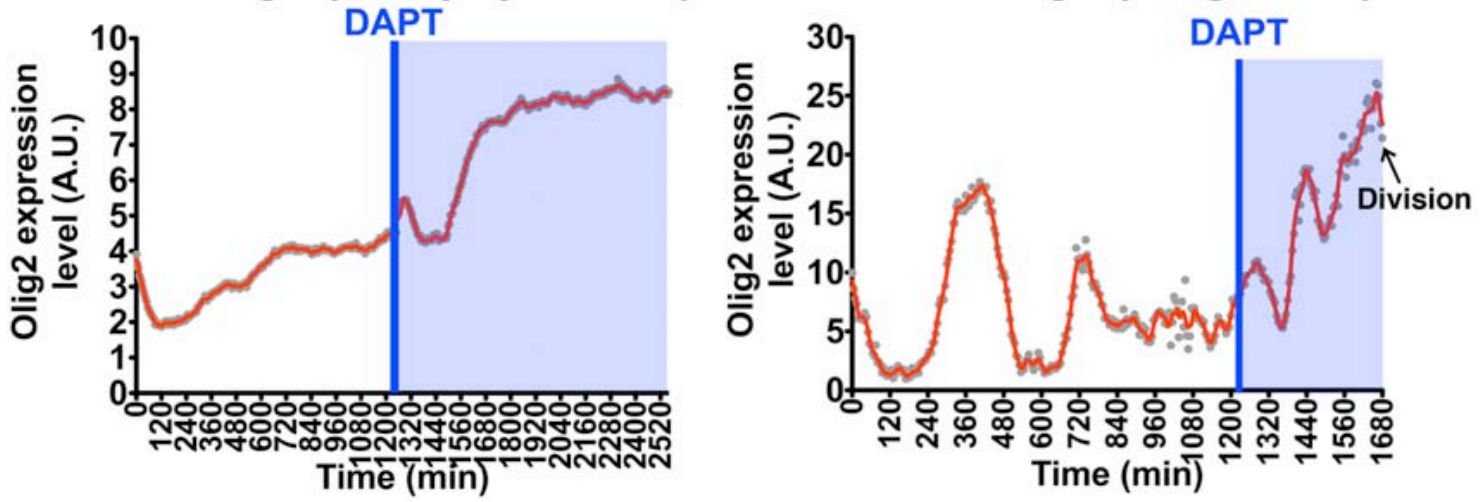

Fig. S22. Regulation of Ascl1 and Olig2 expression without Notch signaling. NPCs derived from Luc2-Asc11 (A,B) and Luc2-Olig2 (C,D) were treated with DAPT $(\gamma$-secretase inhibitor). Ascl1 and Olig2 expression was up-regulated in a sustained manner after DAPT treatment. Quantification of the reporter expression at cell population $(\mathbf{A}, \mathbf{C})$ and single cell levels $(\mathbf{B}, \mathbf{D})$ was displayed. 
A $\operatorname{LIF}(80 \mathrm{ng} / \mathrm{ml})$ BMP4 (80 $\mathrm{ng} / \mathrm{ml})$
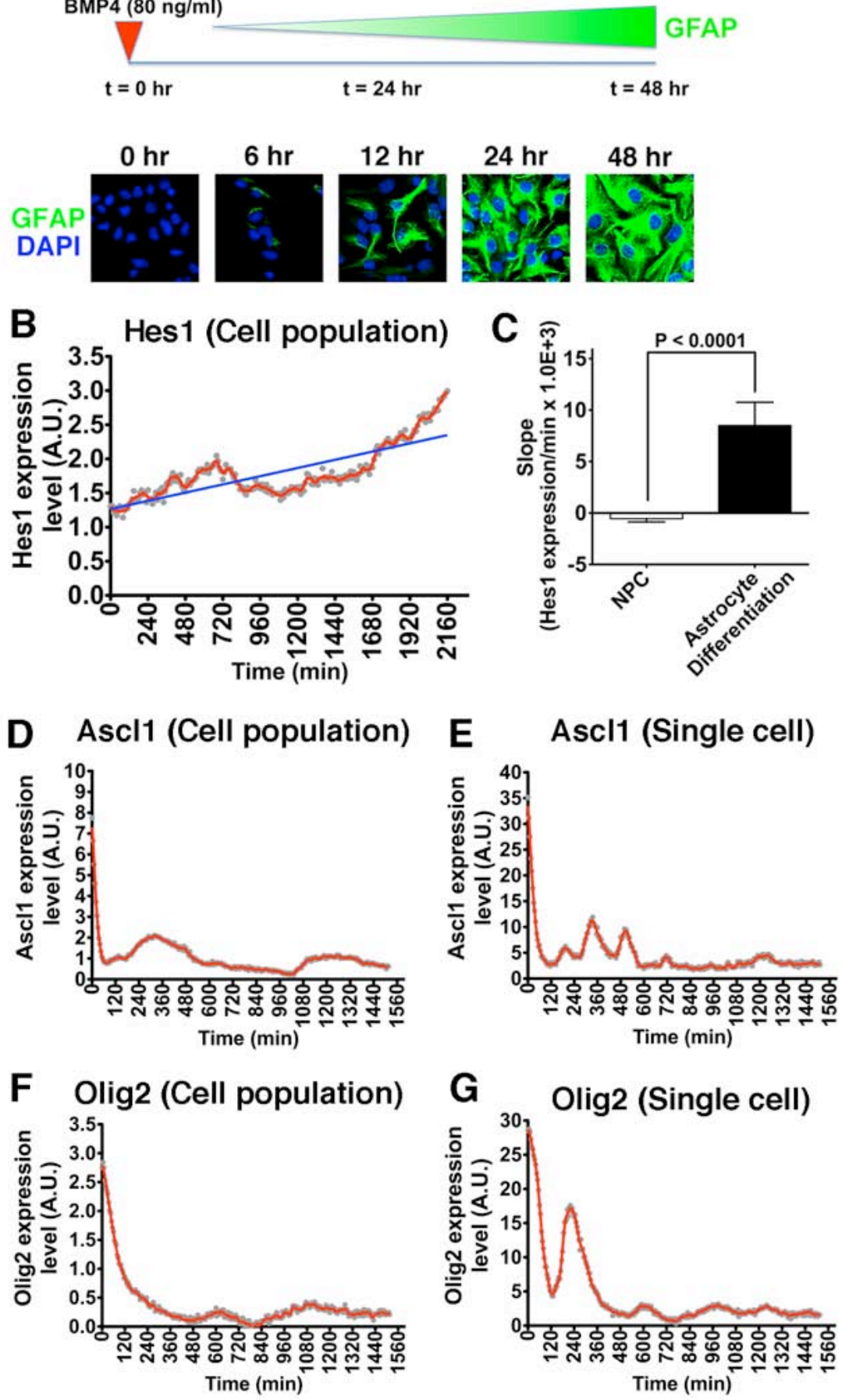

Fig. S23. Expression dynamics of bHLH factors during astrocyte differentiation.

(A) Astrocyte specification was induced at time $=0$ by LIF and BMP4. GFAP expression was induced. (B,C) Quantification of Hes1 up-regulation during astrocyte differentiation. NPC, $n=30 ;$ Astrocyte differentiation, $n=21$. Significance was analyzed by two-tailed Student's $t$-test. (D-G) Quantification of Luc2-Asc11 (D,E) and Luc2-Olig2 (F,G) expression during astrocyte differentiation. Quantification of the reporter expression at cell population $(\mathbf{B}, \mathbf{D}, \mathbf{F})$ and single cell levels $(\mathbf{E}, \mathbf{G})$ was displayed. 

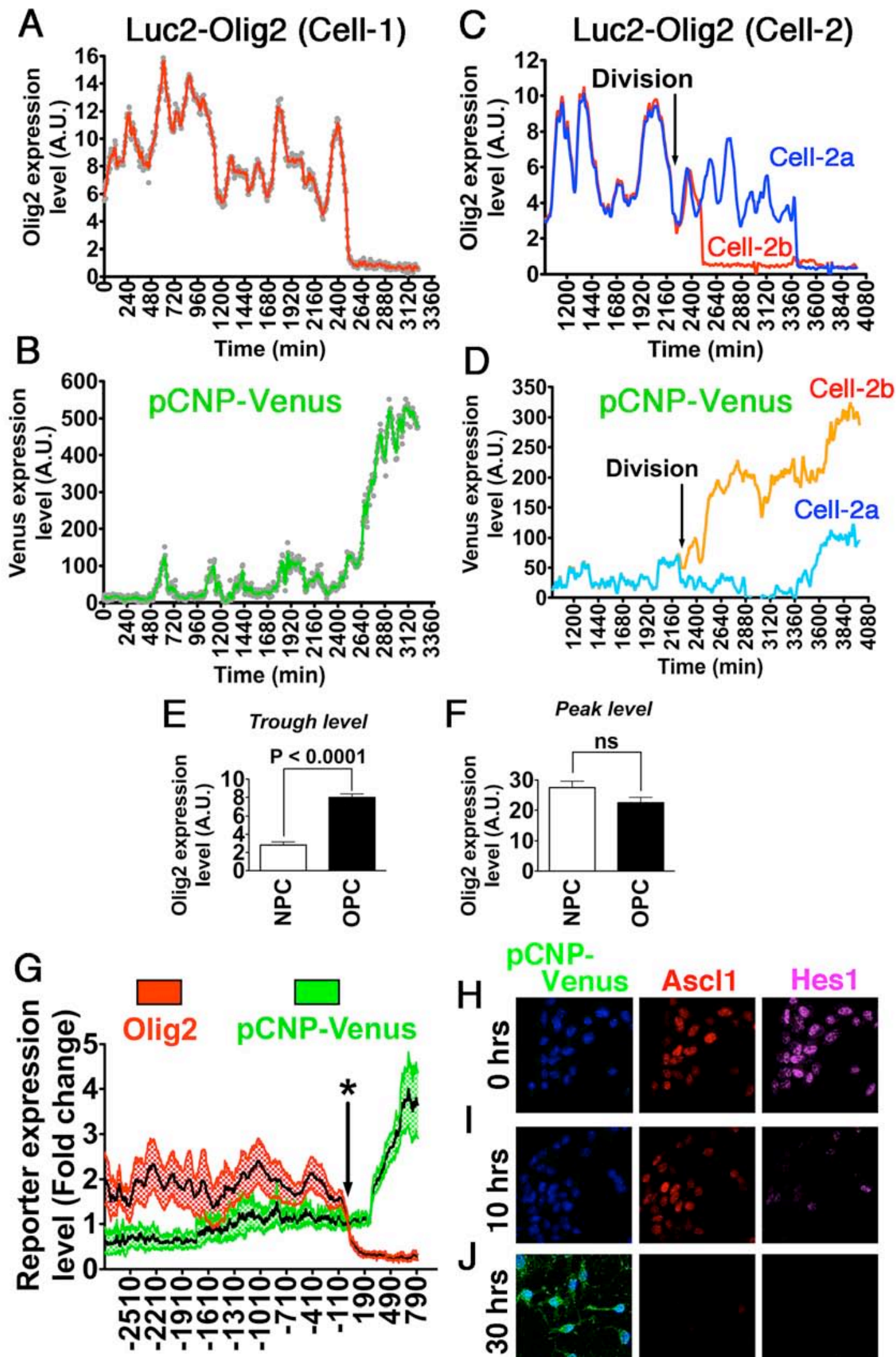

Time (min)

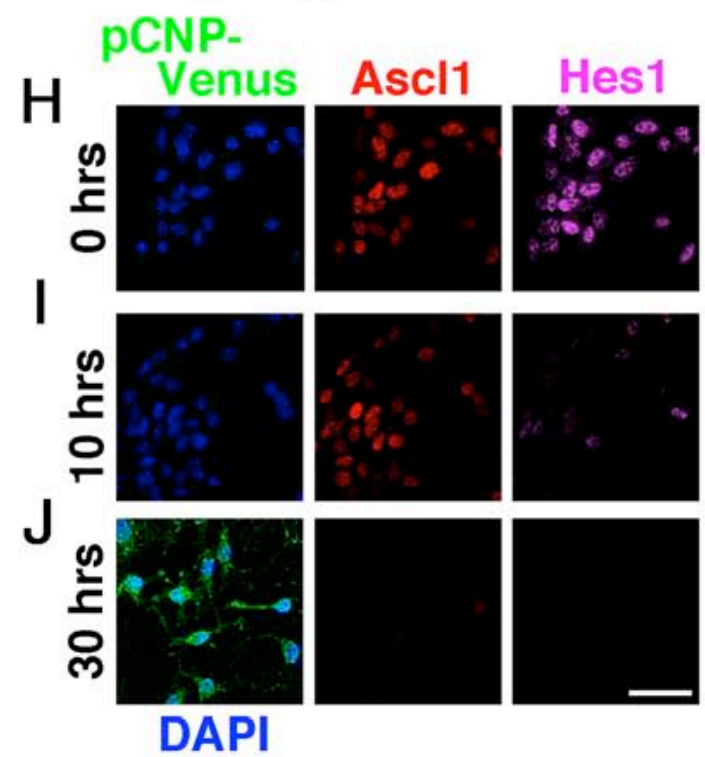




\section{Fig. S24. Expression dynamics of bHLH factors during oligodendrocyte} differentiation.

$(\mathbf{A}, \mathbf{B})$ Quantification of Luc2-Olig2 (A) and pCNP-Venus expression (B) in a single cell during oligodendrocyte differentiation, which was induced at time $=0$ by T3. (C,D) Quantification of Luc2-Olig2 (C) and pCNP-Venus (D) expression in the same cells (Cell-2 and their daughter cells, Cell-2a and cell-2b) during oligodendrocyte differentiation, which was induced at time $=0$ by T3. (E,F) Comparison of Olig2 expression levels at trough $(\mathbf{E})$ and peak $(\mathbf{F})$ of oscillation between NPCs and OPCs (oligodendrocyte precursor cells). Olig2 expression level in OPCs was significantly higher at trough than that in NPCs. Significance was analyzed by two-tailed Student's $t$ test. (G) Temporal trajectories ( $\mathrm{n}>16$ cells) of Luc2-Olig2 (mean in solid line and standard errors in colored) and pCNP-Venus (mean in solid line and standard errors in colored). Time $=0$ (asterisk) corresponds to time of rapid Olig2 down-regulation. $(\mathbf{H}-\mathbf{J})$ Ascl1 and Hes1 expression during oligodendrocyte differentiation. Scale bar, $12.5 \mu \mathrm{m}(\mathbf{H}-$ J). 
A pCNP-Venus $\mathrm{Tg}$ mouse
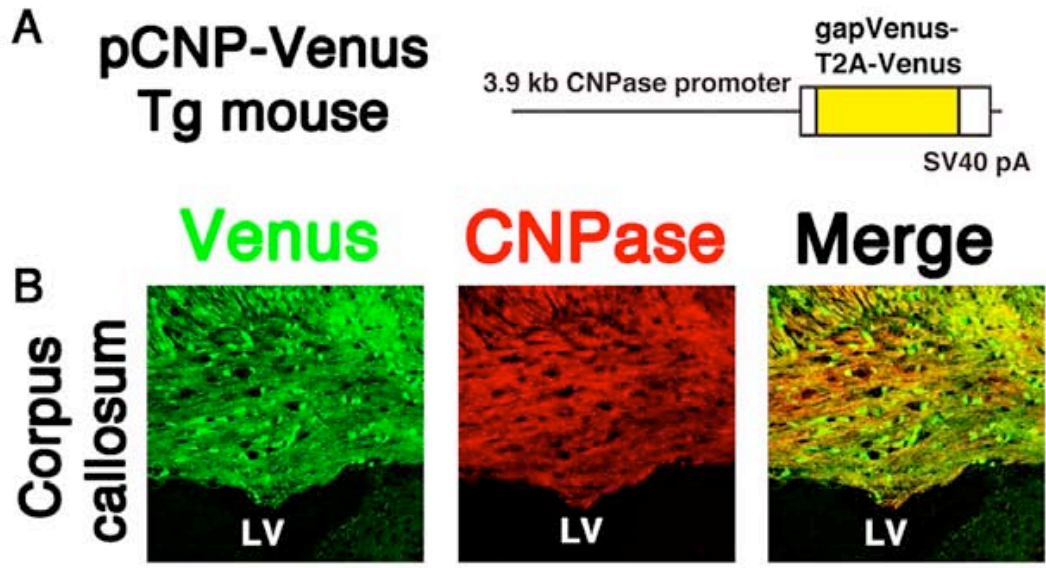

CNPase Merge
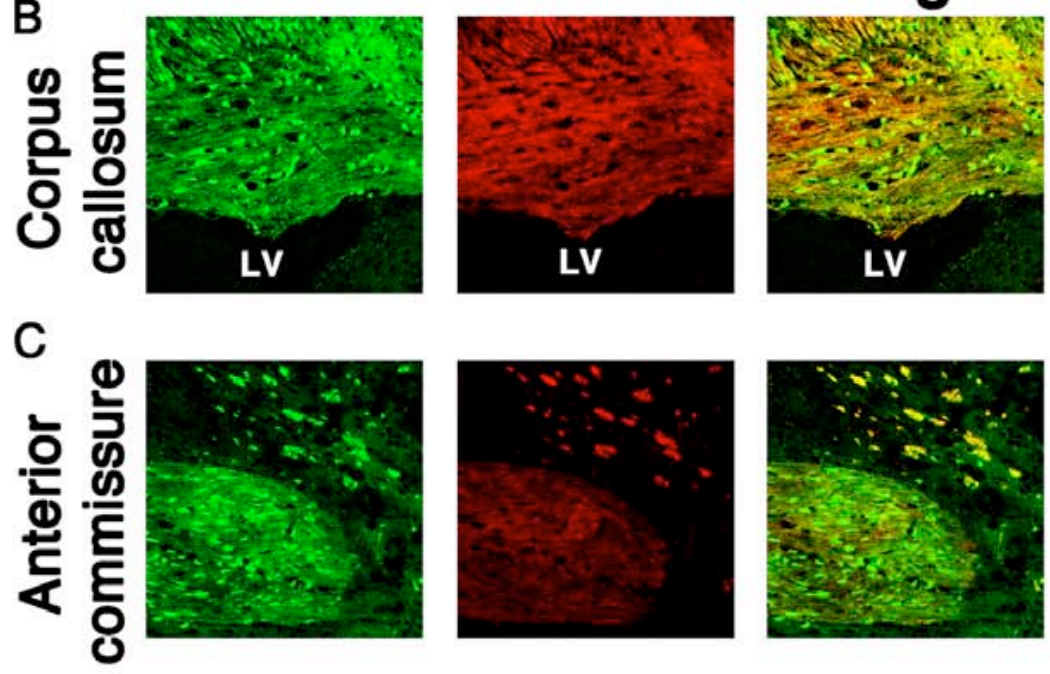

D
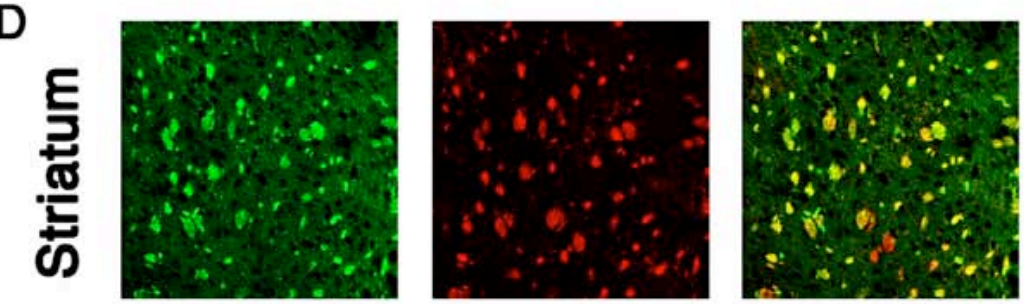

$\mathrm{E}$
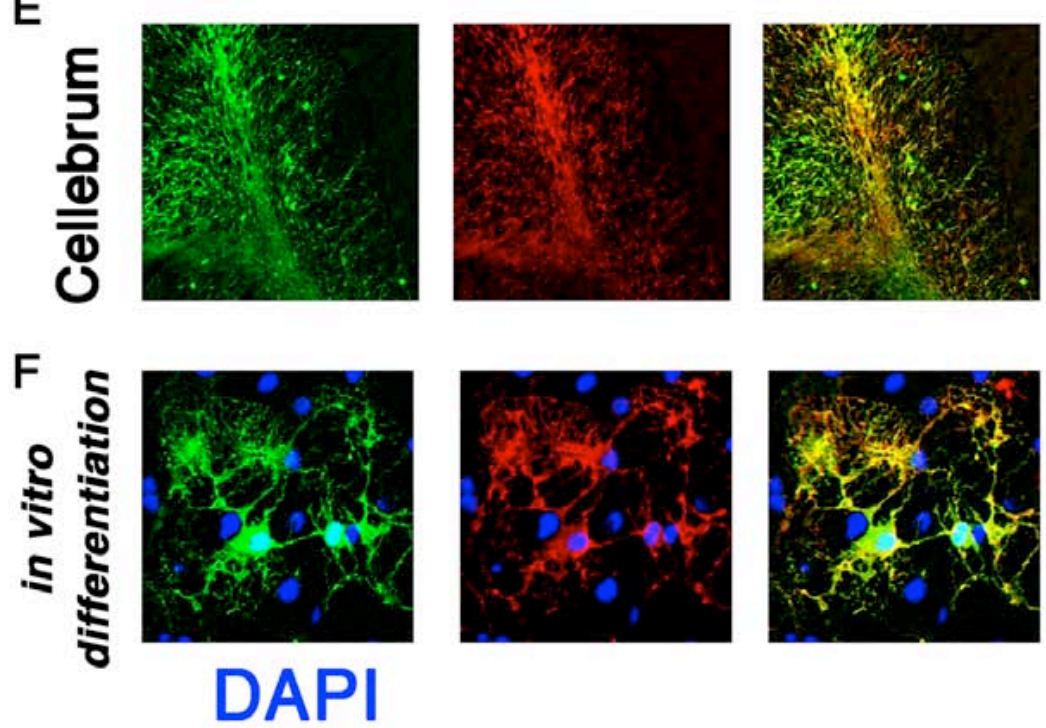

Fig. S25. Characterization of pCNP-Venus Tg mice.

(A) Schematic illustration of pCNP-Venus Tg construct. (B-F) Venus expression (green) was analyzed and compared with endogeneous CNPase expression (red) in the adult brain (B-E) and differentiated oligodendrocytes in vitro $(\mathbf{F})$. 

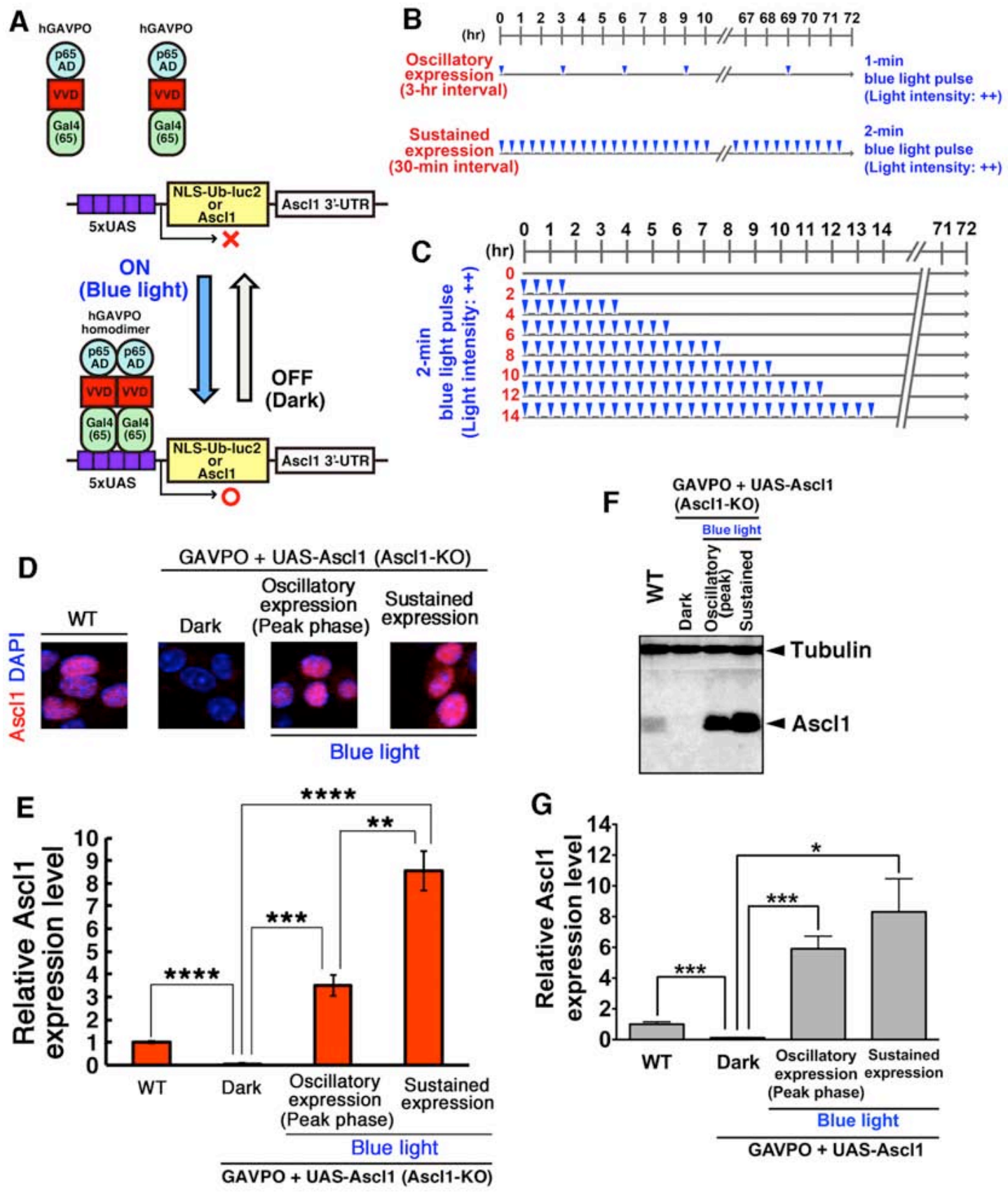

Fig. S26. Experimental design for light-induced oscillatory/sustained expression of Ascl1 in NPCs.

(A) Strategy for the light-induced gene expression using humanized GAVPO (hGAVPO). (B) The schedule of light exposure. Ascl1 expression was induced in Ascl1-null NPCs. Repeated exposure of blue light with 3-hr intervals generated oscillatory expression with a 3-hr period, while repeated exposure with 30-min intervals generated sustained expression (Fig. 5A-K). (C) The schedule of light exposure. Sustained Ascl1 expression was induced in NPCs for indicated time lengths (Fig. 5L-P). Light intensity: ++, $1.11 \mu \mathrm{mol} / \mathrm{m}^{2} / \mathrm{s}$. (D-G) Ascl1 expression was induced in Ascll-null NPCs according to the schedule of light exposure (fig. S26B) and examined at $\mathrm{t}=250 \mathrm{~min}$ and $360 \mathrm{~min}$ in oscillatory and sustained expression, respectively (the light-induced condition was the 
same as Fig. 5A-C). Representative images (D) and their quantitative data (E) of immunocytochemistry and western blot $(\mathbf{F})$ and its quantitative data $(\mathbf{G})$ are shown. Quantitative data (mean \pm SEM) are expressed as fold-change over wild-type control. For western, Ascl1 protein levels were normalized using anti-tubulin. Here, the peak level of the light-induced Ascll oscillation and the level of the light-induced sustained Ascl1 expression were 3 to 6 -fold and $\sim 8$-fold higher, respectively, than the endogenous average level in WT NPCs (E,G). Peaks of endogenous Ascl1 expression are several-fold higher than the average level of NPCs (Fig. 3, fig. S11), and therefore these light-induced levels are comparable to the endogenous peak levels in NPCs and differentiating neurons. ${ }^{*} p<0.05, * * p<0.01,{ }^{* * *} p<0.001, * * * * p<0.0001$; two-tailed Student's $t$-test. 
A

(hr)

$\begin{array}{lllllllllllll}0 & 1 & 2 & 3 & 4 & 5 & 6 & 7 & 8 & 9 & 10 & 1112\end{array}$

69707172

Oscillatory

(6-hr interval)

1-min

blue light pulse

\section{Light-induced oscillatory expression (Ascl1-KO NPC) 1-min light pulse with 6hr-period}
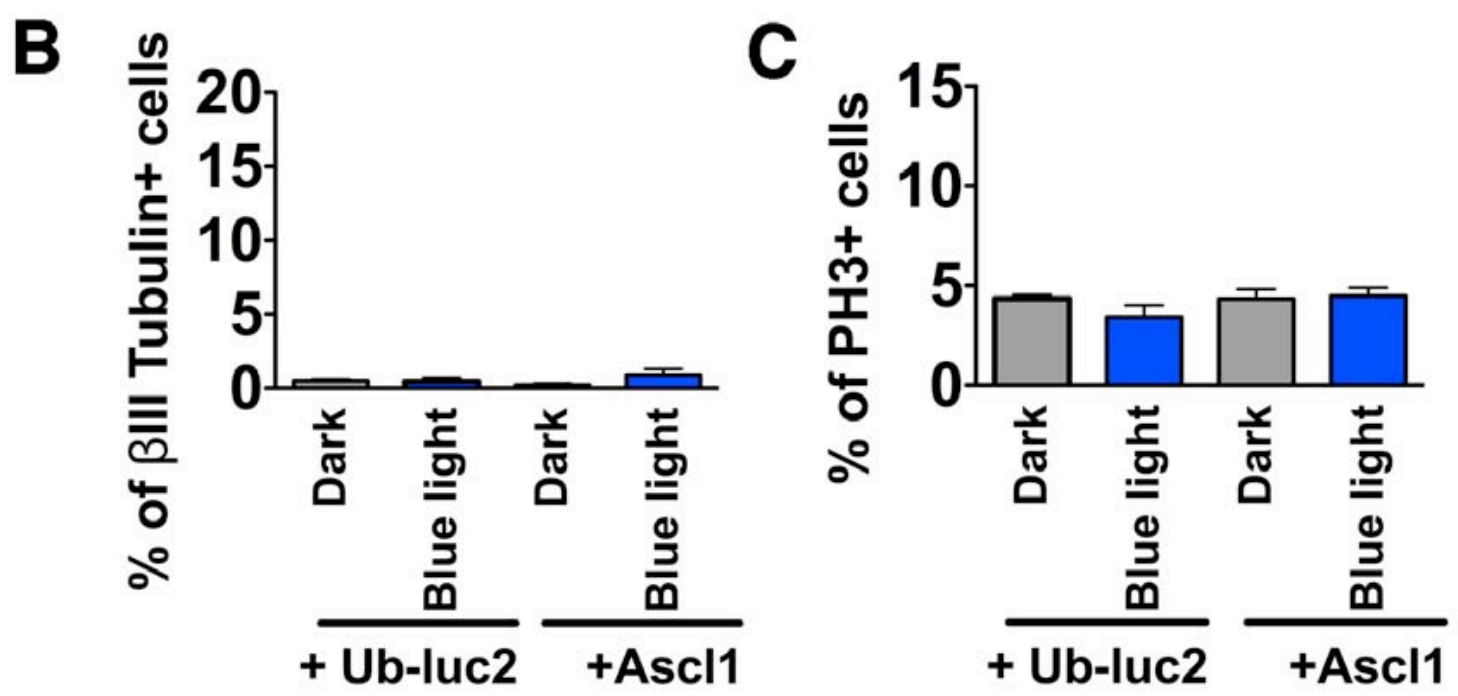

Fig. S27. Oscillatory Ascl1 expression with 6-hour period did not affect the proportion of dividing cells in Ascll-null NPCs.

(A) The schedule of light exposure. Oscillatory Ascl1 expression with 6-hr period was induced in Ascl1-null NPCs, which were cultured in the presence of bFGF and EGF. (B,C) Oscillatory Ascl1 expression with 6-hr period induced virtually no $\beta$ III tubulin ${ }^{+}$ neuron formation $(\mathbf{B})$ and did not significantly increase the proportion of dividing cells $\left(\mathrm{PH}^{+}\right)(\mathbf{C})$. Significance was analyzed by two-tailed Student's $t$-test. 

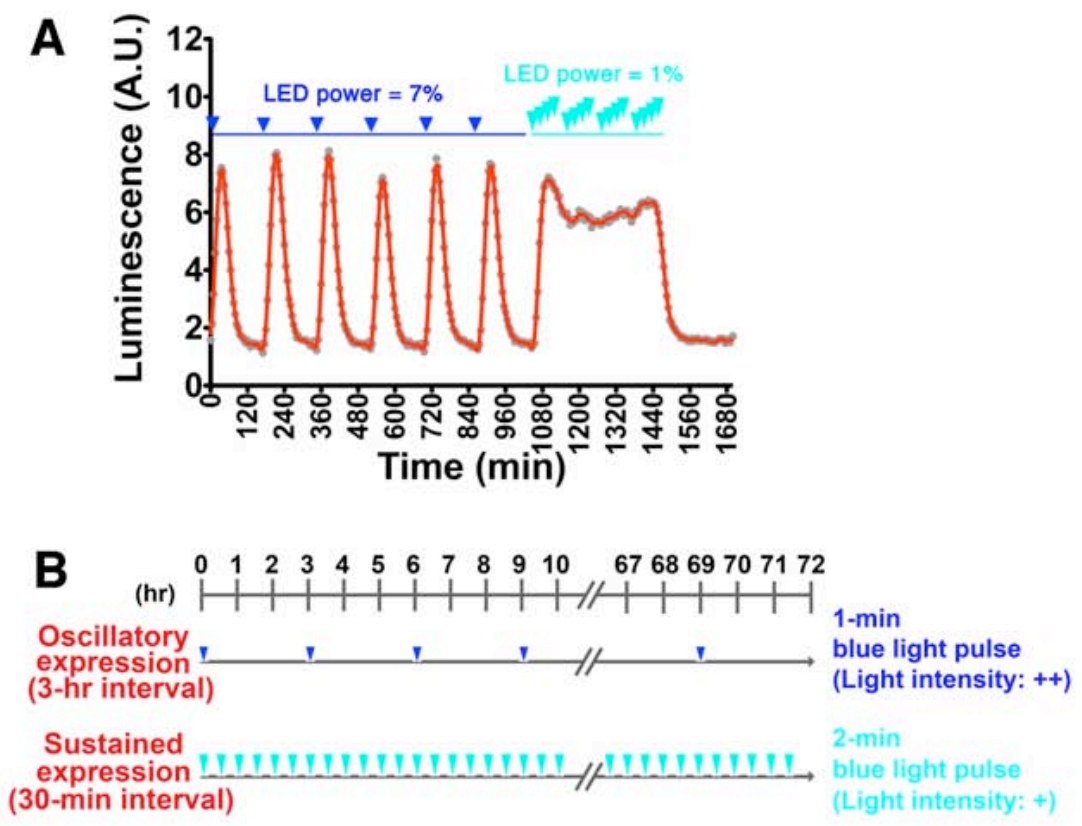

Light-induced oscillatory expression (Ascl1-KO NPC) 1-min light pulse (Light intensity: ++ )

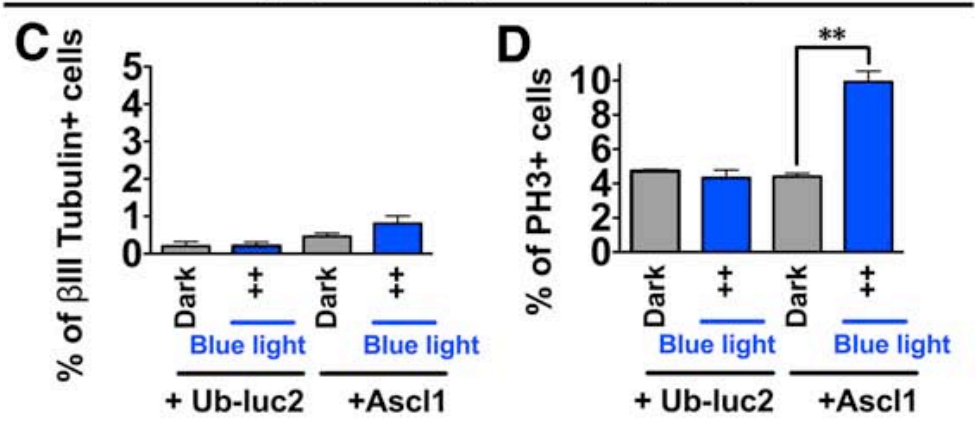

Light-induced sustained expression (Ascl1-KO NPC) 2-min light pulse (Light intensity: +)
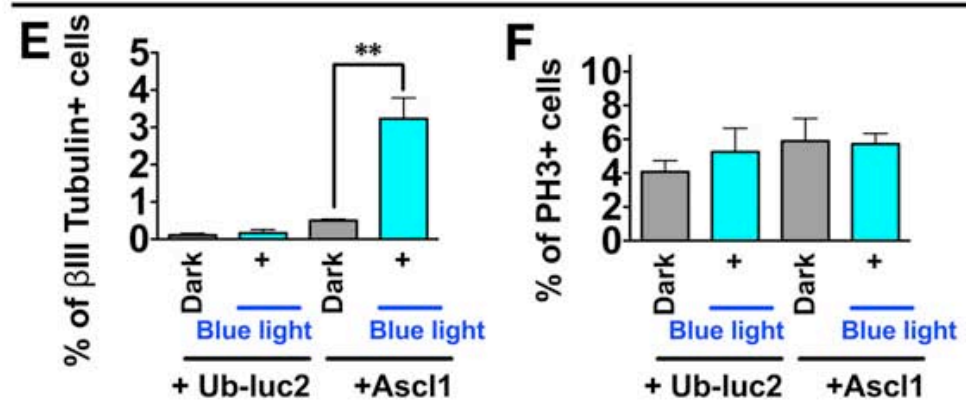

Fig. S28. Sustained Ascl1 expression at lower levels induces neuronal differentiation in Ascll-null NPCs.

(A) Repeated exposure with 30-min intervals by 1\% LED power achieved sustained expression at comparable expression levels with the peak levels of oscillatory expression generated by blue light exposure with 3-hr intervals by 7\% LED power. (B) The schedule of light exposure. Ascl1 expression was induced in Ascl1-null NPCs, which were 
cultured in the presence of $\mathrm{bFGF}$ and EGF, a condition that inhibits neurogenesis. Light intensity:,$+ 0.16 \mu \mathrm{mol} / \mathrm{m}^{2} / \mathrm{s} ;++, 1.11 \mu \mathrm{mol} / \mathrm{m}^{2} / \mathrm{s}$. (C-F) Oscillatory Ascl1 expression induced virtually no $\beta$ III tubulin ${ }^{+}$neuron formation $(\mathbf{C})$ but significantly increased the proportion of dividing cells $\left(\mathrm{PH}^{+}\right)$, compared to the control (Ub-luc2) (D). Sustained Ascl1 expression at lower levels significantly increased $\beta$ III tubulin ${ }^{+}$neuron formation (E) but did not affect the proportion of dividing cells $\left(\mathrm{PH}^{+}\right)$, compared to the control (Ub-luc2) (F). Data sets used in (C,D) were the same as that of Fig. 5D,E. *, $p<0.05$; **, $p<0.01$; two-tailed Student's $t$-test. 
A

(hr)

$\begin{array}{lllllllllll}0 & 1 & 2 & 3 & 4 & 5 & 6 & 7 & 8 & 9 & 10\end{array}$

676869707172

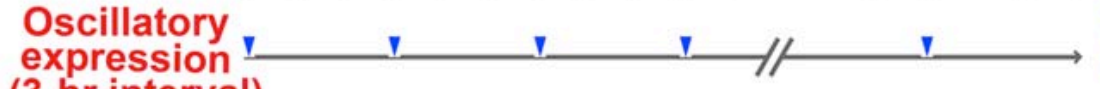

(3-hr interval)

1-min

blue light pulse

2-min

Sustained

blue light pulse (30-min interval)

Light-induced oscillatory Ascl1 expression (Wild-type NPC)
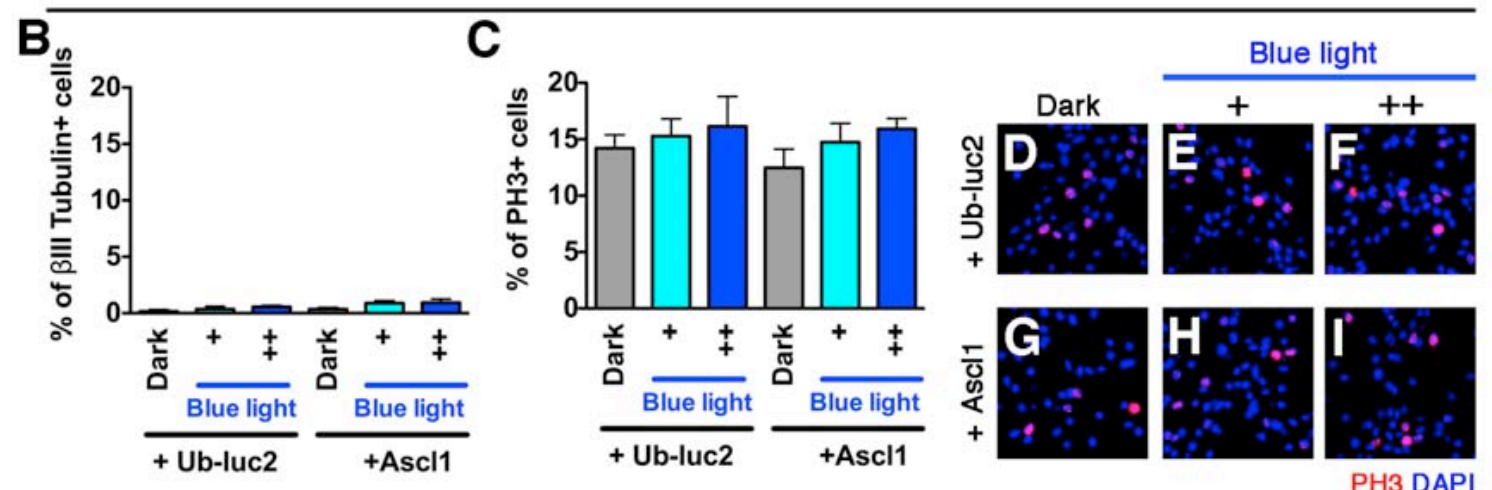

Light-induced sustained Ascl1 expression (Wild-type NPC)

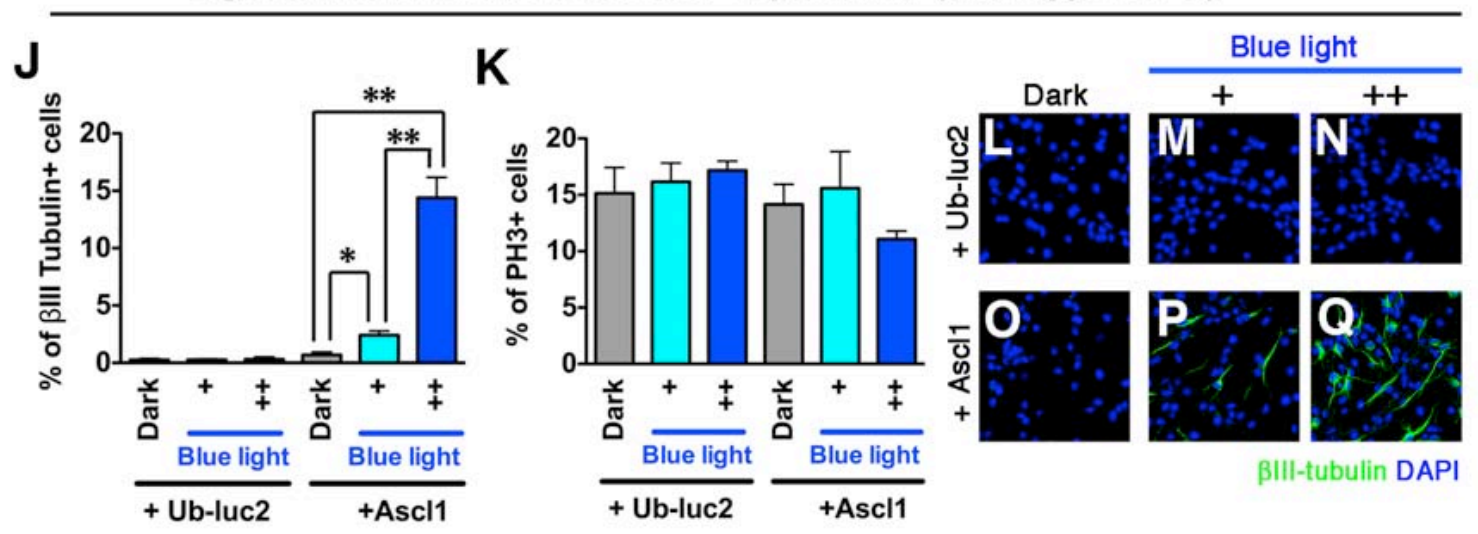

Fig. S29. Light-induced oscillatory/sustained expression of Ascl1 in wild-type NPCs. According to the schedule of light exposure (A), oscillatory (B-I) and sustained (J-Q) Ascll expression was induced in wild-type NPCs, which were cultured in the presence of bFGF and EGF, a condition that inhibits neurogenesis. Oscillatory light stimulation of a 3-hr period did not significantly affect the neurogenesis or proliferation of these cells (BI). By contrast, sustained Ascl1 expression for $72 \mathrm{hr}$ significantly increased neuronal formation with some tendency to reduce the proportion of dividing cells $(\mathbf{J}-\mathbf{Q})$. Light intensity:,$+ 0.16 \mu \mathrm{mol} / \mathrm{m}^{2} / \mathrm{s} ;++, 1.11 \mu \mathrm{mol} / \mathrm{m}^{2} / \mathrm{s}$. The proportion of dividing cells was measured by immunostaining with anti-PH3 antibody. Cells having punctated signals in the nuclei were included in the PH3-immunopositive fraction in this study. ${ }^{*} p<0.05,{ }^{* *} p$ $<0.01$; two-tailed Student's $t$-test. 

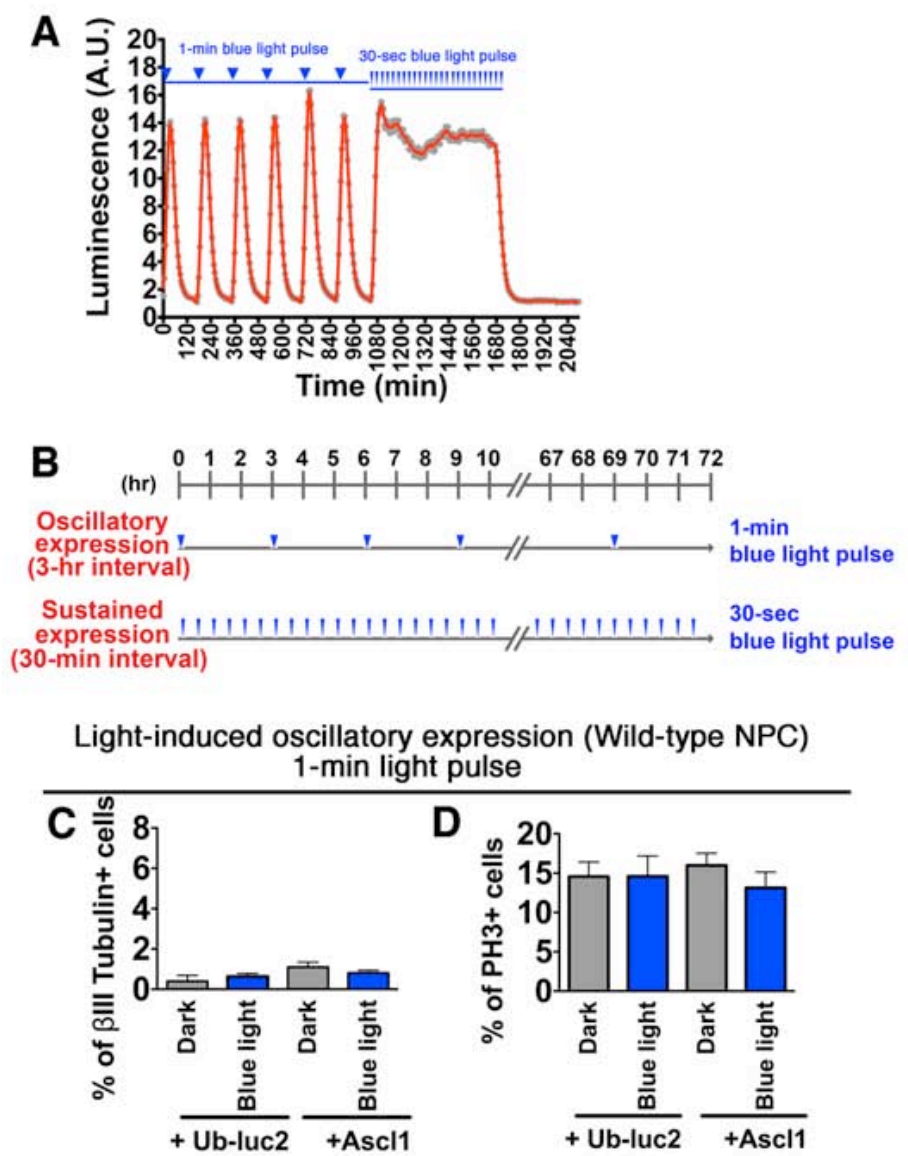

Light-induced sustained expression (Wild-type NPC) 30 -sec light pulse
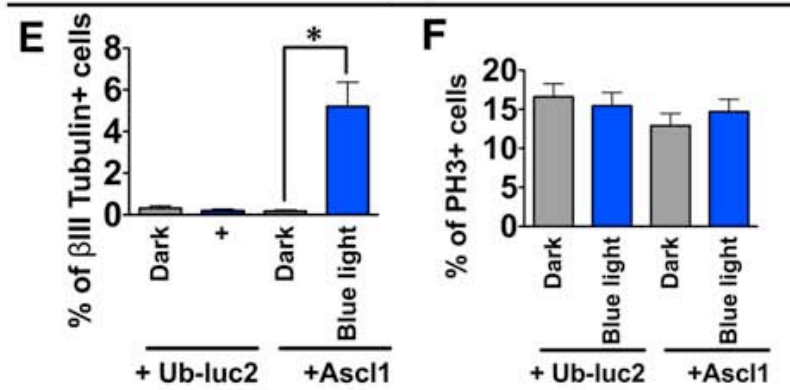

Fig. S30. Sustained Ascl1 expression at lower levels induces neuronal differentiation in wild-type NPCs.

(A) 30-sec blue-light pulse with 30-min intervals achieved sustained expression at comparable expression levels with the peak levels of oscillatory expression generated by 1-min blue-light pulse with 3-hr intervals. (B) The schedule of light exposure. Ascl1 expression was induced in wild-type NPCs, which were cultured in the presence of bFGF and EGF. Light intensity: $1.11 \mu \mathrm{mol} / \mathrm{m}^{2} / \mathrm{s}$. (C-F) Oscillatory light stimulation with 3-hr period did not significantly affect the neurogenesis or proliferation of these cells $(\mathbf{C , D})$. By contrast, sustained Ascl1 expression at low levels for $72 \mathrm{hr}$ significantly increased $\beta$ III tubulin ${ }^{+}$neuron formation $(\mathbf{E})$ but did not affect the proportion of dividing cells $\left(\mathrm{PH}^{+}\right)(\mathbf{F}) .{ }^{*} p<0.05$; two-tailed Student's $t$-test. 

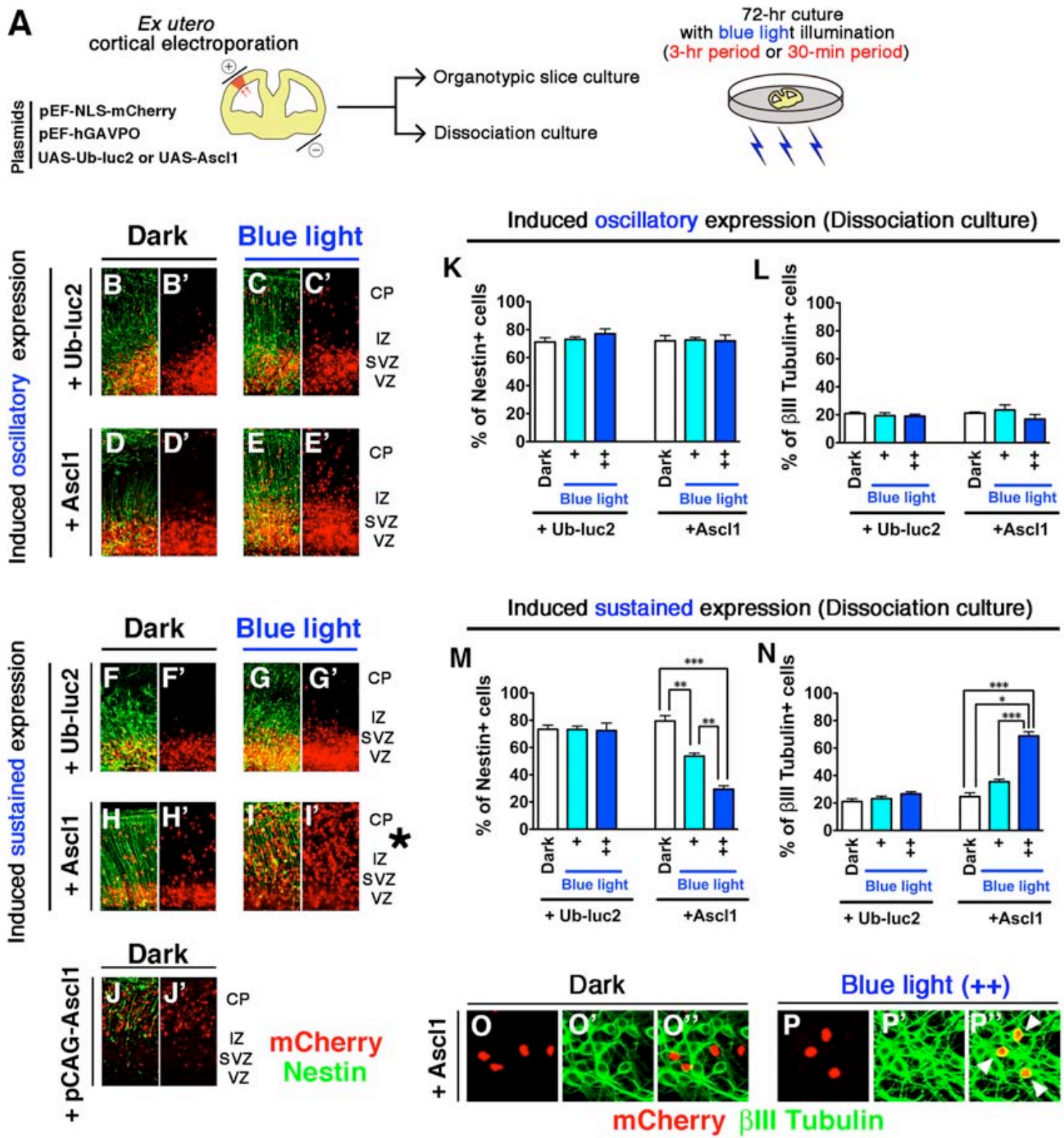

\section{Fig. S31. Light-induced oscillatory/sustained expression of Ascl1 in dorsal} telencephalic NPCs.

(A) The Ascl1-inducible system was introduced into the dorsal telencephalon, which normally expresses very low levels of Ascl1, by ex utero electroporation. Transfected brains were immediately sliced or dissociated, and cultured for $72 \mathrm{hr}$ under the periodic blue-light illumination, and then analyzed by immunostaining. (B-J') Representative images of cortical slices, indicating distribution of the transfection marker (red; NLSmCherry) and NPC marker (green, Nestin). The oscillatory expression of Ascl1 maintained Nestin ${ }^{+}$NPCs in the ventricular zone (E,E'), while the sustained expression of Ascl1 increased the number of cells that migrated into the IZ/CP (I,I', asterisk), resembling well the results in which Ascl1 was over-expressed by constitutively active CAG promoter $\left(\mathbf{J}, \mathbf{J}\right.$ '). Light intensity $=1.11 \mu \mathrm{mol} / \mathrm{m}^{2} / \mathrm{s}$. (K-P'') According to the schedule of light exposure, oscillatory (K,L) and sustained (M-P'') Ascl1 expression was induced in dissociated NPCs derived from electroporated brains. Oscillatory light 
stimulation with 3-hr period did not significantly affect the neurogenesis or maintenance of NPCs $(\mathbf{K}, \mathbf{L})$. By contrast, sustained Ascl1 expression for $72 \mathrm{hr}$ significantly induced premature neuronal formation (M-P'’). Light intensity:,$+ 0.16 \mu \mathrm{mol} / \mathrm{m}^{2} / \mathrm{s} ;++$, $1.11 \mu \mathrm{mol} / \mathrm{m}^{2} / \mathrm{s}$. VZ, Ventricular zone; SVZ, Subventricular zone; IZ, Intermediate zone; $\mathrm{CP}$, Cortical plate. ${ }^{*} p<0.05,{ }^{*} p<0.01,{ }^{* * *} p<0.001$; two-tailed Student's $t$-test. 


\begin{tabular}{|c|c|c|c|c|c|c|c|}
\hline & Strain name & $\begin{array}{c}\text { Transgenic } \\
\text { Type }\end{array}$ & $\begin{array}{l}\text { Promoter/ } \\
\text { Enhancer }\end{array}$ & $\begin{array}{c}\text { Expressed } \\
\text { protein }\end{array}$ & $\begin{array}{c}\operatorname{Poly}(A) \\
\text { signal }\end{array}$ & Reference & $\begin{array}{c}\text { RIKEN } \\
\text { BRC stock } \\
\text { number }\end{array}$ \\
\hline 1 & $\begin{array}{c}\text { pHes5-NLS-Ub- } \\
\text { luc2 } \\
\end{array}$ & $\mathrm{Tg}$ & $\begin{array}{c}\text { 3.0-kb Hes5 } \\
\text { promoter }\end{array}$ & $\begin{array}{l}\text { Ub-NLS- } \\
\text { Luc2 }\end{array}$ & $\begin{array}{c}\text { Hes5 } \\
\text { 3'-UTR }\end{array}$ & This study & $\begin{array}{l}\text { RBRC } \\
06025 \\
\end{array}$ \\
\hline 2 & $\begin{array}{c}\text { Ascl1-NLS-Ub- } \\
\text { luc2 }\end{array}$ & $\mathrm{BAC} \mathrm{Tg}$ & $\begin{array}{c}\text { Ascl1 (RP24- } \\
\text { 130P7) }\end{array}$ & $\begin{array}{c}\text { Ub-NLS- } \\
\text { Luc2 }\end{array}$ & $\begin{array}{c}\text { Ascl1 } \\
\text { 3'-UTR }\end{array}$ & This study & $\begin{array}{l}\text { RBRC } \\
06014 \\
\end{array}$ \\
\hline 3 & $\begin{array}{c}\text { Luc2-Hes1 } \\
\text { fusion }\end{array}$ & $\mathrm{BAC} \mathrm{Tg}$ & $\begin{array}{c}\text { Hes1 (RP23- } \\
8 \mathrm{~N} 20)\end{array}$ & $\begin{array}{c}\text { HA-Luc2- } \\
\text { Hes1 }\end{array}$ & $\begin{array}{c}\text { Hes1 } \\
\text { 3'-UTR }\end{array}$ & This study & $\begin{array}{l}\text { RBRC } \\
06013 \\
\end{array}$ \\
\hline 4 & $\begin{array}{c}\text { Venus-Hes1 } \\
\text { fusion }\end{array}$ & $\mathrm{KI}$ & Hes1 & Venus-Hes 1 & $\begin{array}{c}\text { Hes1 } \\
\text { 3'-UTR }\end{array}$ & $\begin{array}{c}\text { This study } \\
\&(52)\end{array}$ & $\begin{array}{l}\text { RBRC } \\
06010 \\
\end{array}$ \\
\hline 5 & $\begin{array}{l}\text { Eluc-Hes5 } \\
\text { fusion }\end{array}$ & $\mathrm{KI}$ & Hes5 & $\begin{array}{c}\text { Flag-Eluc- } \\
\text { Hes5 }\end{array}$ & $\begin{array}{c}\text { Hes5 } \\
\text { 3'-UTR }\end{array}$ & This study & $\begin{array}{l}\text { RBRC } \\
06009 \\
\end{array}$ \\
\hline 6 & $\begin{array}{l}\text { Venus-Hes5 } \\
\text { fusion }\end{array}$ & $\mathrm{KI}$ & Hes5 & $\begin{array}{c}\text { Flag-Venus- } \\
\text { Hes5 }\end{array}$ & $\begin{array}{c}\text { Hes5 } \\
\text { 3'-UTR }\end{array}$ & This study & $\begin{array}{l}\text { RBRC } \\
06008\end{array}$ \\
\hline 7 & $\begin{array}{l}\text { Luc2-Ascl1 } \\
\text { fusion }\end{array}$ & $\mathrm{BAC} \mathrm{Tg}$ & $\begin{array}{c}\text { Ascl1 (RP24- } \\
\text { 130P7) }\end{array}$ & $\begin{array}{c}\text { HA-Luc2- } \\
\text { Ascl1 }\end{array}$ & $\begin{array}{c}\text { Ascl1 } \\
\text { 3'-UTR }\end{array}$ & This study & $\begin{array}{l}\text { RBRC } \\
06019 \\
\end{array}$ \\
\hline 8 & $\begin{array}{l}\text { Venus-Ascl1 } \\
\text { fusion }\end{array}$ & BAC Tg & $\begin{array}{c}\text { Ascl1 (RP24- } \\
\text { 130P7) }\end{array}$ & $\begin{array}{l}\text { Flag-Venus- } \\
\text { Ascl1 }\end{array}$ & $\begin{array}{c}\text { Ascl1 } \\
\text { 3'-UTR }\end{array}$ & This study & $\begin{array}{l}\text { RBRC } \\
06020\end{array}$ \\
\hline 9 & $\begin{array}{l}\text { Luc2-Olig2 } \\
\text { fusion }\end{array}$ & BAC Tg & $\begin{array}{c}\text { Olig2 (RP23- } \\
\text { 356P18) }\end{array}$ & $\begin{array}{c}\text { HA-Luc2- } \\
\text { Olig2 } \\
\end{array}$ & $\begin{array}{c}\text { Olig2 } \\
\text { 3'-UTR }\end{array}$ & This study & $\begin{array}{l}\text { RBRC } \\
06022 \\
\end{array}$ \\
\hline 10 & $\begin{array}{c}\text { Venus-Olig2 } \\
\text { fusion }\end{array}$ & $\mathrm{BAC} \mathrm{Tg}$ & $\begin{array}{c}\text { Olig2 (RP23- } \\
\text { 356P18) }\end{array}$ & $\begin{array}{l}\text { Flag-Venus- } \\
\text { Olig2 }\end{array}$ & $\begin{array}{c}\text { Olig2 } \\
\text { 3'-UTR }\end{array}$ & This study & $\begin{array}{l}\text { RBRC } \\
06017 \\
\end{array}$ \\
\hline 11 & $\begin{array}{c}\text { mCherry-Olig2 } \\
\text { fusion }\end{array}$ & $\mathrm{BAC} \mathrm{Tg}$ & $\begin{array}{c}\text { Olig2 (RP23- } \\
\text { 356P18) } \\
\end{array}$ & $\begin{array}{c}\text { mCherry- } \\
\text { Olig2 }\end{array}$ & $\begin{array}{c}\text { Olig2 } \\
\text { 3'-UTR }\end{array}$ & This study & $\begin{array}{l}\text { RBRC } \\
06018 \\
\end{array}$ \\
\hline 12 & $\begin{array}{c}\text { Luc2-Sox2 } \\
\text { fusion }\end{array}$ & BAC Tg & $\begin{array}{c}\text { Sox2 (RP23- } \\
\text { 4D1) }\end{array}$ & $\begin{array}{l}\text { HA-Luc2- } \\
\text { Sox2 }\end{array}$ & $\begin{array}{c}\text { Sox2 } \\
\text { 3'-UTR }\end{array}$ & This study & $\begin{array}{l}\text { RBRC } \\
06023\end{array}$ \\
\hline 13 & DCX-DsRed & $\mathrm{Tg}$ & $\begin{array}{c}\text { 3.7-kb DCX } \\
\text { promoter }\end{array}$ & $\begin{array}{c}\text { DsRed } \\
\text { Express }\end{array}$ & SV40 & $(41)$ & \\
\hline 14 & pCNP-Venus & $\mathrm{Tg}$ & $\begin{array}{c}\text { 3.9-kb CNPase } \\
\text { promoter }\end{array}$ & $\begin{array}{l}\text { gapVenus- } \\
\text { T2A-Venus }\end{array}$ & SV40 & This study & $\begin{array}{l}\text { RBRC } \\
06021 \\
\end{array}$ \\
\hline 15 & $\begin{array}{c}\text { pCAG-mAG- } \\
\text { hGem } \\
(1 / 110) \# 504\end{array}$ & $\mathrm{Tg}$ & CAG promoter & $\begin{array}{c}\text { mAG-hGem } \\
(1 / 110)\end{array}$ & $\begin{array}{l}\text { Rabbit } \\
\beta \text {-globin }\end{array}$ & (42) & $\begin{array}{l}\text { RBRC } \\
02892\end{array}$ \\
\hline 16 & $\begin{array}{c}\text { pCAG-mKO2- } \\
\text { hCdt1(30/120) } \\
\# 596\end{array}$ & $\mathrm{Tg}$ & CAG promoter & $\begin{array}{c}\text { mKO2- } \\
\text { hCdt1 } \\
(30 / 120) \\
\end{array}$ & $\begin{array}{l}\text { Rabbit } \\
\beta \text {-globin }\end{array}$ & $(42)$ & $\begin{array}{l}\text { RBRC } \\
02892\end{array}$ \\
\hline 17 & $\begin{array}{l}\text { Rosa26-LoxP- } \\
\text { Stop- } \\
\text { LoxP-NICD- } \\
\text { iresEGFPnls }\end{array}$ & $\mathrm{KI}$ & $\begin{array}{c}\text { Rosa26 } \\
\text { promoter }\end{array}$ & $\begin{array}{l}\text { NICD \& } \\
\text { nlsEGFP }\end{array}$ & $\begin{array}{c}\text { Human } \\
\text { growth } \\
\text { hormone }\end{array}$ & $(40)$ & \\
\hline 18 & Hes1-KO & $\mathrm{KO}$ & & & & (53) & $\begin{array}{l}\text { RBRC } \\
05979 \\
\end{array}$ \\
\hline 19 & Ascl1-KO & $\mathrm{KO}$ & & & & $(54)$ & \\
\hline 20 & Olig2-KO & $\mathrm{KO}$ & & & & $(55)$ & \\
\hline
\end{tabular}

Table S1.

Genetically modified mouse strains used in this study. KO, knock-out; KI, knock-in. 


\section{Captions for Movies}

\section{Movie S1}

Luc2-Hes1 expression in slice culture of the ventral telencephalon. Time in hr and min is indicated. Related to Fig. 1.

\section{Movie S2}

Luc2-Hes1 expression in self-renewing NPCs. Time in $\mathrm{hr}$ and min is indicated. Related to Fig. 2 and fig. S8.

\section{Movie S3}

Luc2-Ascl1 expression in self-renewing NPCs. Time in $\mathrm{hr}$ and min is indicated. Related to Fig. 2 and fig. S11.

\section{Movie S4}

Luc2-Olig2 expression in self-renewing NPCs. Time in hr and min is indicated. Related to Fig. 2 and fig. S12.

\section{Movie S5}

Luc2-Sox2 expression in self-renewing NPCs. Time in hr and min is indicated. Related to fig. S14.

\section{Movie S6}

Light-induced oscillatory/sustained gene expression in NPCs. Time in hr and min is indicated. Related to Fig. 5.

\section{References}

1. H.H. Chang, M. Hemberg, M. Barahona, D.E. Ingber, S. Huang, Transcriptome-wide noise controls lineage choice in mammalian progenitor cells. Nature 453, 544-547 (2008).

2. C. Pina et al., Inferring rules of lineage commitment in haematopoiesis. Nat. Cell Biol. 14, 287-294 (2012).

3. N. Bertrand, D.S. Castro, F. Guillemot, Proneural genes and the specification of neural cell types. Nat. Rev. Neurosci. 3, 517-530 (2002).

4. S.E. Ross, M.E. Greenberg, C.D. Stiles, Basic helix-loop-helix factors in cortical development, Neuron 39, 13-25 (2003).

5. K. Tomita, K. Moriyoshi, S. Nakanishi, F. Guillemot, R. Kageyama, Mammalian achaete-scute and atonal homologs regulate neuronal versus glial fate determination in the central nervous system. EMBO J. 19, 5460-5472 (2000).

6. Y. Sun et al., Neurogenin promotes neurogenesis and inhibits glial differentiation by independent mechanisms. Cell 104, 365-376 (2001).

7. N. Nieto, S. Schuurmans, O. Britz, F. Guillemot, Neural bHLH genes control the neuronal versus glial fate decision in cortical progenitors. Neuron 29, 401-413 (2001).

8. Q.R. Lu et al., Rowitch, Common developmental requirement for Olig function indicates a motor neuron/oligodendrocyte connection. Cell 109, 75-86 (2002).

9. Q. Zhou, D.J. Anderson, The bHLH transcription factors OLIG2 and OLIG1 couple neuronal and glial subtype specification. Cell 109, 61-73 (2002).

10. H. Takebayashi et al., The basic helix-loop-helix factor Olig2 is essential for the 
development of motoneuron and oligodendrocyte lineages. Curr. Biol. 12, 1157-1163 (2002).

11. T. Ohtsuka et al., Hesl and Hes5 as Notch effectors in mammalian neuronal differentiation. EMBO J. 18, 2196-2207 (1999).

12. Y. Nakamura et al., The bHLH gene Hesl as a repressor of the neuronal commitment of CNS stem cells. J. Neurosci. 20, 283-293 (2000).

13. R. Mizuguchi et al., Combinatorial roles of olig2 and neurogenin2 in the coordinated induction of pan-neuronal and subtype-specific properties of motoneurons. Neuron 31, 757-771 (2001).

14. C.M. Parras et al., Mash1 specifies neurons and oligodendrocytes in the postnatal brain. EMBO J. 23, 4495-4505 (2004).

15. S. Gokhan et al., Combinatorial profiles of oligodendrocyte-selective classes of transcriptional regulators differentially modulate myelin basic protein gene expression. J. Neurosci. 25, 8311-8321 (2005).

16. C.M. Parras et al., The proneural gene Mash1 specifies an early population of telencephalic oligodendrocytes. J Neurosci. 27, 4233-4242 (2007).

17. E.J. Kim, J. Battsite, Y. Nakagawa, J.E. Johnson, Ascl1 (Mash1) lineage cells contribute to discrete cell populations in CNS architecture. Mol. Cell. Neurosci. 38, 595-606 (2008).

18. M.A. Petryniak, G.B. Potter, D.H. Rowitch, J.L. Rubenstein, Dlxl and Dlx2 control neuronal versus oligodendroglial cell fate acquisition in the developing forebrain. Neuron 55, 417-433 (2007).

19. E. Pastrana, L.-C. Cheng, F. Doetsch, Simultaneous prospective purification of adult subventricular zone neural stem cells and their progeny. Proc. Natl. Acad. Sci. USA 106, 6387-6392 (2009).

20. D.S. Castro et al., A novel function of the proneural factor Ascl1 in progenitor proliferation identified by genome-wide characterization of its targets. Genes Dev. 25, 930-945 (2011).

21. T. Ohtsuka, M. Sakamoto, F. Guillemot, R. Kageyama, Roles of Notch signaling in expansion of neural stem cells of the developing brain. J. Biol. Chem. 276, 3046730474 (2001).

22. Y. Wu, E.M. Levine M.S. Rao, Hes1 but not Hes5 regulates an astrocyte versus oligodendrocyte fate choice in glial restricted precursors. Dev Dyn, 226, 675-689 (2003).

23. K.L. Ligon et al., Olig2-regulated lineage-restricted pathway controls replication competence in neural stem cells and malignant glioma. Neuron 53, 503-517 (2007).

24. H. Hirata et al., Oscillatory expression of the bHLH factor Hes1 regulated by a negative feedback loop. Science 298, 840-843 (2002).

25. H. Shimojo, T. Ohtsuka, R. Kageyama, Oscillations in Notch signaling regulate maintenance of neural progenitors. Neuron 58, 52-64 (2008).

26. R. Lu et al., Systems-level dynamic analyses of fate change in murine embryonic stem cells. Nature 462, 358-362 (2009).

27. S.W. Levison, J.E. Goldman, Multipotential and lineage restricted precursors coexist in the mammalian perinatal subventricular zone. J. Neurosci. Res. 48, 83-94 (1997).

28. C.A.G. Marshall, J.E. Goldman, Subpallial Dlx2-expressing cells give rise to astrocytes and oligodendrocytes in the cerebral cortex and white matter. J. Neurosci. 22, 9821-9830 (2002).

29. S. Fukuda, T. Kondo, H. Takebayashi, T. Taga, Negative regulatory effect of an oligodendrocytic bHLH factor OLIG2 on the astrocytic differentiation pathway. Cell Death Differ. 11, 196-202 (2004).

30. L. Conti et al., A. Smith, Niche-independent symmetrical self-renewal of a mammalian tissue stem cell. PLoS Biol. 3, e283 (2005).

31. S.M. Pollard, L. Conti, Y. Sun, D. Goffredo, A. Smith, Adherent neural stem (NS) cells from fetal and adult forebrain. Cereb. Cortex 16, i112-i120 (2006). 
32. L.H. Pevny, S.K. Nicolis, Sox2 roles in neural stem cells. Int. J. Biochem. Cell Biol. 42, 421-424 (2010).

33. J.H. Baek., J. Hatakeyama, S. Sakamoto, T. Ohtsuka, R. Kageyama, Persistent and high levels of Hes 1 expression regulate boundary formation in the developing central nervous system. Development 133, 2467-2476 (2006).

34. H. Chen et al., Conservation of the Drosophila lateral inhibition pathway in human lung cancer: a hairy-related protein (HES-1) directly represses achaete-scute homolog1 expression. Proc. Natl. Acad. Sci. USA 94, 5355-5360 (1997).

35. E.H. Schroeter, J.A. Kisslinger, R. Kopan, Notch-1 signalling requires ligand-induced proteolytic release of intracellular domain. Nature 393, 382-386 (1998).

36. X. Wang, X. Chen, Y. Yang, Spatiotemporal control of gene expression by a lightswitchable transgene system. Nat. Meth. 9, 266-269 (2012).

37. C. Hindley et al., Post-translational modification of Ngn2 differentially affects transcription of distinct targets to regulate the balance between progenitor maintenance and differentiation. Development 139, 1718-1723 (2012).

38. Y. Masamizu et al., Real-time imaging of the somite segmentation clock: revelation of unstable oscillators in the individual presomitic mesoderm cell. Proc. Natl. Acad. Sci. USA 103, 1313-1318 (2006).

39. I. Imayoshi, H. Shimojo, M. Sakamoto, T. Ohtsuka, R. Kageyama, Genetic visualization of notch signaling in mammalian neurogenesis. Cell Mol Life Sci. 70, 2045-2057 (2012).

40. L.C. Murtaugh, B.Z. Stanger, K.M. Kwan, D.A. Melton, Notch signaling controls multiple steps of pancreatic differentiation. Proc. Natl. Acad. Sci. USA 100, 1492014925 (2003).

41. X. Wang, R. Qiu, W. Tsark, Q. Lu, Rapid promoter analysis in developing mouse brain and genetic labeling of young neurons by doublecortin-DsRed-express. $J$ Neurosci Res. 85, 3567-3573 (2007).

42. A. Sakaue-Sawano et al., Visualizing spatiotemporal dynamics of multicellular cellcycle progression. Cell 132, 487-498 (2008).

43. E.C. Lee et al., A highly efficient Escherichia coli-based chromosome engineering system adapted for recombinogenic targeting and subcloning of BAC DNA. Genomics 73, 56-65 (2001).

44. S. Warming et al., Simple and highly efficient BAC recombineering using galK selection. Nucleic Acids Res. 24, e36.

45. H. Miyoshi, Gene delivery to hematopoietic stem cells using lentiviral vectors. Methods Mol. Biol. 246, 429-438 (2004).

46. A. Tokunaga et al., Mapping spatio-temporal activation of Notch signaling during neurogenesis and gliogenesis in the developing mouse brain. J Neurochem. 90, 142154 (2004).

47. I. Imayoshi, T. Shimogori, T. Ohtsuka, R. Kageyama, Hes genes and neurogenin regulate non-neural versus neural fate specification in the dorsal telencephalic midline. Development 135, 2531-2541 (2008).

48. F. Doetsch, I. Caillé, D.A. Lim, J.M. García-Verdugo, A. Alvarez-Buylla, Subventricular zone astrocytes are neural stem cells in the adult mammalian brain. Cell 97, 703-716 (1999).

49. T. Miyata, A. Kawaguchi, H. Okano, M. Ogawa, Asymmetric inheritance of radial glial fibers by cortical neurons. Neuron 31, 727-741 (2001).

50 . H. Hirata et al., Instability of Hes7 protein is crucial for the somite segmentation clock. Nat. Genet. 36, 750-754 (2004).

51. M. Lema, D. Golombek, J. Echave, Delay model of the circadian pacemaker. J. Theor. Biol. 204, 565-573 (2000).

52. T. Kobayashi et al., The cyclic gene Hes1 contributes to diverse differentiation responses of embryonic stem cells. Genes Dev. 23, 1870-1875 (2009).

53. M. Ishibashi et al., Targeted disruption of mammalian hairy and Enhancer of split 
homolog-1 (HES-1) leads to up-regulation of neural helix-loop-helix factors, premature neurogenesis, and severe neural tube defects. Genes Dev. 9, 3136-3148 (1995).

54. F. Guillemot et al., Mammalian achaete-scute homolog 1 is required for the early development of olfactory and autonomic neurons. Cell 75, 463-476 (1993).

55. H. Takebayashi et al., The basic helix-loop-helix factor olig2 is essential for the development of motoneuron and oligodendrocyte lineages. Curr Biol. 12, 1157-1163 (2002). 